\title{
On the Capacity of MIMO Optical Wireless Channels
}

\author{
Longguang Li, Stefan M. Moser, Ligong Wang, Michèle Wigger*
}

20 January 2020

\begin{abstract}
This paper studies the capacity of a general multiple-input multiple-output (MIMO) free-space optical intensity channel under a per-input-antenna peak-power constraint and a total average-power constraint over all input antennas. The focus is on the scenario with more transmit than receive antennas. In this scenario, different input vectors can yield identical distributions at the output, when they result in the same image vector under multiplication by the channel matrix. We first determine the most energy-efficient input vectors that attain each of these image vectors. Based on this, we derive an equivalent capacity expression in terms of the image vector, and establish new lower and upper bounds on the capacity of this channel. The bounds match when the signal-to-noise ratio (SNR) tends to infinity, establishing the high-SNR asymptotic capacity. We also characterize the low-SNR slope of the capacity of this channel.
\end{abstract}

Index terms - Average- and peak-power constraints, channel capacity, direct detection, Gaussian noise, infrared communication, multiple-input multiple-output (MIMO) channel, optical communication.

\section{Introduction}

This paper considers an optical wireless communication system where the transmitter modulates the intensity of optical signals coming from light emitting diodes (LEDs) or laser diodes (LDs), and the receiver measures incoming optical intensities by means of photodetectors [1]-[3]. Such intensity-modulation-direct-detection (IM-DD) systems are appealing because of their simplicity and their good performance at relatively low costs. As a first approximation, the noise in such systems can be assumed to be Gaussian and independent of the transmitted signal [4, Ch. 1, p. 3], [5], [6]. Inputs are nonnegative and typically subject to both peak- and average-power constraints, where the peak-power constraint is mainly due to technical limitations of the used components and where the average-power constraint is imposed by battery limitations and safety considerations. We should notice that, unlike in radio-frequency communication, the average-power constraint applies directly to the transmitted signal and not to its square, because the power of the transmitted signal is proportional to the optical intensity and hence relates directly to the transmitted signal.

IM-DD systems have been extensively studied in recent years [5], [7]-[20], with an increasing interest in multiple-input multiple-output (MIMO) systems where transmitters are equipped with $n_{\mathrm{T}}>1$ LEDs or LDs and receivers with $n_{\mathrm{R}}>1$ photodetectors.

* L. Li was with LTCI, Telecom ParisTech, Université Paris-Saclay, 75013 Paris, France, and is now with the Department of Electrical and Computer Engineering at National University of Singapore, Singapore. S. Moser is with the Signal and Information Processing Lab, ETH Zurich, Switzerland and with the Institute of Communications Engineering at National Chiao Tung University, Hsinchu, Taiwan. L. Wang is with ETIS-Université Paris Seine, Université de (NUS Cergy-Pontoise, ENSEA, CNRS, Cergy-Pontoise, France. M. Wigger is with LTCI, Telecom ParisTech, Université Paris-Saclay, 75013 Paris, France. This work was presented in part at the IEEE Information Theory Workshop, Guangzhou, China, November 2018. 
Practical transmission schemes for such systems with different modulation methods, such as pulse-position modulation or LED index modulation based on orthogonal frequencydivision multiplexing, were presented in [21]-[23]. Code constructions were described in $[24]-[26]$.

A previous work [16] presented upper and lower bounds on the capacity when the channel matrix is of full column-rank (so necessarily $n_{\mathrm{R}} \geq n_{\mathrm{T}}$ ) and determined the asymptotic capacity at high signal-to-noise ratio (SNR) exactly. For general MIMO channels with average-power constraints only, the asymptotic high-SNR capacity was determined in [17], [18]. The works [17], [18] also study general MIMO channels with both peak- and average-power constraints, but they only determine the high-SNR pre-log (degrees of freedom), and not the exact asymptotic capacity. The work in [19] considers general MIMO channels but with peak-power constraints only.

The works most related to ours are [14], [15], [20]. For the MISO case, [14], [15] show that the optimal signaling strategy is to rely as much as possible on antennas with larger channel gains. Specifically, if an antenna is used for active signaling in a channel use, then all antennas with larger channel gains should transmit at maximum allowed peak power $A$, and all antennas with smaller channel gains should be silenced, i.e., send 0 . It is shown that this antenna-cooperation strategy is optimal at all SNRs.

In [20], the asymptotic capacity in the low-SNR regime is considered for general MIMO channels under both a peak- and an average-power constraint. It is shown that the asymptotically optimal input distribution in the low-SNR regime puts the antennas into a certain order and assigns positive mass points only to input vectors in $\{0, A\}^{n_{\mathrm{T}}}$ in such a way that, if a given input antenna is set to full power $A$, then also all preceding antennas in the specified order are set to $A$. This strategy is reminiscent of the optimal signaling strategy for MISO channels [14], [15]. However, whereas the optimal order in [20] needs to be determined numerically, in the MISO case the optimal order naturally follows the channel strengths of the input antennas. Furthermore, the order in [14], [15] is optimal at all SNRs, whereas the order in [20] is shown to be optimal only in the asymptotic low-SNR limit.

The current paper focuses on MIMO channels with more transmit than receive antennas, i.e., more LEDs than photodetectors:

$$
n_{\mathrm{T}}>n_{\mathrm{R}}>1
$$

Such a system arises for example when the transmitter is based on an existing illumination system consisting of a large number of LEDs, whereas the receiver photodetectors are purchased at additional cost.

Our main contributions are as follows:

1. Minimum-Energy Signaling: The optimal signaling strategy for MISO channels of [14], [15] is generalized to MIMO channels with $n_{\mathrm{T}}>n_{\mathrm{R}}>1$. For each "image vector" $\overline{\mathbf{x}}$ - an $n_{\mathrm{R}}$-dimensional vector that can be produced by multiplying an input vector $\mathbf{x}$ by the channel matrix - Lemma 5 identifies the input vector $\mathbf{x}_{\text {min }}$ that induces $\overline{\mathbf{x}}$ with minimum total energy. The minimum-energy signaling strategy partitions the image space of vectors $\overline{\mathbf{x}}$ into at most $\left(\begin{array}{l}n_{\mathrm{T}} \\ n_{\mathrm{R}}\end{array}\right)$ parallelepipeds, each one spanned by a different subset of $n_{\mathrm{R}}$ linearly independent columns of the channel matrix (see Figures 1-5). In each parallelepiped, the minimum-energy signaling sets the $n_{\mathrm{T}}-n_{\mathrm{R}}$ inputs corresponding to the columns that were not chosen either to 0 or to $A$ according to a predescribed rule and uses the $n_{\mathrm{R}}$ inputs corresponding to the chosen columns for signaling within the parallelepiped.

2. Equivalent Capacity Expression: Using Lemma 5, Proposition 8 expresses the capacity of the MIMO channel in terms of the random image vector $\overline{\mathbf{X}}$. In particular, the power constraints on the input vector are translated into a set of constraints on $\overline{\mathbf{X}}$. 
3. Maximizing the Trace of the Covariance Matrix: The low-SNR slope of the capacity of the MIMO channel is determined by the maximum trace of the covariance matrix of $\overline{\mathbf{X}}$ [20]. Lemmas 10, 11, and 12 establish several properties for the optimal input distribution that maximizes this trace. They restate the result in [20] that the covariance-trace maximizing input distribution puts positive mass points only on $\{0, A\}^{n_{\mathrm{T}}}$ in a way that if an antenna is set to $A$, then all preceding antennas in a specified order are also set to $A$. The lemmas restrict the search space for finding the optimal antenna ordering and show that the optimal probability mass function (PMF) puts nonzero probability to the origin and to at most $n_{\mathrm{R}}+1$ other input vectors.

4. Lower Bounds: Lower bounds on the capacity of the channel of interest are obtained by applying the Entropy Power Inequality (EPI) [27] and choosing input vectors that maximize the differential entropy of $\overline{\mathbf{X}}$ under the imposed power constraints; see Theorems 15 and 16.

5. Upper Bounds: Three capacity upper bounds are derived by means of the equivalent capacity expression in Proposition 8 and the duality-based upper-bounding technique for capacity; see Theorems 17, 18, and 19. Another upper bound uses simple maximum-entropy arguments and algebraic manipulations; see Theorem 20.

6. Asymptotic Capacity: Theorem 21 presents the asymptotic capacity when the SNR tends to infinity, and Theorem 22 gives the slope of capacity when the SNR tends to zero. (This later result was already proven in [20], but as described above, our results simplify the computation of the slope.)

The paper is organized as follows. We end the introduction with a few notational conventions. Section 2 provides details of the investigated channel model. Section 3 identifies the minimum-energy signaling schemes. Section 4 provides an equivalent expression for the capacity of the channel. Section 5 shows properties of maximum-variance signaling schemes. Section 6 presents all new lower and upper bounds on the channel capacity, and also gives the high- and low-SNR asymptotics. The paper is concluded in Section 7. Most of the proofs are in the appendices.

Notation: We distinguish between random and deterministic quantities. A random variable is denoted by a capital Roman letter, e.g., $Z$, while its realization is denoted by the corresponding small Roman letter, e.g., z. Vectors are boldfaced, e.g., $\mathbf{X}$ denotes a random vector and $\mathbf{x}$ its realization. All the matrices in this paper are deterministic, which are denoted in capital letters, and are typeset in a sans-serif font, e.g., H. Sets are denoted by capital letters in a calligraphic font, e.g., $\mathcal{U}$ or $\mathscr{U}$ (the latter typically denoting a set of sets), except the set of real numbers that is designated by $\mathfrak{R}$. We further use another special font for random sets, e.g., $\mathbb{U}$. Constants are typeset either in small Romans, in Greek letters, or in a special font, e.g., E or A. Entropy is typeset as $\mathrm{H}(\cdot)$, differential entropy as $h(\cdot)$, and mutual information as $\mathrm{I}(\cdot ; \cdot)$. The relative entropy (Kullback-Leibler divergence) between probability vectors $\mathbf{p}$ and $\mathbf{q}$ is denoted by $\mathrm{D}(\mathbf{p} \| \mathbf{q})$. We will use the $\mathcal{L}_{1}$-norm, which we indicate by $\|\cdot\|_{1}$, while $\|\cdot\|_{2}$ denotes the $\mathcal{L}_{2}$-norm. The logarithmic function $\log (\cdot)$ denotes the natural logarithm. The Lebesgue measure of a set $\mathcal{A} \subseteq \mathfrak{R}^{n}$ is denoted by $\operatorname{vol}(\mathcal{A})$.

\section{Channel Model}

Consider an $n_{\mathrm{R}} \times n_{\mathrm{T}}$ MIMO channel

$$
\mathbf{Y}=\mathrm{Hx}+\mathbf{Z}
$$


where $\mathbf{x}=\left(x_{1}, \ldots, x_{n_{\mathrm{T}}}\right)^{\top}$ denotes the $n_{\mathrm{T}}$-dimensional real-valued channel input vector, where $\mathbf{Z}$ denotes the $n_{\mathrm{R}}$-dimensional real-valued noise vector, and where

$$
\mathrm{H}=\left[\mathbf{h}_{1}, \mathbf{h}_{2}, \ldots, \mathbf{h}_{n_{\mathrm{T}}}\right]
$$

is the deterministic real-valued $n_{\mathrm{R}} \times n_{\mathrm{T}}$ channel matrix (hence $\mathbf{h}_{1}, \ldots, \mathbf{h}_{n_{\mathrm{T}}}$ are $n_{\mathrm{R}^{-}}$ dimensional column vectors).

The channel matrix $\mathrm{H}$ models the crosstalk among different channel inputs in terms of optical intensity, and thus, in realistic situations, its components are nonnegative. However, for the purpose of generality, in this work we allow the entries of $\mathrm{H}$ to take negative values, i.e., they can be any real numbers. The additive noise $\mathbf{Z}$ describes random fluctuations caused by thermal noise and by ambient light arriving at the receiver, where we assume that the receiver has already removed its expectation of the influence of the ambient light. Since the expected intensity of ambient light is typically much larger than its fluctuations, and since the thermal noise can be negative, the additive noise $Z$ can also be negative (even when $\mathrm{H}$ only has nonnegative entries). In this paper, we assume that the noise vector is independent of the channel input $\mathbf{X}$ and that it has independent standard Gaussian entries,

$$
\mathbf{Z} \sim \mathcal{N}(0, \mathrm{I}) .
$$

The channel inputs correspond to optical intensities sent by the LEDs, hence they are nonnegative:

$$
x_{k} \in \mathfrak{R}_{0}^{+}, \quad k=1, \ldots, n_{\mathrm{T}} .
$$

We assume the inputs to be subject to a peak-power (peak-intensity) and an averagepower (average-intensity) constraint:

$$
\begin{aligned}
\operatorname{Pr}\left[X_{k}>\mathrm{A}\right] & =0, \quad \forall k \in\left\{1, \ldots, n_{\mathrm{T}}\right\}, \\
\mathrm{E}\left[\|\mathbf{X}\|_{1}\right] & \leq \mathrm{E},
\end{aligned}
$$

for some fixed parameters $A, E>0$. As mentioned in the introduction, the averagepower constraint is on the expectation of the channel input and not on its square. Also note that $A$ describes the maximum power of each single LED, while $E$ describes the allowed total average power of all LEDs together. We denote the ratio between the allowed average power and the allowed peak power by $\alpha$ :

$$
\alpha \triangleq \frac{E}{A} \text {. }
$$

Throughout this paper, we assume that

$$
n_{\mathrm{T}}>n_{\mathrm{R}} \text { and } \operatorname{rank}(\mathrm{H})=n_{\mathrm{R}}
$$

The second assumption does not incur any loss of generality. Indeed, if

$$
n_{\mathrm{T}}>n_{\mathrm{R}}>\operatorname{rank}(\mathrm{H}),
$$

then the receiver can perform the singular-value decomposition (SVD) $H=U \Sigma V^{\top}$, compute $U^{\top} \mathbf{Y}$, and then discard the entries in $U^{\top} \mathbf{Y}$ that correspond to zero singular values. Since $U$ is invertible, this new channel has the same capacity as the original channel, but the rank of the new channel matrix is equal to the (reduced) number of receive antennas. For more details, see Appendix A.

The first assumption in (8), however, is crucial to the current setting. The situation with $n_{\mathrm{T}} \leq n_{\mathrm{R}}$ and $\operatorname{rank}(\mathrm{H})=n_{\mathrm{T}}$ was studied in [16], where it is shown that, when $n_{\mathrm{T}}<n_{\mathrm{R}}$, the channel can be transformed into an equivalent $n_{\mathrm{T}} \times n_{\mathrm{T}}$ channel. More generally, one can show that any channel with

$$
\operatorname{rank}(\mathrm{H}) \triangleq r \leq n_{\mathrm{T}} \leq n_{\mathrm{R}}
$$


can be transformed into an equivalent channel of dimension $r \times n_{\mathrm{T}}$. The idea is again to use the SVD $\mathrm{H}=\mathrm{U} \Sigma \mathrm{V}^{\top}$ to transform $\mathbf{Y}$ into $\mathrm{U}^{\top} \mathbf{Y}$, and to discard the last $n_{\mathrm{R}}-r$ entries in $\mathrm{U}^{\top} \mathbf{Y}$. If $r=n_{\mathrm{T}}$, we end up with a full-rank square $n_{\mathrm{T}} \times n_{\mathrm{T}}$ channel matrix - a case that is not studied in this paper, but that it is the most widely studied MIMO setup in the literature (e.g., [16], [18]). If $r<n_{\mathrm{T}}$, we have transformed the channel into an equivalent one that satisfies our assumptions (8). For more details, we again refer to Appendix A.

As we shall see, when $n_{\mathrm{T}}>n_{\mathrm{R}}$, it is in general suboptimal to discard $n_{\mathrm{T}}-n_{\mathrm{R}}$ transmit antennas; instead, the optimal signaling scheme involves collaboration of all transmit antennas.

In this paper we are interested in deriving capacity bounds for a channel satisfying (8). The capacity has the standard formula

$$
\mathrm{C}_{\mathbf{H}}(\mathrm{A}, \alpha A)=\max _{P_{\mathbf{X}} \text { satisfying }(6)} \mathrm{I}(\mathbf{X} ; \mathbf{Y}) .
$$

The next proposition shows that, when $\alpha>\frac{n_{\mathrm{T}}}{2}$, the channel essentially reduces to one with only a peak-power constraint. The other case where $\alpha \leq \frac{n_{\mathrm{T}}}{2}$ will be the main focus of this paper.

Proposition 1. If $\alpha>\frac{n_{\mathrm{T}}}{2}$, then the average-power constraint (6b) is inactive, i.e.,

$$
\mathrm{C}_{\mathrm{H}}(\mathrm{A}, \alpha \mathrm{A})=\mathrm{C}_{\mathrm{H}}\left(\mathrm{A}, \frac{n_{\mathrm{T}}}{2} \mathrm{~A}\right), \quad \alpha>\frac{n_{\mathrm{T}}}{2} .
$$

If $\alpha \leq \frac{n_{\mathrm{T}}}{2}$, then there exists a capacity-achieving input distribution $P_{\mathbf{X}}$ in (11) that satisfies the average-power constraint (6b) with equality.

Proof: See Appendix B.

We can alternatively write the MIMO channel as

$$
\mathbf{Y}=\overline{\mathbf{x}}+\mathbf{Z}
$$

where we set

$$
\overline{\mathbf{x}} \triangleq \mathrm{Hx}
$$

We introduce the following notation. For a matrix $\mathrm{M}=\left[\mathbf{m}_{1}, \ldots, \mathbf{m}_{k}\right]$, where $\left\{\mathbf{m}_{i}\right\}$ are column vectors, define the set

$$
\mathcal{R}(\mathrm{M}) \triangleq\left\{\sum_{i=1}^{k} \lambda_{i} \mathbf{m}_{i}: \lambda_{1}, \ldots, \lambda_{k} \in[0, \mathrm{~A}]\right\} .
$$

Note that this set is a zonotope. Since the $n_{\mathrm{T}}$-dimensional input vector $\mathbf{x}$ is constrained to the $n_{\mathrm{T}}$-dimensional hypercube $[0, \mathrm{~A}]^{n_{\mathrm{T}}}$, the $n_{\mathrm{R}}$-dimensional image vector $\overline{\mathbf{x}}$ takes value in the zonotope $\mathcal{R}(\mathrm{H})$.

For each $\overline{\mathbf{x}} \in \mathcal{R}(\mathrm{H})$, let

$$
\mathcal{S}(\overline{\mathbf{x}}) \triangleq\left\{\mathbf{x} \in[0, A]^{n_{\mathrm{T}}}: \mathrm{H} \mathbf{x}=\overline{\mathbf{x}}\right\}
$$

be the set of input vectors inducing $\overline{\mathbf{x}}$. In the following section we derive the most energy-efficient signaling method to attain a given $\overline{\mathbf{x}}$. This will allow us to express the capacity in terms of $\overline{\mathbf{X}}=\mathbf{H X}$ instead of $\mathbf{X}$, which will prove useful.

\section{Minimum-Energy Signaling}

The goal of this section is to identify for every $\overline{\mathbf{x}} \in \mathcal{R}(\mathrm{H})$ the minimum-energy choice of input vector $\mathbf{x}$ that induces $\overline{\mathbf{x}}$. Since the energy of an input vector $\mathbf{x}$ is $\|\mathbf{x}\|_{1}$, we are interested in finding an $\mathbf{x}_{\min }$ that satisfies

$$
\left\|\mathbf{x}_{\min }\right\|_{1}=\min _{\mathbf{x} \in \mathcal{S}(\overline{\mathbf{x}})}\|\mathbf{x}\|_{1}
$$


We start by describing (without proof) the choice of $\mathbf{x}_{\min }$ in two different $2 \times 3$ examples.

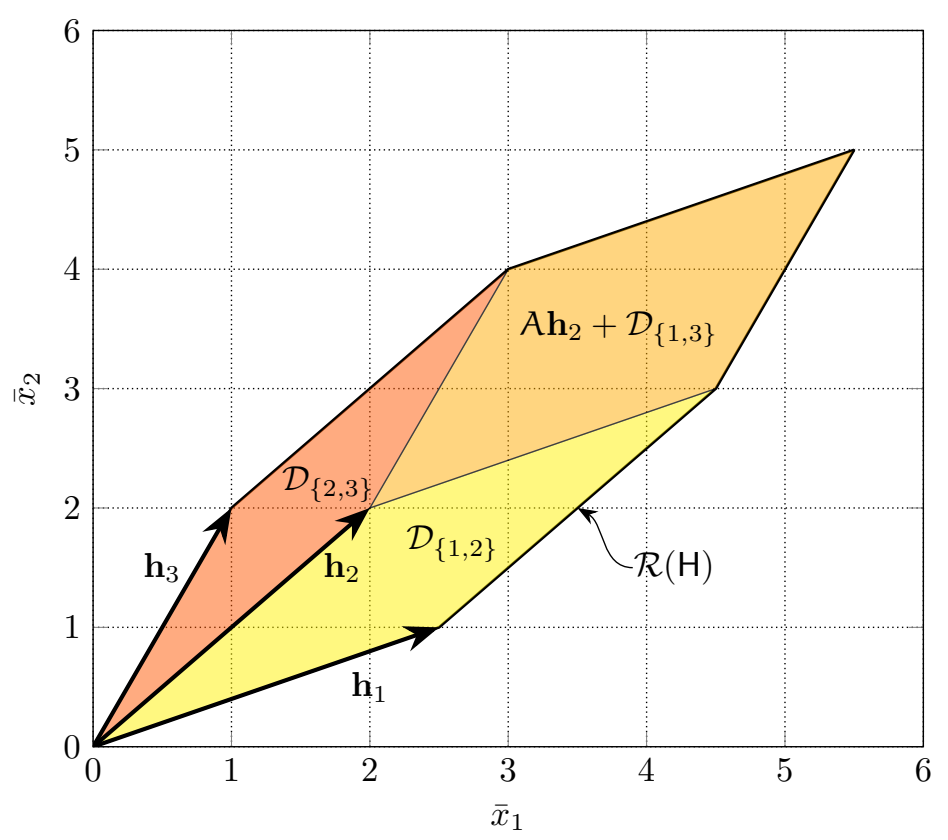

Figure 1: The zonotope $\mathcal{R}(\mathrm{H})$ for the $2 \times 3$ MIMO channel matrix $\mathrm{H}=[2.5,2,1 ; 1,2,2]$ given in (18) and its minimum-energy decomposition into three parallelograms. The peak power is assumed to be $A=1$.

Example 2. Consider the $2 \times 3$ MIMO channel matrix

$$
\mathrm{H}=\left(\begin{array}{ccc}
2.5 & 2 & 1 \\
1 & 2 & 2
\end{array}\right)
$$

composed of the three column vectors $\mathbf{h}_{1}=(2.5,1)^{\top}, \mathbf{h}_{2}=(2,2)^{\top}$, and $\mathbf{h}_{3}=(1,2)^{\top}$. Figure 1 depicts the zonotope $\mathcal{R}(\mathrm{H})$ and partitions it into three parallelograms based on three different forms of $\mathbf{x}_{\text {min }}$. For any $\overline{\mathbf{x}}$ in the parallelogram $\mathcal{D}_{\{1,2\}} \triangleq \mathcal{R}\left(\mathrm{H}_{\{1,2\}}\right)$, where $\mathrm{H}_{\{1,2\}} \triangleq\left[\mathbf{h}_{1}, \mathbf{h}_{2}\right]$, the minimum-energy input $\mathbf{x}_{\min }$ inducing $\overline{\mathbf{x}}$ has 0 as its third component. Since $\mathrm{H}_{\{1,2\}}$ has full rank, there is only one such input inducing $\overline{\mathbf{x}}$ :

$$
\mathbf{x}_{\min }=\left(\begin{array}{c}
\mathbf{H}_{\{1,2\}}^{-1} \overline{\mathbf{x}} \\
0
\end{array}\right), \quad \text { if } \overline{\mathbf{x}} \in \mathcal{D}_{\{1,2\}}
$$

Similarly, for any $\overline{\mathbf{x}}$ in the parallelogram $\mathcal{D}_{\{2,3\}} \triangleq \mathcal{R}\left(\mathrm{H}_{\{2,3\}}\right)$, where $\mathrm{H}_{\{2,3\}} \triangleq\left[\mathbf{h}_{2}, \mathbf{h}_{3}\right]$, the minimum-energy input $\mathbf{x}_{\min }$ inducing $\overline{\mathbf{x}}$ has 0 as its first component:

$$
\mathbf{x}_{\min }=\left(\begin{array}{c}
0 \\
\mathrm{H}_{\{2,3\}}^{-1} \overline{\mathbf{x}}
\end{array}\right), \quad \text { if } \overline{\mathbf{x}} \in \mathcal{D}_{\{2,3\}} .
$$

Finally, for any $\overline{\mathbf{x}}$ in the parallelogram $A \mathbf{h}_{2}+\mathcal{D}_{\{1,3\}}$, where $\mathcal{D}_{\{1,3\}} \triangleq \mathcal{R}\left(\mathrm{H}_{\{1,3\}}\right)$ and $\mathrm{H}_{\{1,3\}} \triangleq\left[\mathbf{h}_{1}, \mathbf{h}_{3}\right]$, the minimum-energy input $\mathbf{x}_{\min }$ inducing $\overline{\mathbf{x}}$ has $A$ as its second component:

$$
\mathbf{x}_{\min }=\left(\begin{array}{c}
x_{\min , 1} \\
A \\
x_{\min , 3}
\end{array}\right), \quad \text { if } \overline{\mathbf{x}} \in A \mathbf{h}_{2}+\mathcal{D}_{\{1,3\}}
$$


where

$$
\left(\begin{array}{l}
x_{\min , 1} \\
x_{\min , 3}
\end{array}\right)=\mathrm{H}_{\{1,3\}}^{-1}\left(\overline{\mathbf{x}}-\mathrm{A} \mathbf{h}_{2}\right) .
$$

These minimum-energy choices of $\mathbf{x}$ can be understood intuitively. Recall that we search for a triple $\mathbf{x}=\left(x_{1}, x_{2}, x_{3}\right) \in[0, \mathrm{~A}]^{3}$ that induces some given $\overline{\mathbf{x}} \in \mathcal{R}(\mathrm{H})$ :

$$
x_{1} \mathbf{h}_{1}+x_{2} \mathbf{h}_{2}+x_{3} \mathbf{h}_{3}=\overline{\mathbf{x}} \text {. }
$$

Our aim is to find an $\mathbf{x}$ with minimum $\mathcal{L}_{1}$ norm, i.e., $x_{1}+x_{2}+x_{3}$ shall be small. Now, we observe that in our example we can write

$$
\mathbf{h}_{2}=0.5 \mathbf{h}_{1}+0.75 \mathbf{h}_{3} .
$$

Since here the weights add up to more than one, $0.5+0.75>1$, it is energy-efficient to reduce $x_{1}$ and $x_{3}$ at the cost of increasing $x_{2}$. So, whenever possible, we reduce one of $x_{1}$ and $x_{3}$ to zero. This covers $\mathcal{D}_{\{1,2\}}$ and $\mathcal{D}_{\{2,3\}}$. The third parallelogram $A \mathbf{h}_{2}+\mathcal{D}_{\{1,3\}}$ can only be reached if all three components are nonzero. In this case, it is best to set the efficient component $x_{2}$ to its maximal value $\mathrm{A}$ and then use the other two to reach to $\overline{\mathbf{x}}$.

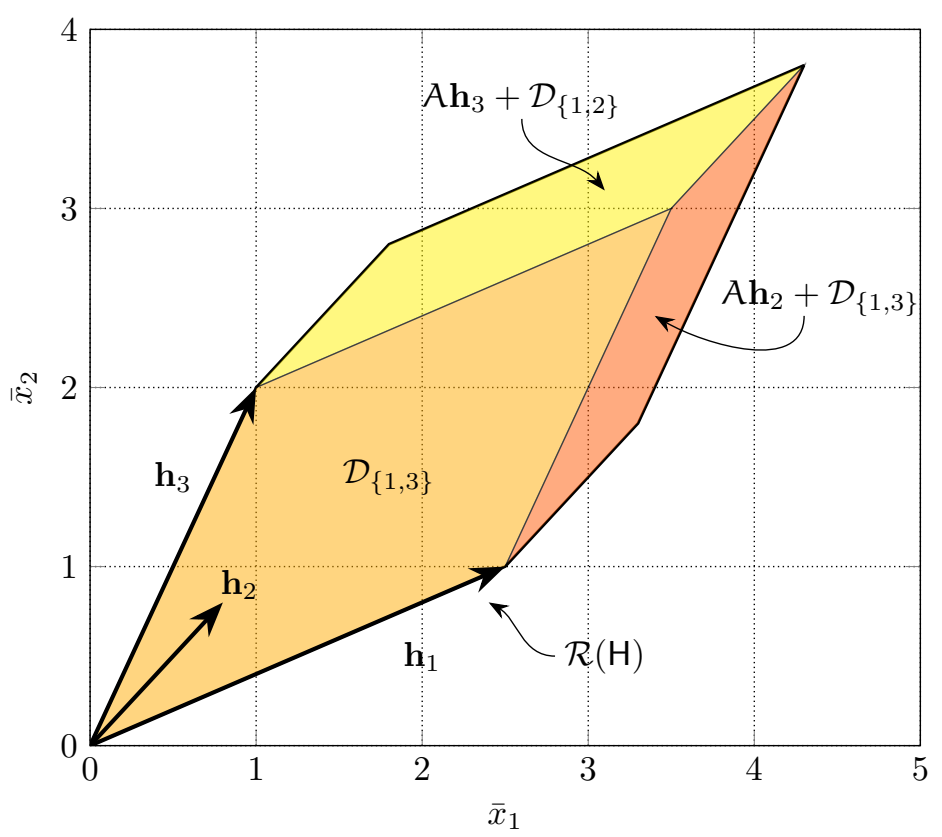

Figure 2: The zonotope $\mathcal{R}(\mathrm{H})$ for the $2 \times 3$ MIMO channel matrix $\mathrm{H}=[2.5,0.8,1 ; 1,0.8,2]$ given in (25) and its minimum-energy decomposition into three parallelograms. The peak power is $A=1$.

Example 3. We now consider another $2 \times 3$ MIMO channel with channel matrix

$$
\mathrm{H}=\left(\begin{array}{ccc}
2.5 & 0.8 & 1 \\
1 & 0.8 & 2
\end{array}\right) .
$$

Figure 2 depicts the zonotope $\mathcal{R}(\mathrm{H})$ and its partitioning based on three different forms of $\mathbf{x}_{\min }$.

Note that $\mathbf{h}_{1}$ and $\mathbf{h}_{3}$ are kept the same as in Example 2, but $\mathbf{h}_{2}$ is reduced in length. It can now be written as

$$
\mathbf{h}_{2}=0.2 \mathbf{h}_{1}+0.3 \mathbf{h}_{3} .
$$


Since here the sum of the weights is less than one, $0.2+0.3<1$, it is energy-efficient to reduce $x_{2}$ at the cost of increasing $x_{1}$ and $x_{3}$ in (23). Thus, whenever possible we keep $x_{2}=0$ and only use $x_{1}$ and $x_{3}$; this is how we cover $\mathcal{D}_{\{1,3\}}$. The other two parallelograms can only be reached with all three components being positive. In these cases, it is best to set one of $x_{1}$ and $x_{3}$ to its maximal value $A$ and use the other two to reach to $\overline{\mathbf{x}}$. $\diamond$

We now generalize Examples 2 and 3 to formally solve the optimization problem in (17) for an arbitrary $n_{\mathrm{R}} \times n_{\mathrm{T}}$ channel matrix $\mathrm{H}$. To this end, we need some further definitions. Denote by $\mathscr{U}$ the set of all choices of $n_{\mathrm{R}}$ columns of $\mathrm{H}$ that are linearly independent:

$$
\mathscr{U} \triangleq\left\{\mathcal{U}=\left\{i_{1}, \ldots, i_{n_{\mathrm{R}}}\right\} \subseteq\left\{1, \ldots, n_{\mathrm{T}}\right\}: \mathbf{h}_{i_{1}}, \ldots, \mathbf{h}_{i_{n_{\mathrm{R}}}} \text { are linearly independent }\right\} .
$$

For every one of these index sets $\mathcal{U} \in \mathscr{U}$, we denote its complement by

$$
\mathcal{U}^{\mathrm{c}} \triangleq\left\{1, \ldots, n_{\mathrm{T}}\right\} \backslash \mathcal{U}
$$

we define the $n_{\mathrm{R}} \times n_{\mathrm{R}}$ matrix $\mathrm{H}_{\mathcal{U}}$ containing the columns of $\mathrm{H}$ indicated by $\mathcal{U}$ :

$$
\mathrm{H}_{\mathcal{U}} \triangleq\left[\mathbf{h}_{i}: i \in \mathcal{U}\right]
$$

and we define the $n_{\mathrm{R}}$-dimensional parallelepiped

$$
\mathcal{D}_{\mathcal{U}} \triangleq \mathcal{R}\left(\mathrm{H}_{\mathcal{U}}\right)
$$

Notice that

$$
\operatorname{vol}\left(\mathcal{D}_{\mathcal{U}}\right)=A^{n}\left|\operatorname{det} \mathrm{H}_{\mathcal{U}}\right|
$$

which is positive because the columns of $\mathrm{H}_{\mathcal{U}}$ are linearly independent.

We shall see (Lemma 5 ahead) that $\mathcal{R}(\mathrm{H})$ can be partitioned into parallelepipeds that are shifted versions of $\left\{\mathcal{D}_{\mathcal{U}}\right\}$ in such a way that, within each parallelepiped, $\mathbf{x}_{\text {min }}$ has the same form, in a sense similar to (19)-(22) in Example 2. To specify our partition, we define the $n_{\mathrm{R}}$-dimensional vectors

$$
\gamma_{\mathcal{U}, j} \triangleq \mathrm{H}_{\mathcal{U}}^{-1} \mathbf{h}_{j}, \quad \mathcal{U} \in \mathscr{U}, j \in \mathcal{U}^{\mathrm{c}},
$$

and the sum of their components

$$
a_{\mathcal{U}, j} \triangleq \mathbf{1}_{n_{\mathrm{R}}}^{\top} \gamma_{\mathcal{U}, j}, \quad \mathcal{U} \in \mathscr{U}, j \in \mathcal{U}^{\mathrm{c}} .
$$

We next choose a set of coefficients $\left\{g_{\mathcal{U}, j}\right\}_{\mathcal{U} \in \mathscr{U}, j \in \mathcal{U}^{c}}$, which are either 0 or 1 , as follows.

- If

$$
a_{\mathcal{U}, j} \neq 1, \quad \forall \mathcal{U} \in \mathscr{U}, \forall j \in \mathcal{U}^{\mathrm{c}},
$$

then let

$$
g_{\mathcal{U}, j} \triangleq\left\{\begin{array}{ll}
1 & \text { if } a_{\mathcal{U}, j}>1, \\
0 & \text { otherwise, }
\end{array} \quad \mathcal{U} \in \mathscr{U}, j \in \mathcal{U}^{\mathrm{c}} .\right.
$$

- If (34) is violated, then we have one or several ties, i.e., the solution to the minimization problem in (17) is not unique and there exist several different but equivalent vectors $\mathbf{x}_{\min }$. (To give an example in the spirit of Examples 2 and 3, consider the channel matrix

$$
\mathrm{H}=\left(\begin{array}{ccc}
2.5 & 1.6 & 1 \\
1 & 1.6 & 2
\end{array}\right)
$$

for which $\mathbf{h}_{2}=0.4 \mathbf{h}_{1}+0.6 \mathbf{h}_{3}$ with $0.4+0.6=1$.)

In order to break such ties, the following algorithm simply picks one out of all possible equivalent optimal choices. 


\section{Algorithm 4.}

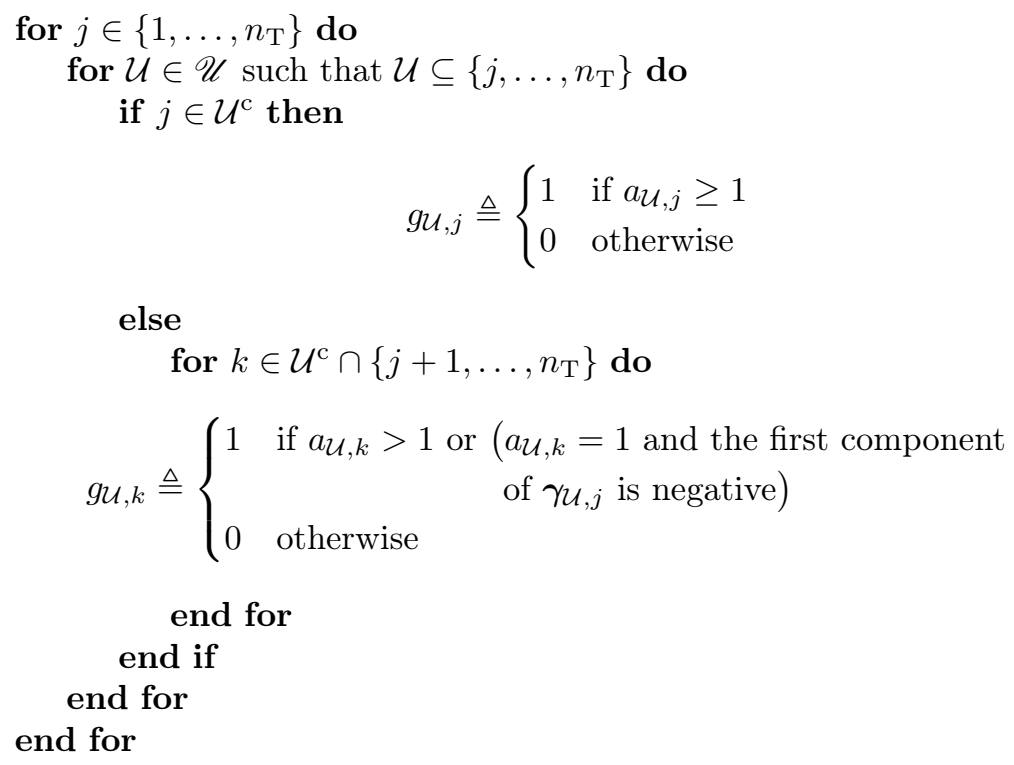

Finally, let

$$
\mathbf{v}_{\mathcal{U}} \triangleq A \sum_{j \in \mathcal{U}^{\mathrm{c}}} g_{\mathcal{U}, j} \mathbf{h}_{j}, \quad \mathcal{U} \in \mathscr{U}
$$

We are now ready to describe our partition of $\mathcal{R}(\mathrm{H})$.

Lemma 5. For each $\mathcal{U} \in \mathscr{U}$ let $\mathcal{D}_{\mathcal{U}},\left\{g_{\mathcal{U}, j}\right\}_{j \in \mathcal{U}^{c}}$, and $\mathbf{v}_{\mathcal{U}}$ be as given in (30), (35) or Algorithm 4, and (39), respectively.

1. The zonotope $\mathcal{R}(\mathrm{H})$ is covered by the parallelepipeds $\left\{\mathbf{v}_{\mathcal{U}}+\mathcal{D}_{\mathcal{U}}\right\}_{\mathcal{U} \in \mathscr{U}}$, which overlap only on sets of measure zero:

$$
\bigcup_{\mathcal{U} \in \mathscr{U}}\left(\mathbf{v}_{\mathcal{U}}+\mathcal{D}_{\mathcal{U}}\right)=\mathcal{R}(\mathrm{H})
$$

and

$$
\operatorname{vol}\left(\left(\mathbf{v}_{\mathcal{U}}+\mathcal{D}_{\mathcal{U}}\right) \cap\left(\mathbf{v}_{\mathcal{V}}+\mathcal{D}_{\mathcal{V}}\right)\right)=0, \quad \mathcal{U}, \mathcal{V} \in \mathscr{U}, \mathcal{U} \neq \mathcal{V}
$$

where we recall that $\operatorname{vol}(\cdot)$ denotes the $\left(n_{\mathrm{R}}\right.$-dimensional) Lebesgue measure.

2. Fix some $\mathcal{U} \in \mathscr{U}$ and some $\overline{\mathbf{x}} \in \mathbf{v}_{\mathcal{U}}+\mathcal{D}_{\mathcal{U}}$. A vector that induces $\overline{\mathbf{x}}$ with minimum energy, i.e., $\mathbf{x}_{\min }$ in $(17)$, is given by $\mathbf{x}=\left(x_{1}, \ldots, x_{n_{\mathrm{T}}}\right)^{\top}$, where

$$
x_{i}= \begin{cases}\mathrm{A} \cdot g_{\mathcal{U}, i} & \text { if } i \in \mathcal{U}^{\mathrm{c}}, \\ \beta_{i} & \text { if } i \in \mathcal{U},\end{cases}
$$

where the vector $\boldsymbol{\beta}=\left(\beta_{i}: i \in \mathcal{U}\right)^{\top}$ is given by

$$
\boldsymbol{\beta} \triangleq \mathrm{H}_{\mathcal{U}}^{-1}\left(\overline{\mathbf{x}}-\mathbf{v}_{\mathcal{U}}\right)
$$

Proof: See Appendix C.

We recall that Figures 1 and 2 show the partition of $\mathcal{R}(\mathrm{H})$ into the union (40) for two examples of $2 \times 3$ channel matrices. Figures 3-6 show four more examples. Among them, Figure 5 illustrates a case where $\mathrm{H}$ contains linearly dependent columns, and Figure 6 shows a case where $\mathrm{H}$ has negative entries. 


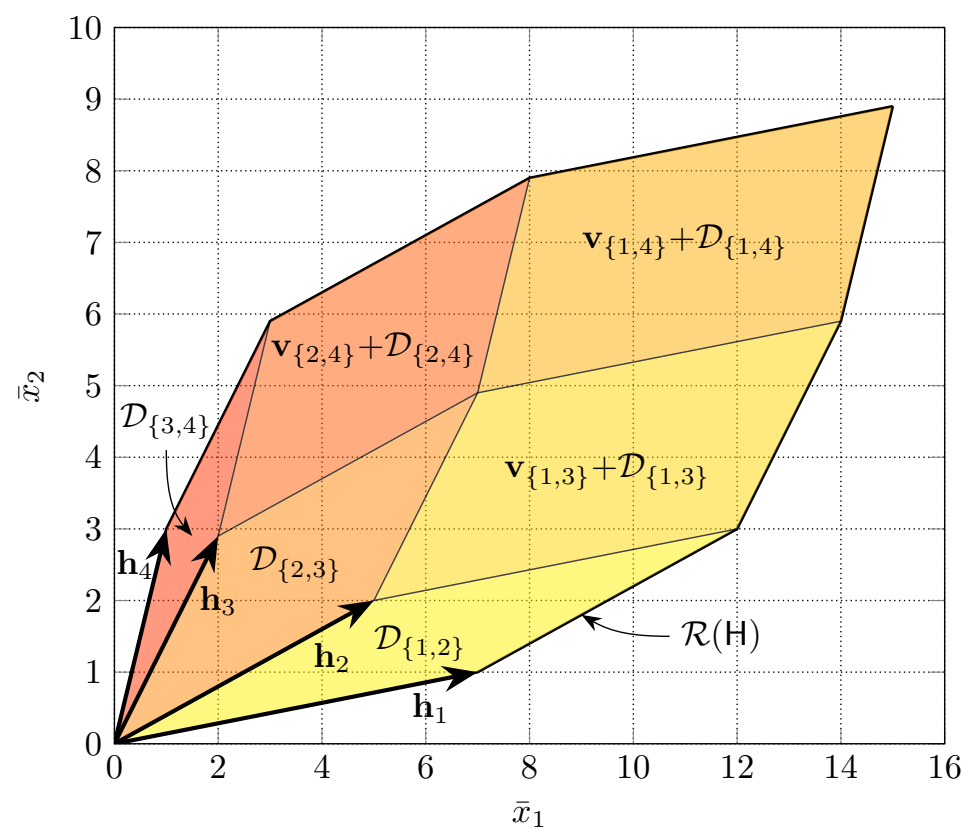

Figure 3: Partition of $\mathcal{R}(\mathrm{H})$ into the union (40) for the $2 \times 4$ MIMO channel matrix $\mathbf{H}=[7,5,2,1 ; 1,2,2.9,3]$. The peak power is $\mathrm{A}=1$.

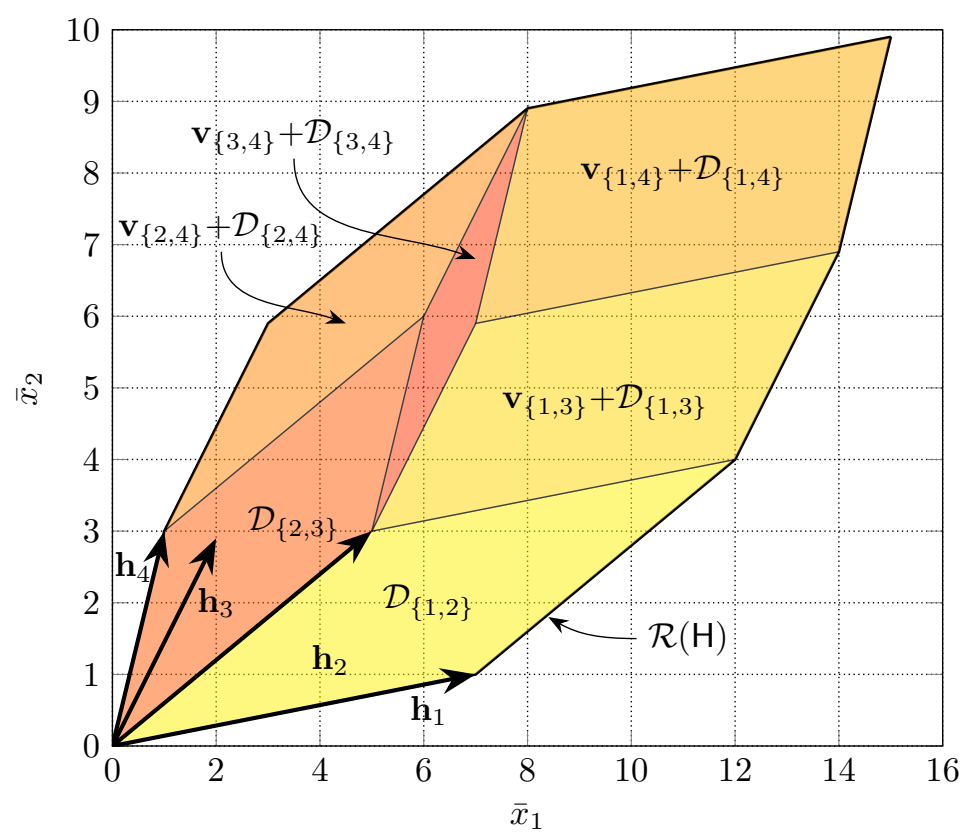

Figure 4: Partition of $\mathcal{R}(\mathrm{H})$ into the union (40) for the $2 \times 4$ MIMO channel matrix $\mathbf{H}=[7,5,2,1 ; 1,3,2.9,3]$. The peak power is $\mathrm{A}=1$. 


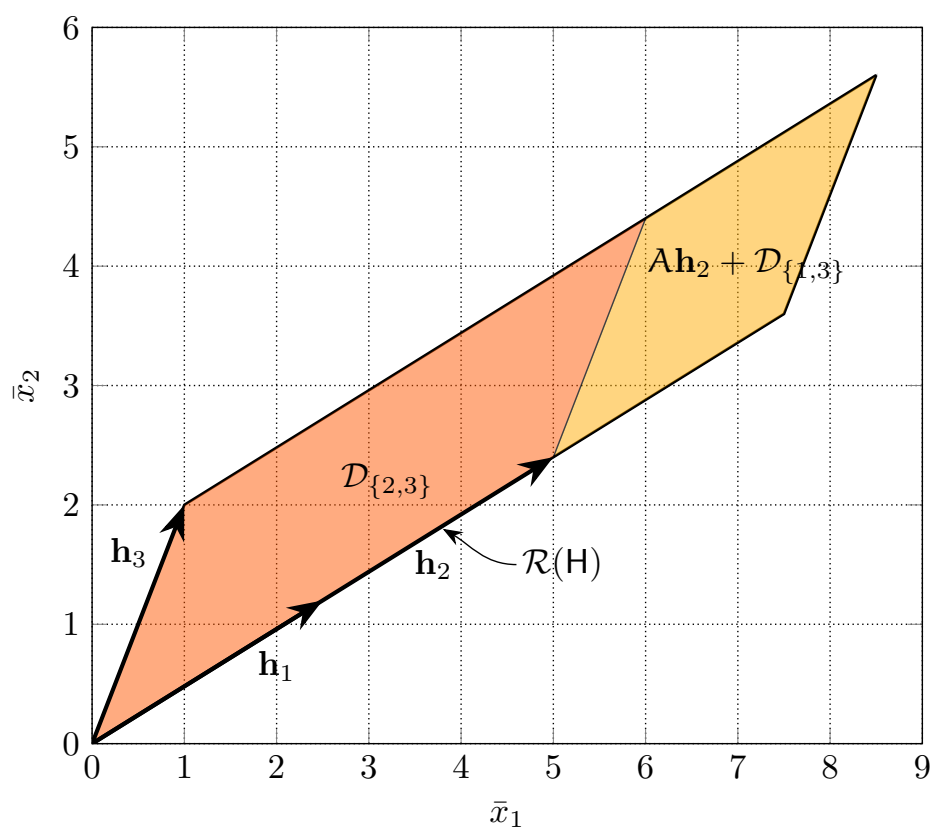

Figure 5: The zonotope $\mathcal{R}(\mathrm{H})$ for the $2 \times 3$ MIMO channel matrix $\mathrm{H}=[2.5,5,1 ; 1.2,2.4,2]$ and its minimum-energy decomposition into two parallelograms. The peak power is $A=1$.

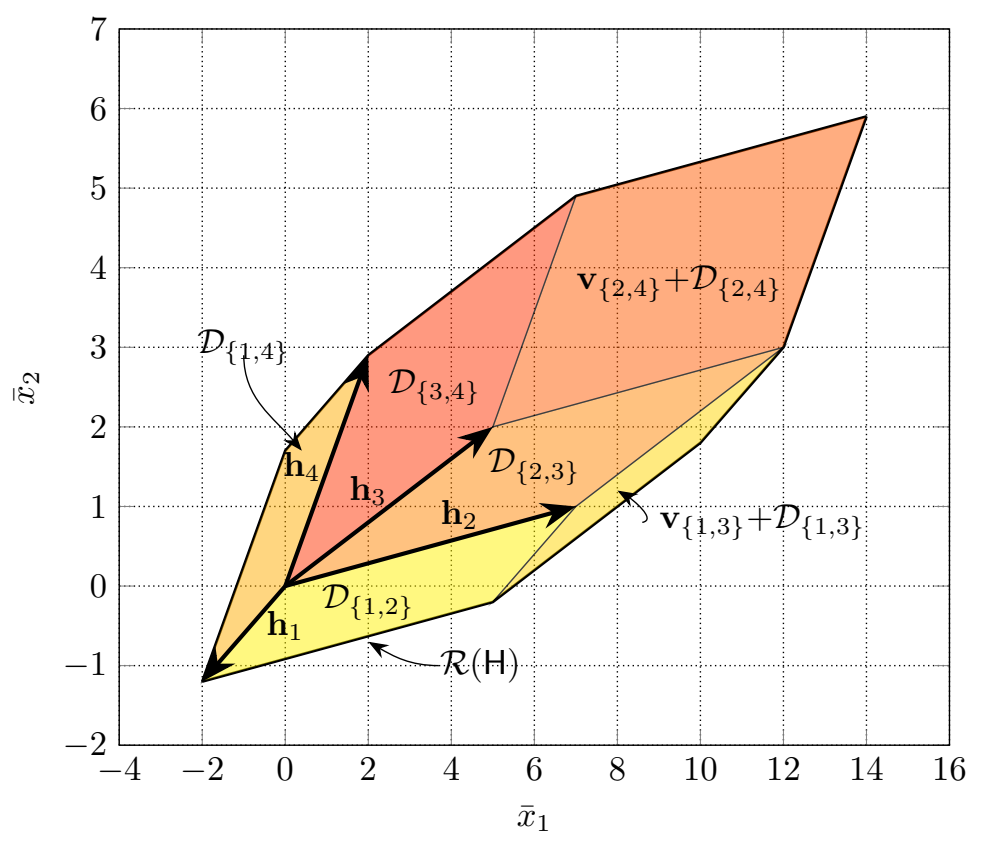

Figure 6: Partition of $\mathcal{R}(\mathrm{H})$ into the union (40) for the $2 \times 4$ MIMO channel matrix $\mathbf{H}=[-2,7,5,2 ;-1.2,1,2,2.9]$. The peak power is $\mathrm{A}=1$. 
Remark 6. If all $n_{\mathrm{R}}$ column vectors in $\mathrm{H}$ are linearly independent, then the minimumenergy signaling partitions the range of $\overline{\mathbf{x}}$ into $\left(\begin{array}{l}n_{\mathrm{T}} \\ n_{\mathrm{R}}\end{array}\right)$ parallelepipeds. If some column vectors in $\mathrm{H}$ are linearly dependent, the number of parallelepipeds in the minimum-energy signaling partitioning will be less than $\left(\begin{array}{l}n_{\mathrm{T}} \\ n_{\mathrm{R}}\end{array}\right)$. Indeed, the number of parallelepipeds is equal to the number of ways of choosing $n_{\mathrm{R}}$ linearly independent column vectors from $\mathrm{H}$.

Figure 5 shows an example of a $2 \times 3$ MIMO channel with $\mathbf{h}_{1}$ and $\mathbf{h}_{2}$ being linearly dependent $\left(\mathbf{h}_{2}=2 \mathbf{h}_{1}\right)$. Instead of the usual $\left(\begin{array}{l}3 \\ 2\end{array}\right)=3$ different parallelepipeds, in this example there are only two.

Note that Lemma 5 holds true irrespectively of the number of parallelepipeds.

\section{Equivalent Capacity Expression}

We are now going to state an alternative expression for the capacity $C_{H}(A, \alpha A)$ in terms of $\overline{\mathbf{X}}$ instead of $\mathbf{X}$. To that goal we define for each index set $\mathcal{U} \in \mathscr{U}$

$$
s_{\mathcal{U}} \triangleq \sum_{j \in \mathcal{U}^{\mathrm{c}}} g_{\mathcal{U}, j}, \quad \mathcal{U} \in \mathscr{U}
$$

which indicates the number of components of the input vector that are set to $A$ in order to induce $\mathbf{v}_{\mathcal{U}}$ in $(39)$.

Remark 7. It follows directly from Lemma 5 that, for every $\mathcal{U} \in \mathscr{U}$,

$$
0 \leq s_{\mathcal{U}} \leq n_{\mathrm{T}}-n_{\mathrm{R}}
$$

We define a random variable $\mathbb{U}$ over $\mathscr{U}$ to indicate which parallelepiped $\overline{\mathbf{X}}$ belongs to, i.e.,

$$
(\mathbb{U}=\mathcal{U}) \Longrightarrow\left(\overline{\mathbf{X}} \in\left(\mathbf{v}_{\mathcal{U}}+\mathcal{D}_{\mathcal{U}}\right)\right)
$$

The choice of $\mathbb{U}$ that satisfies (46) is not unique because of the ambiguity at the boundary points of the different parallelepipeds: when $\overline{\mathbf{x}}$ takes a value on the boundary between multiple parallelepipeds, $\mathbb{U}$ could be randomly chosen among these parallelepipeds or deterministically set to one of them. However, the difference between these choices has no influence on our results, since the set of all boundary points has zero $n_{\mathrm{R}}$-dimensional Lebesgue measure. For clarity, we shall restrict $\mathbb{U}$ to being a deterministic function of $\overline{\mathbf{X}}$.

Proposition 8. The capacity $\mathrm{C}_{\mathrm{H}}(\mathrm{A}, \alpha \mathrm{A})$ as in (11) can be written as

$$
\mathrm{C}_{\mathrm{H}}(\mathrm{A}, \alpha \mathrm{A})=\max _{P_{\overline{\mathbf{X}}}} \mathrm{I}(\overline{\mathbf{X}} ; \mathbf{Y})
$$

where the maximization is over all distributions $P_{\overline{\mathbf{X}}}$ over $\mathcal{R}(\mathrm{H})$ subject to the power constraint:

$$
\mathrm{E}_{\mathbb{U}}\left[\mathrm{A} s_{\mathbb{U}}+\left\|\mathrm{H}_{\mathbb{U}}^{-1}\left(\mathrm{E}[\overline{\mathbf{X}} \mid \mathbb{U}]-\mathbf{v}_{\mathbb{U}}\right)\right\|_{1}\right] \leq \alpha \mathrm{A}
$$

where the random variable $\mathbb{U}$ is a function of $\overline{\mathbf{X}}$ that satisfies (46).

Proof: Notice that $\overline{\mathbf{X}}$ is a function of $\mathbf{X}$ and that we have a Markov chain $\mathbf{X} \multimap-$ $\overline{\mathbf{X}} \multimap \mathbf{Y}$. Therefore, $\mathrm{I}(\overline{\mathbf{X}} ; \mathbf{Y})=\mathrm{I}(\mathbf{X} ; \mathbf{Y})$. Moreover, by Lemma 5 , the range of $\overline{\mathbf{X}}$ in $\mathcal{R}(\mathrm{H})$ can be decomposed into the shifted parallelepipeds $\left\{\mathbf{v}_{\mathcal{U}}+\mathcal{D}_{\mathcal{U}}\right\}_{\mathcal{U} \in \mathscr{U}}$. Again by Lemma 5, for any image point $\overline{\mathbf{x}}$ in $\mathbf{v}_{\mathcal{U}}+\mathcal{D}_{\mathcal{U}}$, the minimum energy required to induce $\overline{\mathbf{x}}$ is

$$
A s_{\mathcal{U}}+\left\|\mathrm{H}_{\mathcal{U}}^{-1}\left(\overline{\mathbf{x}}-\mathbf{v}_{\mathcal{U}}\right)\right\|_{1}
$$


Without loss in optimality, we restrict ourselves to input vectors $\mathbf{x}$ that achieve some $\overline{\mathbf{x}}$ with minimum energy. Then, using $p_{\mathcal{U}}$ to denote $\operatorname{Pr}[\mathbb{U}=\mathcal{U}]$ and by the law of total expectation, the average power can be rewritten as

$$
\begin{aligned}
\mathrm{E}\left[\|\mathbf{X}\|_{1}\right] & =\sum_{\mathcal{U} \in \mathscr{U}} p_{\mathcal{U}} \mathrm{E}\left[\|\mathbf{X}\|_{1} \mid \mathbb{U}=\mathcal{U}\right] \\
& =\sum_{\mathcal{U} \in \mathscr{U}} p_{\mathcal{U}} \mathrm{E}\left[\mathrm{A} s_{\mathcal{U}}+\left\|\mathrm{H}_{\mathcal{U}}^{-1}\left(\overline{\mathbf{X}}-\mathbf{v}_{\mathcal{U}}\right)\right\|_{1} \mid \mathbb{U}=\mathcal{U}\right] \\
& =\sum_{\mathcal{U} \in \mathscr{U}} p_{\mathcal{U}}\left(A s_{\mathcal{U}}+\mathrm{E}\left[\left\|\mathrm{H}_{\mathcal{U}}^{-1}\left(\overline{\mathbf{X}}-\mathbf{v}_{\mathcal{U}}\right)\right\|_{1} \mid \mathbb{U}=\mathcal{U}\right]\right) \\
& =\sum_{\mathcal{U} \in \mathscr{U}} p_{\mathcal{U}}\left(\mathrm{A} s_{\mathcal{U}}+\left\|\mathrm{H}_{\mathcal{U}}^{-1}\left(\mathrm{E}[\overline{\mathbf{X}} \mid \mathbb{U}=\mathcal{U}]-\mathbf{v}_{\mathcal{U}}\right)\right\|_{1}\right) \\
& =\mathrm{E}_{\mathbb{U}}\left[\mathrm{A}_{s_{\mathbb{U}}}+\left\|\mathrm{H}_{\mathbb{U}}^{-1}\left(\mathrm{E}[\overline{\mathbf{X}} \mid \mathbb{U}]-\mathbf{v}_{\mathbb{U}}\right)\right\|_{1}\right],
\end{aligned}
$$

where (53) follows from the fact that all components of $\mathrm{H}_{\mathcal{U}}^{-1}\left(\overline{\mathbf{X}}-\mathbf{v}_{\mathcal{U}}\right)$ are nonnegative.

Remark 9. The term inside the expectation on the left-hand side (LHS) of (48) can be seen as a cost function for $\overline{\mathbf{X}}$, where the cost is linear within each of the parallelepipeds $\left\{\mathcal{D}_{\mathcal{U}}+\mathbf{v}_{\mathcal{U}}\right\}_{\mathcal{U} \in \mathscr{U}}$ (but not linear on the entire $\mathcal{R}(\mathrm{H})$ ). At very high $\mathrm{SNR}$, the receiver can obtain an almost perfect guess of $\mathbb{U}$. As a result, our channel can be seen as a set of almost parallel channels in the sense of [27, Exercise 7.28]. Each one of the parallel channels is an amplitude-constrained $n_{\mathrm{R}} \times n_{\mathrm{R}}$ MIMO channel, with a linear power constraint. This observation will help us obtain upper and lower bounds on capacity that are tight in the high-SNR limit. Specifically, for an upper bound, we reveal $\mathbb{U}$ to the receiver and then apply previous results on full-rank $n_{\mathrm{R}} \times n_{\mathrm{R}}$ MIMO channels [16]. For a lower bound, we choose the inputs in such a way that, on each parallelepiped $\mathcal{D}_{\mathcal{U}}+\mathbf{v}_{\mathcal{U}}$, the vector $\overline{\mathbf{X}}$ has the high-SNR-optimal distribution for the corresponding $n_{\mathrm{R}} \times n_{\mathrm{R}}$ channel.

\section{Maximum-Variance Signaling}

The proofs to the lemmas in this section are given in Appendix D.

As we shall see (Theorem 22 ahead and [20], [28]), at low SNR the asymptotic capacity is characterized by the maximum trace of the covariance matrix of $\overline{\mathbf{X}}$, which we denote

$$
\mathrm{K}_{\overline{\mathbf{X}} \overline{\mathbf{X}}} \triangleq \mathrm{E}\left[(\overline{\mathbf{X}}-\mathrm{E}[\overline{\mathbf{X}}])(\overline{\mathbf{X}}-\mathrm{E}[\overline{\mathbf{X}}])^{\top}\right]
$$

In this section we discuss properties of an optimal input distribution for $\mathbf{X}$ that maximizes this trace. Thus, we are interested in the following maximization problem:

$$
\max _{P \mathbf{X} \text { satisfying (6) }} \operatorname{tr}\left(\mathrm{K}_{\overline{\mathbf{X}} \overline{\mathbf{X}})}\right.
$$

where the maximization is over all input distributions $P_{\mathbf{X}}$ satisfying the peak- and average-power constraints given in (6).

The following three lemmas show that the optimal input to the optimization problem in (56) has certain structures: Lemma 10 shows that it is discrete with all entries of mass points taking values in $\{0, A\}$; Lemma 11 shows that the possible values of the optimal $\mathbf{X}$ form a "path" in $[0, A]^{n_{\mathrm{T}}}$ starting from the origin; and Lemma 12 shows that, under mild assumptions, this optimal $\mathbf{X}$ takes at most $n_{\mathrm{R}}+2$ values.

Lemma 10. An optimal input to the maximization problem in (56) uses for each component of $\mathbf{X}$ only the values 0 and $A$ :

$$
\operatorname{Pr}\left[X_{i} \in\{0, \mathrm{~A}\}\right]=1, \quad i=1, \ldots, n_{\mathrm{T}} .
$$


Lemma 11. An optimal input to the optimization problem in (56) is a PMF $P_{\mathbf{X}}^{*}$ over a set $\left\{\mathbf{x}_{1}^{*}, \mathbf{x}_{2}^{*}, \ldots\right\}$ satisfying

$$
x_{k, \ell}^{*} \leq x_{k^{\prime}, \ell}^{*} \text { for all } k<k^{\prime}, \ell=1, \ldots, n_{\mathrm{T}} .
$$

Furthermore, the first point is $\mathbf{x}_{1}^{*}=\mathbf{0}$, and

$$
P_{\mathbf{X}}^{*}(\mathbf{0})>0 .
$$

Notice that Lemma 10 and the first part of Lemma 11 have already been proven in [20]. A proof is given in the appendix for completeness.

Define $\mathscr{T}$ to be the power set of $\left\{1, \ldots, n_{\mathrm{T}}\right\}$ without the empty set, and define for every $\mathcal{V} \in \mathscr{T}$ and every $i \in\left\{1, \ldots, n_{\mathrm{R}}\right\}$

$$
r_{\mathcal{V}, i} \triangleq \sum_{k=1}^{n_{\mathrm{T}}} h_{i, k} \mathbb{1}\{k \in \mathcal{V}\}, \quad \forall \mathcal{V} \in \mathscr{T}, \forall i \in\left\{1, \ldots, n_{\mathrm{R}}\right\},
$$

with $\mathbb{1}\{\cdot\}$ denoting the indicator function. (Here $\mathcal{V}$ describes a certain choice of input antennas that will be set to $A$, while the remaining antennas will be set to 0 .) Number all possible $\mathcal{V} \in \mathscr{T}$ from $\mathcal{V}_{1}$ to $\mathcal{V}_{\mathrm{T}}\left(\right.$ where $\left.\mathrm{T}=2^{n_{\mathrm{T}}}-1\right)$ and define the matrix

$$
\mathrm{R} \triangleq\left(\begin{array}{ccccc}
2 r_{\mathcal{V}_{1}, 1} & \cdots & 2 r_{\mathcal{V}_{1}, n_{\mathrm{R}}} & \left|\mathcal{V}_{1}\right| & \left\|\mathbf{r}_{\mathcal{V}_{1}}\right\|_{2}^{2} \\
2 r_{\mathcal{V}_{2}, 1} & \cdots & 2 r_{\mathcal{V}_{2}, n_{\mathrm{R}}} & \left|\mathcal{V}_{2}\right| & \left\|\mathbf{r}_{\mathcal{V}_{2}}\right\|_{2}^{2} \\
\vdots & \ddots & \vdots & \vdots & \vdots \\
2 r_{\mathcal{V}_{\mathrm{T}}, 1} & \cdots & 2 r_{\mathcal{V}_{\mathrm{T}}, n_{\mathrm{R}}} & \left|\mathcal{V}_{\mathrm{T}}\right| & \left\|\mathbf{r}_{\mathcal{V}_{\mathrm{T}}}\right\|_{2}^{2}
\end{array}\right)
$$

where

$$
\mathbf{r}_{\mathcal{V}} \triangleq\left(r_{\mathcal{V}, 1}, r_{\mathcal{V}, 2}, \ldots, r_{\mathcal{V}, n_{\mathrm{R}}}\right)^{\top}, \quad \forall \mathcal{V} \in \mathscr{T}
$$

Lemma 12. If every $\left(n_{\mathrm{R}}+2\right) \times\left(n_{\mathrm{R}}+2\right)$ submatrix $\mathrm{R}_{n_{\mathrm{R}}+2}$ of matrix $\mathrm{R}$ is of full rank

$$
\operatorname{rank}\left(\mathrm{R}_{n_{\mathrm{R}}+2}\right)=n_{\mathrm{R}}+2,
$$

then the optimal input to the optimization problem in (56) is a PMF $P_{\mathbf{X}}^{*}$ over a set $\left\{\mathbf{0}, \mathbf{x}_{1}^{*}, \ldots, \mathbf{x}_{n_{\mathrm{R}}+1}^{*}\right\}$ with $n_{\mathrm{R}}+2$ points.

Remark 13. Lemmas 5 and 10 together imply that the optimal $\overline{\mathbf{X}}$ in (56) takes value only in the set $\mathcal{F}_{\mathrm{CP}}$ of corner points of the parallelepipeds $\left\{\mathbf{v}_{\mathcal{U}}+\mathcal{D}_{\mathcal{U}}\right\}$ :

$$
\mathcal{F}_{\mathrm{CP}} \triangleq \bigcup_{\mathcal{U} \in \mathscr{U}}\left\{\mathbf{v}_{\mathcal{U}}+\sum_{i \in \mathcal{U}} \lambda_{i} \mathbf{h}_{i}: \lambda_{i} \in\{0, A\}, \forall i \in \mathcal{U}\right\} .
$$

Lemmas 11 and 12 further imply that the possible values of this optimal $\overline{\mathbf{X}}$ form a path in $\mathcal{F}_{\mathrm{CP}}$, starting from $\mathbf{0}$, and containing no more than $n_{\mathrm{R}}+2$ points.

Table 1 shows seven examples of distributions that maximize the trace of the covariance matrix, which fall into five different cases in terms of mass-point placement: there are only two mass points, one at $(0,0,0,0)$ and the other at $(A, A, A, A),(A, A, A, 0)$, or $(A, A, 0,0)$; or there are three mass points, the first at at $(0,0,0,0)$, the second at $(A, A, A, 0)$, and the third at either $(A, A, 0,0)$ or $(A, A, A, A)$. As expected, the distributions follow the properties claimed in Lemmas 10, 11, and 12. 
Table 1: Maximum variance distributions for different channel coefficients

\begin{tabular}{|c|c|c|c|c|c|c|c|}
\hline \multicolumn{5}{|c|}{ channel gains } & $\alpha$ & $\max _{P \mathbf{x}} \operatorname{tr}\left(\mathrm{K}_{\overline{\mathbf{X}} \overline{\mathbf{X}}}\right)$ & $P_{\mathbf{X}}: \max _{P_{\mathbf{X}}} \operatorname{tr}\left(\mathrm{K}_{\overline{\mathbf{X}} \overline{\mathbf{X}}}\right)$ \\
\hline $\mathrm{H}=$ & $\left(\begin{array}{l}1.3 \\
2.1\end{array}\right)$ & $\begin{array}{l}0.6 \\
4.5\end{array}$ & $\begin{array}{c}1 \\
0.7\end{array}$ & $\left.\begin{array}{l}0.1 \\
0.5\end{array}\right)$ & 1.5 & $16.3687 A^{2}$ & $\begin{array}{l}P_{\mathbf{X}}(0,0,0,0)=0.625 \\
P_{\mathbf{X}}(A, A, A, A)=0.375\end{array}$ \\
\hline $\mathrm{H}=$ & $\left(\begin{array}{l}1.3 \\
2.1\end{array}\right)$ & $\begin{array}{l}0.6 \\
4.5\end{array}$ & $\begin{array}{c}1 \\
0.7\end{array}$ & $\left.\begin{array}{l}0.1 \\
0.5\end{array}\right)$ & 0.9 & $12.957 A^{2}$ & $\begin{array}{l}P_{\mathbf{X}}(0,0,0,0)=0.7 \\
P_{\mathbf{X}}(\mathrm{A}, \mathrm{A}, \mathrm{A}, 0)=0.3\end{array}$ \\
\hline $\mathrm{H}=$ & $\left(\begin{array}{l}1.3 \\
2.1\end{array}\right)$ & $\begin{array}{l}0.6 \\
4.5\end{array}$ & $\begin{array}{c}1 \\
0.7\end{array}$ & $\left.\begin{array}{l}0.1 \\
0.5\end{array}\right)$ & 0.6 & $9.9575 A^{2}$ & $\begin{array}{l}P_{\mathbf{X}}(0,0,0,0)=0.7438 \\
P_{\mathbf{X}}(A, A, 0,0)=0.1687 \\
P_{\mathbf{X}}(A, A, A, 0)=0.0875\end{array}$ \\
\hline $\mathrm{H}=$ & $\left(\begin{array}{l}1.3 \\
2.1\end{array}\right)$ & $\begin{array}{l}0.6 \\
4.5\end{array}$ & $\begin{array}{c}1 \\
0.7\end{array}$ & $\left.\begin{array}{l}0.1 \\
0.5\end{array}\right)$ & 0.3 & $6.0142 A^{2}$ & $\begin{array}{l}P_{\mathbf{X}}(0,0,0,0)=0.85 \\
P_{\mathbf{X}}(A, A, 0,0)=0.15\end{array}$ \\
\hline $\mathrm{H}=$ & $\left(\begin{array}{l}0.9 \\
0.5 \\
0.7\end{array}\right.$ & $\begin{array}{l}3.2 \\
3.5 \\
1.1\end{array}$ & $\begin{array}{c}1 \\
1.7 \\
1.1\end{array}$ & $\left.\begin{array}{l}2.1 \\
2.5 \\
1.3\end{array}\right)$ & 0.9 & $23.8405 A^{2}$ & $\begin{array}{l}P_{\mathbf{X}}(0,0,0,0)=0.7755 \\
P_{\mathbf{X}}(\text { A }, A, A, A)=0.2245\end{array}$ \\
\hline $\mathrm{H}=$ & $\left(\begin{array}{l}0.9 \\
0.5 \\
0.7\end{array}\right.$ & $\begin{array}{l}3.2 \\
3.5 \\
1.1\end{array}$ & $\begin{array}{c}1 \\
1.7 \\
1.1\end{array}$ & $\left.\begin{array}{l}2.1 \\
2.5 \\
1.3\end{array}\right)$ & 0.75 & $20.8950 A^{2}$ & $\begin{array}{l}P_{\mathbf{X}}(0,0,0,0)=0.7772 \\
P_{\mathbf{X}}(A, A, A, 0)=0.1413 \\
P_{\mathbf{X}}(A, A, A, A)=0.0815\end{array}$ \\
\hline $\mathrm{H}=$ & $\left(\begin{array}{l}0.9 \\
0.5 \\
0.7\end{array}\right.$ & $\begin{array}{l}3.2 \\
3.5 \\
1.1\end{array}$ & $\begin{array}{c}1 \\
1.7 \\
1.1\end{array}$ & $\left.\begin{array}{l}2.1 \\
2.5 \\
1.3\end{array}\right)$ & 0.6 & $17.7968 A^{2}$ & $\begin{array}{l}P_{\mathbf{X}}(0,0,0,0)=0.8 \\
P_{\mathbf{X}}(\mathrm{A}, \mathrm{A}, \mathrm{A}, 0)=0.2\end{array}$ \\
\hline
\end{tabular}




\section{Capacity Results}

Define

$$
\mathrm{V}_{\mathrm{H}} \triangleq \sum_{\mathcal{U} \in \mathscr{U}}\left|\operatorname{det} \mathrm{H}_{\mathcal{U}}\right| .
$$

Let $\mathbf{q}$ be a probability vector on $\mathscr{U}$ with entries

$$
q_{\mathcal{U}} \triangleq \frac{\left|\operatorname{det} \mathrm{H}_{\mathcal{U}}\right|}{\mathrm{V}_{\mathrm{H}}}, \quad \mathcal{U} \in \mathscr{U} .
$$

Further, define

$$
\alpha_{\mathrm{th}} \triangleq \frac{n_{\mathrm{R}}}{2}+\sum_{\mathcal{U} \in \mathscr{U}} s_{\mathcal{U}} q_{\mathcal{U}},
$$

where $\left\{s_{\mathcal{U}}\right\}$ are defined in (44). Notice that $\alpha_{\text {th }}$ determines the threshold value for $\alpha$ above which $\overline{\mathbf{X}}$ can be made uniform over $\mathcal{R}(\mathrm{H})$. In fact, combining the minimum-energy signaling in (42) with a uniform distribution for $\overline{\mathbf{X}}$ over $\mathcal{R}(\mathrm{H})$, the expected input power is

$$
\begin{aligned}
\mathrm{E}\left[\|\mathbf{X}\|_{1}\right] & =\sum_{\mathcal{U} \in \mathscr{U}} \operatorname{Pr}[\mathbb{U}=\mathcal{U}] \cdot \mathrm{E}\left[\|\mathbf{X}\|_{1} \mid \mathbb{U}=\mathcal{U}\right] \\
& =\sum_{\mathcal{U} \in \mathscr{U}} q_{\mathcal{U}}\left(\mathrm{A} s_{\mathcal{U}}+\frac{n_{\mathrm{R}} A}{2}\right) \\
& =\alpha_{\mathrm{th}} \mathcal{A}
\end{aligned}
$$

where the random variable $\mathbb{U}$ indicates the parallelepiped containing $\overline{\mathbf{X}}$; see (46). Equality (69) holds because, when $\overline{\mathbf{X}}$ is uniform over $\mathcal{R}(\mathbf{H}), \operatorname{Pr}[\mathbb{U}=\mathcal{U}]=q_{\mathcal{U}}$, and because, conditional on $\mathbb{U}=\mathcal{U}$, using the minimum-energy signaling scheme, the input vector $\mathbf{X}$ is uniform over $\mathbf{v}_{\mathcal{U}}+\mathcal{D}_{\mathcal{U}}$.

Remark 14. Note that

$$
\alpha_{\mathrm{th}} \leq \frac{n_{\mathrm{T}}}{2}
$$

as can be argued as follows. Let $\mathbf{X}$ be an input that achieves a uniform $\overline{\mathbf{X}}$ with minimum energy. According to (70) it consumes an input power $\alpha_{\mathrm{th}} \mathrm{A}$. Define $\mathbf{X}^{\prime}$ as

$$
X_{i}^{\prime} \triangleq \mathrm{A}-X_{i}, \quad i=1, \ldots, n_{\mathrm{T}} .
$$

It must consume input power $\left(n_{\mathrm{T}}-\alpha_{\mathrm{th}}\right)$ A. Note that $\mathbf{X}^{\prime}$ also induces a uniform $\overline{\mathbf{X}}$ because the zonotope $\mathcal{R}(\mathbf{H})$ is point-symmetric. Since $\mathbf{X}$ consumes minimum energy, we know

$$
\mathrm{E}\left[\|\mathbf{X}\|_{1}\right] \leq \mathrm{E}\left[\left\|\mathbf{X}^{\prime}\right\|_{1}\right]
$$

i.e.,

$$
\alpha_{\mathrm{th}} \mathrm{A} \leq\left(n_{\mathrm{T}}-\alpha_{\mathrm{th}}\right) \mathrm{A},
$$

which implies (71).

\subsection{Lower Bounds}

The proofs to the theorems in this section can be found in Appendix E. 
Theorem 15. If $\alpha \geq \alpha_{\mathrm{th}}$, then

$$
\mathrm{C}_{\mathrm{H}}(\mathrm{A}, \alpha \mathrm{A}) \geq \frac{1}{2} \log \left(1+\frac{A^{2 n_{\mathrm{R}}} V_{\mathrm{H}}^{2}}{(2 \pi e)^{n_{\mathrm{R}}}}\right) .
$$

Theorem 16. If $\alpha<\alpha_{\mathrm{th}}$, then

$$
\mathrm{C}_{\mathrm{H}}(\mathrm{A}, \alpha \mathrm{A}) \geq \frac{1}{2} \log \left(1+\frac{\mathrm{A}^{2 n_{\mathrm{R}}} \mathrm{V}_{\mathrm{H}}^{2}}{(2 \pi e)^{n_{\mathrm{R}}}} e^{2 \nu}\right)
$$

with

$$
\nu \triangleq \sup _{\lambda \in\left(\max \left\{0, \frac{n_{\mathrm{R}}}{2}+\alpha-\alpha_{\mathrm{th}}\right\}, \min \left\{\frac{n_{\mathrm{R}}}{2}, \alpha\right\}\right)}\left\{n_{\mathrm{R}}\left(1-\log \frac{\mu}{1-e^{-\mu}}-\frac{\mu e^{-\mu}}{1-e^{-\mu}}\right)-\inf _{\mathbf{p}} \mathrm{D}(\mathbf{p} \| \mathbf{q})\right\}
$$

where $\mu$ is the unique solution to the following equation:

$$
\frac{1}{\mu}-\frac{e^{-\mu}}{1-e^{-\mu}}=\frac{\lambda}{n_{\mathrm{R}}}
$$

and where the infimum is over all probability vectors $\mathbf{p}$ on $\mathscr{U}$ such that

$$
\sum_{\mathcal{U} \in \mathscr{U}} p_{\mathcal{U}} s_{\mathcal{U}}=\alpha-\lambda
$$

with $\left\{s_{\mathcal{U}}\right\}$ defined in (44).

The two lower bounds in Theorems 15 and 16 are derived by applying the EPI, and by maximizing the differential entropy $h(\overline{\mathbf{X}})$ under the constraint (48). When $\alpha \geq \alpha_{\text {th }}$, choosing $\overline{\mathbf{X}}$ to be uniformly distributed on $\mathcal{R}(\mathrm{H})$ satisfies (48), hence we can achieve $\mathrm{h}(\overline{\mathbf{X}})=\log \boldsymbol{V}_{\mathrm{H}}$. When $\alpha<\alpha_{\mathrm{th}}$, the uniform distribution is no longer an admissible distribution for $\overline{\mathbf{X}}$. In this case, we first select a PMF over the events $\{\overline{\mathbf{X}} \in$ $\left.\left(\mathbf{v}_{\mathcal{U}}+\mathcal{D}_{\mathcal{U}}\right)\right\}_{\mathcal{U} \in \mathscr{U}}$, and, given $\overline{\mathbf{X}} \in \mathbf{v}_{\mathcal{U}}+\mathcal{D}_{\mathcal{U}}$, we choose the inputs $\left\{X_{i}: i \in \mathcal{U}\right\}$ according to a truncated exponential distribution rotated by the matrix $\mathrm{H}_{\mathcal{U}}$. Interestingly, it is optimal to choose the truncated exponential distributions for all sets $\mathcal{U} \in \mathscr{U}$ to have the same parameter $\mu$. This parameter is determined by the power $\frac{\lambda}{n_{\mathrm{R}}} \mathrm{A}$ allocated to the $n_{\mathrm{R}}$ signaling inputs $\left\{X_{i}: i \in \mathcal{U}\right\}$.

\subsection{Upper Bounds}

The proofs to the theorems in this section can be found in Appendix F.

The first upper bound is based on an analysis of the channel with peak-power constraint only, i.e., the average-power constraint (6b) is ignored.

Theorem 17. For an arbitrary $\alpha$,

$$
\mathrm{C}_{\mathrm{H}}(\mathrm{A}, \alpha \mathrm{A}) \leq \sup _{\mathbf{p}}\left\{\log \mathrm{V}_{\mathrm{H}}-\mathrm{D}(\mathbf{p} \| \mathbf{q})+\sum_{\mathcal{U} \in \mathscr{U}} p_{\mathcal{U}} \sum_{\ell=1}^{n_{\mathrm{R}}} \log \left(\sigma_{\mathcal{U}, \ell}+\frac{\mathrm{A}}{\sqrt{2 \pi e}}\right)\right\},
$$

where $\sigma_{\mathcal{U}, \ell}$ denotes the square root of the $\ell$ th diagonal entry of the matrix $\mathrm{H}_{\mathcal{U}}^{-1} \mathrm{H}_{\mathcal{U}}^{-\top}$, and where the supremum is over all probability vectors $\mathbf{p}$ on $\mathscr{U}$.

The following two upper bounds in Theorems 18 and 19 hold only when $\alpha<\alpha_{\text {th }}$.

Theorem 18. If $\alpha<\alpha_{\mathrm{th}}$, then

$$
\mathrm{C}_{\mathrm{H}}(\mathrm{A}, \alpha \mathrm{A}) \leq \sup _{\mathbf{p}} \inf _{\mu>0}\left\{\log \mathrm{V}_{\mathrm{H}}-\mathrm{D}(\mathbf{p} \| \mathbf{q})+\sum_{\mathcal{U} \in \mathscr{U}} p_{\mathcal{U}} \sum_{\ell=1}^{n_{\mathrm{R}}} \log \left(\sigma_{\mathcal{U}, \ell}+\frac{\mathrm{A}}{\sqrt{2 \pi e}} \frac{1-e^{-\mu}}{\mu}\right)\right.
$$




$$
\left.+\frac{\mu}{A \sqrt{2 \pi}} \sum_{\mathcal{U} \in \mathscr{U}} p_{\mathcal{U}} \sum_{\ell=1}^{n_{\mathrm{R}}} \sigma_{\mathcal{U}, \ell}\left(1-e^{-\frac{\AA^{2}}{2 \sigma_{\mathcal{U}, \ell}^{2}}}\right)+\mu\left(\alpha-\sum_{\mathcal{U} \in \mathscr{U}} p_{\mathcal{U}} s_{\mathcal{U}}\right)\right\}
$$

where the supremum is over all probability vectors $\mathbf{p}$ on $\mathscr{U}$ such that

$$
\sum_{\mathcal{U} \in \mathscr{U}} p_{\mathcal{U}} s_{\mathcal{U}} \leq \alpha
$$

Theorem 19. If $\alpha<\alpha_{\mathrm{th}}$, then

$$
\begin{aligned}
& \mathrm{C}_{\mathrm{H}}(\mathrm{A}, \alpha \mathrm{A}) \\
& \leq \sup _{\mathbf{p}} \inf _{\delta, \mu>0}\left\{\log \mathbf{V}_{\mathbf{H}}-\mathrm{D}(\mathbf{p} \| \mathbf{q})+\sum_{\mathcal{U} \in \mathscr{U}} p_{\mathcal{U}} \sum_{\ell=1}^{n_{\mathrm{R}}} \log \left(A \cdot \frac{e^{\frac{\mu \delta}{A}}-e^{-\mu\left(1+\frac{\delta}{A}\right)}}{\sqrt{2 \pi e} \mu\left(1-2 \mathcal{Q}\left(\frac{\delta}{\sigma_{\mathcal{U}, \ell}}\right)\right)}\right)\right. \\
& +\sum_{\mathcal{U} \in \mathscr{U}} p_{\mathcal{U}} \sum_{\ell=1}^{n_{\mathrm{R}}} \mathcal{Q}\left(\frac{\delta}{\sigma_{\mathcal{U}, \ell}}\right)+\sum_{\mathcal{U} \in \mathscr{U}} p_{\mathcal{U}} \sum_{\ell=1}^{n_{\mathrm{R}}} \frac{\delta}{\sqrt{2 \pi} \sigma_{\mathcal{U}, \ell}} e^{-\frac{\delta^{2}}{2 \sigma_{\mathcal{U}, \ell}^{2}}} \\
& \left.+\frac{\mu}{A \sqrt{2 \pi}} \sum_{\mathcal{U} \in \mathscr{U}} p_{\mathcal{U}} \sum_{\ell=1}^{n_{\mathrm{R}}} \sigma_{\mathcal{U}, \ell}\left(e^{-\frac{\delta^{2}}{2 \sigma_{\mathcal{U}, \ell}^{2}}}-e^{-\frac{(\mathrm{A}+\delta)^{2}}{2 \sigma_{\mathcal{U}, \ell}^{2}}}\right)+\mu\left(\alpha-\sum_{\mathcal{U} \in \mathscr{U}} p_{\mathcal{U}} s_{\mathcal{U}}\right)\right\},
\end{aligned}
$$

where $\mathcal{Q}(\cdot)$ denotes the $\mathcal{Q}$-function associated with the standard normal distribution, and the supremum is over all probability vectors $\mathbf{p}$ on $\mathscr{U}$ satisfying (82).

The three upper bounds in Theorems 17, 18 and 19 are derived using the fact that capacity cannot be larger than over a channel where the receiver observes both $\mathbf{Y}$ and $\mathbb{U}$. The mutual information corresponding to this channel $\mathrm{I}(\overline{\mathbf{X}} ; \mathbf{Y}, \mathbb{U})$ decomposes as $\mathrm{H}(\mathbb{U})+\mathrm{I}(\overline{\mathbf{X}} ; \mathbf{Y} \mid \mathbb{U})$, where the term $\mathrm{H}(\mathbb{U})$ indicates the rate that can be achieved by coding over the choice of the parallelepiped to which $\overline{\mathbf{X}}$ belongs, and $\mathrm{I}(\overline{\mathbf{X}} ; \mathbf{Y} \mid \mathbb{U})$ indicates the average rate that can be achieved by coding over a single parallelepiped. By the results in Lemma 5, we can treat the channel matrix as an invertible matrix when knowing $\mathbb{U}$, which greatly simplifies the bounding on $\mathrm{I}(\overline{\mathbf{X}} ; \mathbf{Y} \mid \mathbb{U})$. The upper bounds are then obtained by optimizing over the probabilities assigned to the different parallelepipeds. As we will see later, the upper bounds are asymptotically tight at high SNR. The reason is that the additional term $\mathrm{I}(\overline{\mathbf{X}} ; \mathbf{Y}, \mathbb{U})-\mathrm{I}(\overline{\mathbf{X}} ; \mathbf{Y})=\mathrm{I}(\overline{\mathbf{X}} ; \mathbb{U} \mid \mathbf{Y})$ vanishes as the SNR grows large. To derive the asymptotic high-SNR capacity, we also use previous results in [16], which derived the high-SNR capacity of this channel when the channel matrix is invertible.

Our next upper bound in Theorem 20 is determined by the maximum trace of the covariance matrix of $\overline{\mathbf{X}}$ under constraints (6).

Theorem 20. For an arbitrary $\alpha$,

$$
\mathrm{C}_{\mathrm{H}}(\mathrm{A}, \alpha \mathrm{A}) \leq \frac{n_{\mathrm{R}}}{2} \log \left(1+\frac{1}{n_{\mathrm{R}}} \max _{P_{\mathbf{x}}} \operatorname{tr}\left(\mathrm{K}_{\overline{\mathbf{x}} \overline{\mathbf{x}}}\right)\right)
$$

where the maximization is over all input distributions $P_{\mathbf{X}}$ satisfying the power constraints (6).

Note that Section 5 provides results that considerably simplify the maximization in (84). In particular, there exists a maximizing $P_{\mathbf{X}}$ that is a PMF over $\mathbf{0}$ and at most $n_{\mathrm{R}}+1$ other points on $\mathcal{F}_{\mathrm{CP}}$, where $\mathcal{F}_{\mathrm{CP}}$ is defined in (64).

\subsection{Asymptotic Capacity Expressions}

The proofs to the theorems in this section can be found in Appendix G. 
Theorem 21 (High-SNR Asymptotics). If $\alpha \geq \alpha_{\mathrm{th}}$, then

$$
\lim _{A \rightarrow \infty}\left\{C_{H}(A, \alpha A)-n_{R} \log A\right\}=\frac{1}{2} \log \left(\frac{V_{H}^{2}}{(2 \pi e)^{n_{R}}}\right) \text {. }
$$

If $\alpha<\alpha_{\mathrm{th}}$, then

$$
\lim _{A \rightarrow \infty}\left\{C_{H}(A, \alpha A)-n_{R} \log A\right\}=\frac{1}{2} \log \left(\frac{V_{H}^{2}}{(2 \pi e)^{n_{R}}}\right)+\nu,
$$

where $\nu<0$ is defined in $(77)-(79)$.

Recall that $\alpha_{\text {th }}$ is a threshold that determines whether $\overline{\mathbf{X}}$ can be uniformly distributed over $\mathcal{R}(\mathrm{H})$ or not. When $\alpha<\alpha_{\text {th }}$, compared with the asymptotic capacity without active average-power constraint, the average-power constraint imposes a penalty on the channel capacity. This penalty is characterized by $\nu$ in (86). As shown in Figure $7, \nu$ is a increasing function of $\alpha$. When $\alpha<\alpha_{\mathrm{th}}, \nu$ is always negative, and increases to 0 when $\alpha \geq \alpha_{\text {th }}$.

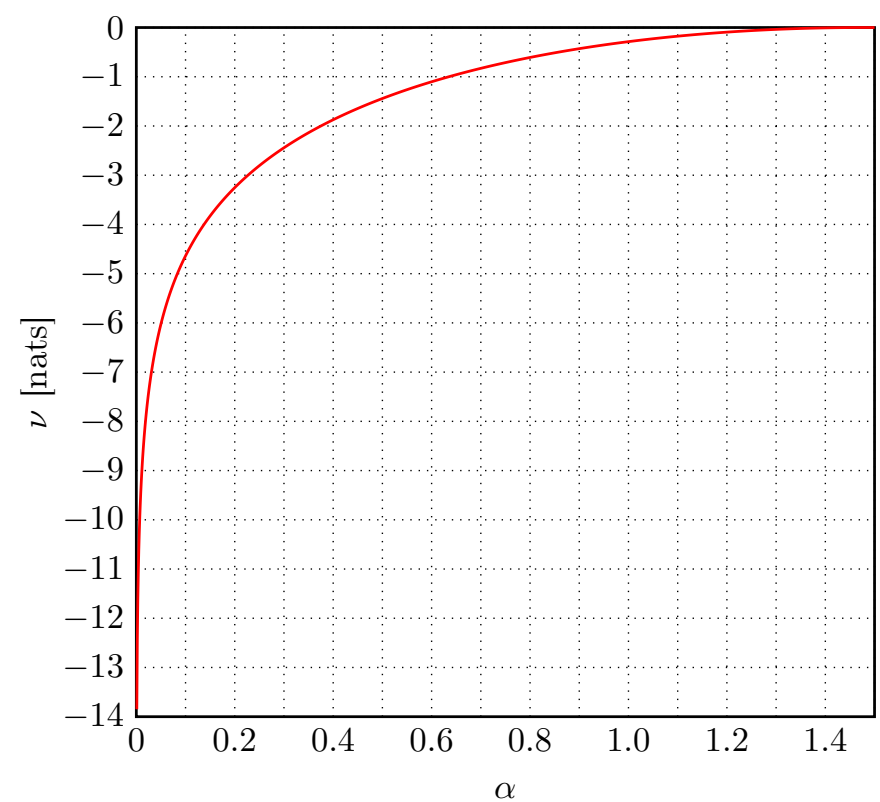

Figure 7: The parameter $\nu$ in (77) as a function of $\alpha$, for a $2 \times 3$ MIMO channel with channel matrix $\mathrm{H}=[1,1.5,3 ; 2,2,1]$ with corresponding $\alpha_{\mathrm{th}}=1.4762$. Recall that $\nu$ is the asymptotic capacity gap to the case with no active average-power constraint.

Theorem 22 (Low-SNR Asymptotics). For an arbitrary $\alpha$,

$$
\lim _{A \downarrow 0} \frac{C_{\mathrm{H}}(A, \alpha A)}{A^{2}}=\frac{1}{2} \max _{P_{\mathbf{X}}} \operatorname{tr}\left(\mathrm{K}_{\overline{\mathbf{X}} \overline{\mathbf{x}}}\right),
$$

where the maximization is over all input distributions $P_{\mathbf{X}}$ satisfying the constraints

$$
\begin{aligned}
\operatorname{Pr}\left[X_{k} \in[0,1]\right] & =1, \quad \forall k \in\left\{1, \ldots, n_{\mathrm{T}}\right\}, \\
\mathrm{E}\left[\|\mathbf{X}\|_{1}\right] & \leq \alpha .
\end{aligned}
$$

Again, see the results in Section 5 about maximizing the trace of the covariance matrix $\mathrm{K}_{\overline{\mathbf{X}} \overline{\mathbf{x}}}$.

Example 23. Figure 8 plots the asymptotic slope, i.e., the right-hand side (RHS) of (87), as a function of $\alpha$ for a $2 \times 3$ MIMO channel. As we can see, the asymptotic slope is strictly increasing for all values of $\alpha<\frac{n_{\mathrm{T}}}{2}$. 


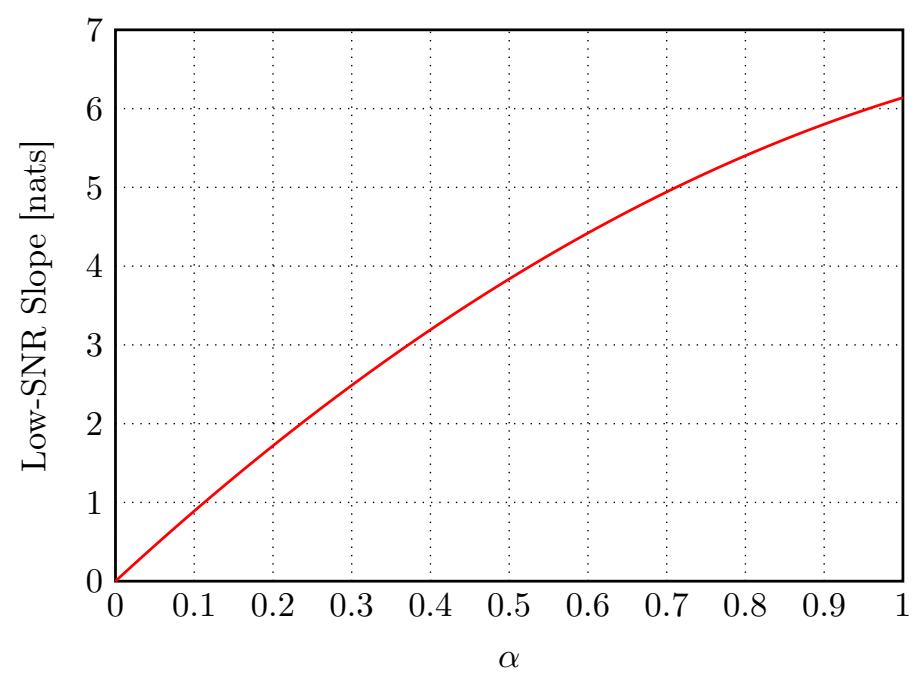

Figure 8: Low-SNR slope as a function of $\alpha$, for a $2 \times 3$ MIMO channel with channel matrix $\mathrm{H}=[1,1.5,3 ; 2,2,1]$.

\subsection{Numerical Results}

In the following we present some numerical examples of our lower and upper bounds.

Example 24. Figures 9 and 10 depict the derived lower and upper bounds for a $2 \times 3$ MIMO channel (same channel as in Example 23) for $\alpha=0.9$ and $\alpha=0.3$ (both values are less than $\left.\alpha_{\mathrm{th}}=1.4762\right)$, respectively. Both upper bounds (81) and (83) match with lower bound (76) asymptotically as A tends to infinity. Moreover, upper bound (80) gives a good approximation on capacity when the average-power constraint is weak (i.e., when $\alpha$ is close to $\alpha_{\mathrm{th}}$ ). Indeed, (80) is asymptotically tight at high SNR when $\alpha \geq \alpha_{\mathrm{th}}$. We also plot three numerical lower bounds obtained by optimizing $\mathrm{I}(\overline{\mathbf{X}} ; \mathbf{Y})$ over all feasible choices of $\overline{\mathbf{X}}$ that have positive probability on two, three, or four distinct mass points. (One of the mass points is always at $\mathbf{0}$.) In the low-SNR regime, upper bound (84) matches well with the two-point numerical lower bound. Actually (84) shares the same slope with capacity when the SNR tends to zero, which can be seen by comparing (84) with Theorem 22.

Example 25. Figures 11 and 12 show similar trends in a $2 \times 4$ MIMO channel. Note that although in the $2 \times 3$ channel of Figures 9 and 10 the upper bound (81) is always tighter than (83), this does not hold in general, as can be seen in Figure 12.

\section{Concluding Remarks}

In this paper, we first express capacity as a maximization problem over distributions for the vector $\overline{\mathbf{X}}=\mathbf{H X}$. The main challenge there is to transform the total average-power constraint on $\mathbf{X}$ to a constraint on $\overline{\mathbf{X}}$, as the mapping from $\mathbf{x}$ to $\overline{\mathbf{x}}$ is many-to-one. This problem is solved by identifying, for each $\overline{\mathbf{x}}$, the input vector $\mathbf{x}_{\min }$ that induces this $\overline{\mathbf{x}}$ with minimum energy. Specifically, we show that the set $\mathcal{R}(\mathrm{H})$ of all possible $\overline{\mathbf{x}}$ can be decomposed into a number of parallelepipeds such that, for all $\overline{\mathbf{x}}$ within one parallelepiped, the minimum-energy input vectors $\mathbf{x}_{\min }$ have a similar form.

At high SNR, the above minimum-energy signaling result allows the transmitter to decompose the channel into several "almost parallel" channels, each of which being an $n_{\mathrm{R}} \times n_{\mathrm{R}}$ MIMO channel in itself. This is because, at high SNR, the output $\mathbf{Y}$ allows the receiver to obtain a good estimate of which of the parallelepipeds $\overline{\mathbf{X}}$ lies in. We 


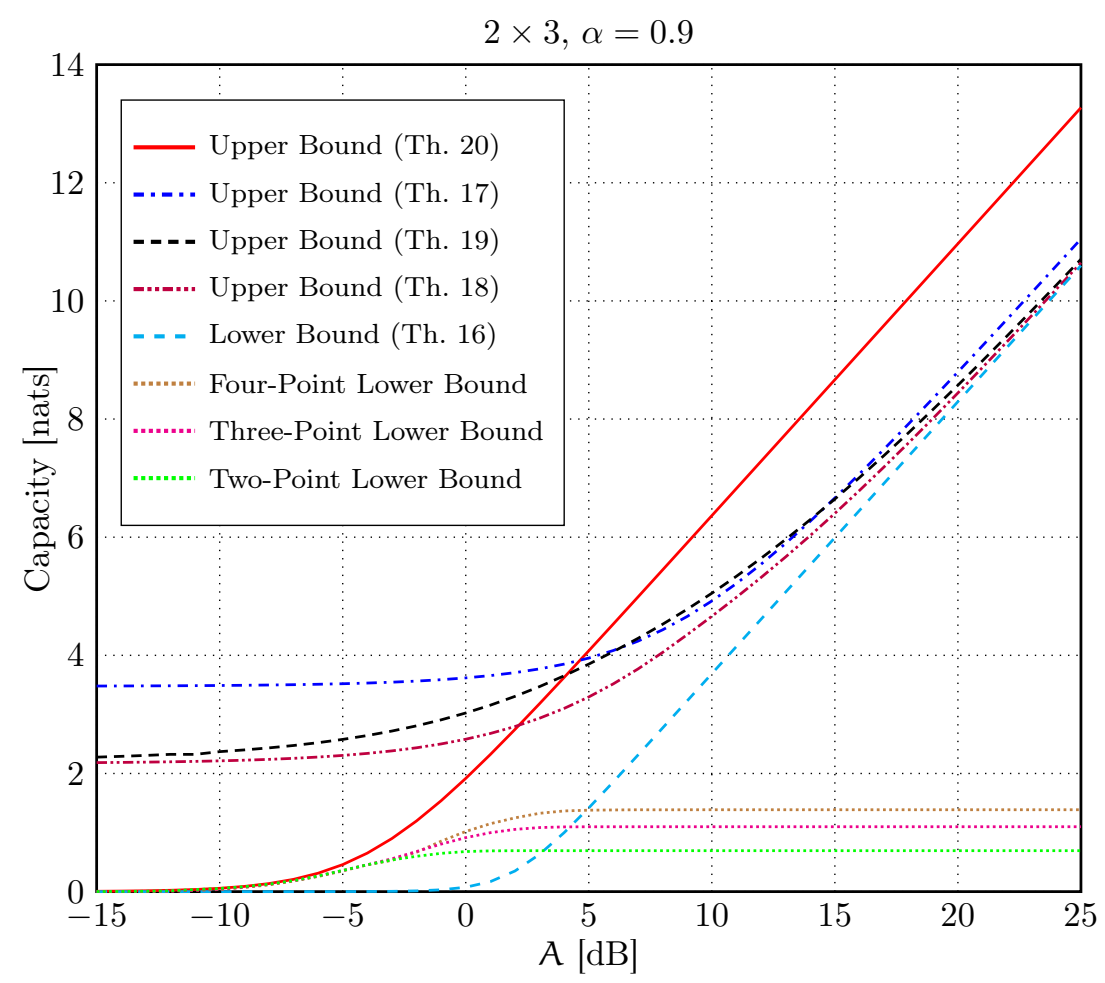

Figure 9: Bounds on capacity of $2 \times 3$ MIMO channel with channel matrix $\mathrm{H}=$ $[1,1.5,3 ; 2,2,1]$, and average-to-peak power ratio $\alpha=0.9$. Note that the threshold of the channel is $\alpha_{\mathrm{th}}=1.4762$.

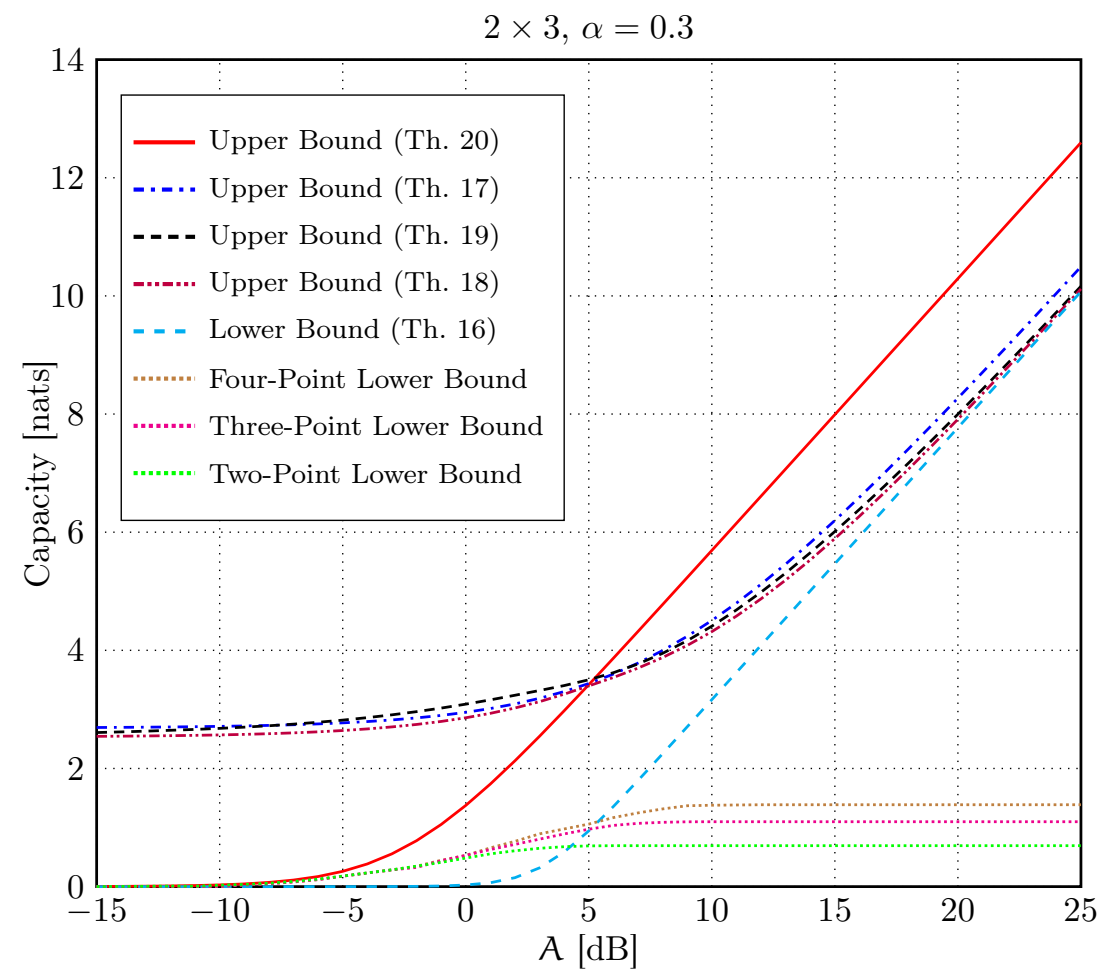

Figure 10: Bounds on capacity of the same $2 \times 3$ MIMO channel as discussed in Figure 9, and average-to-peak power ratio $\alpha=0.3$. 


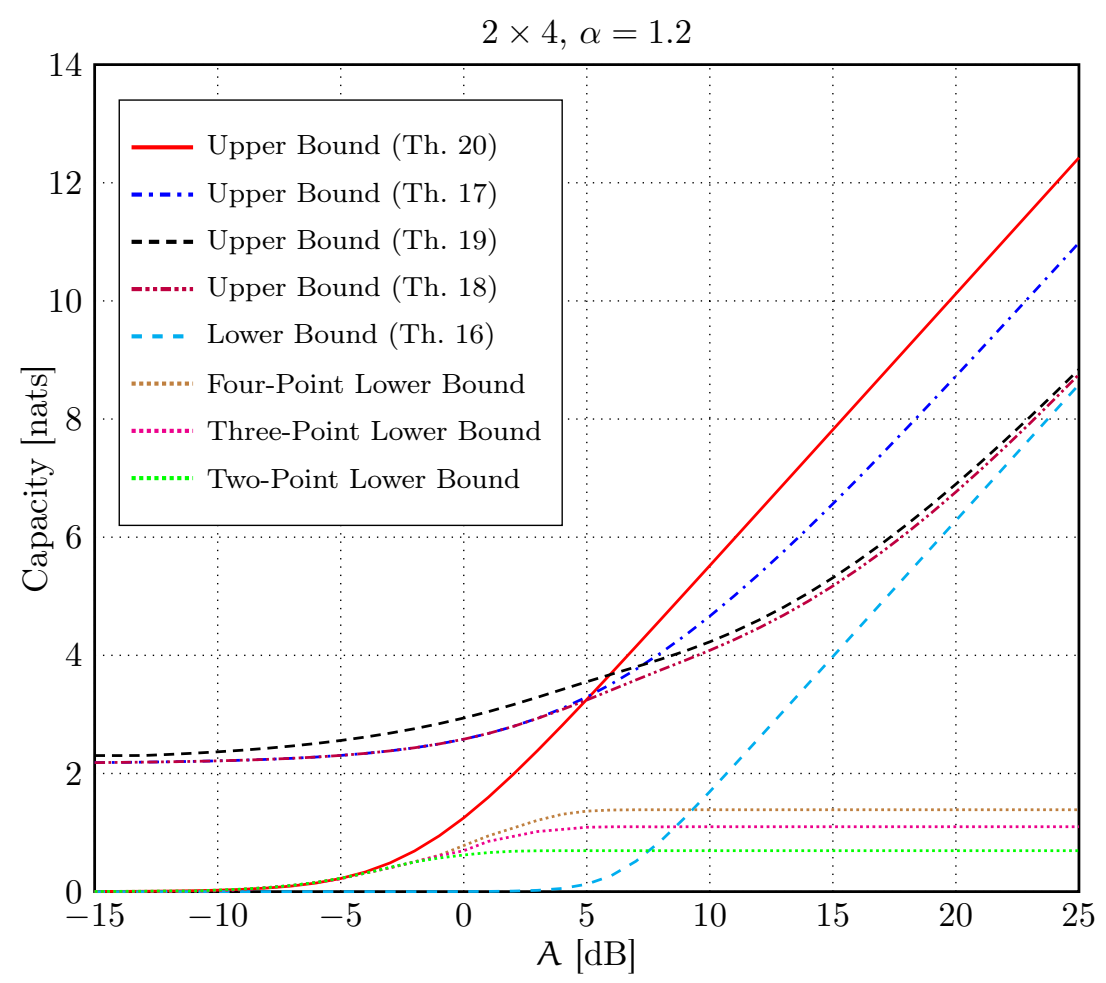

Figure 11: Bounds on capacity of $2 \times 4$ MIMO channel with channel matrix $\mathrm{H}=$ $[1.5,1,0.75,0.5 ; 0.5,0.75,1,1.5]$, and average-to-peak power ratio $\alpha=1.2$. Note that the threshold of the channel is $\alpha_{\mathrm{th}}=1.947$.

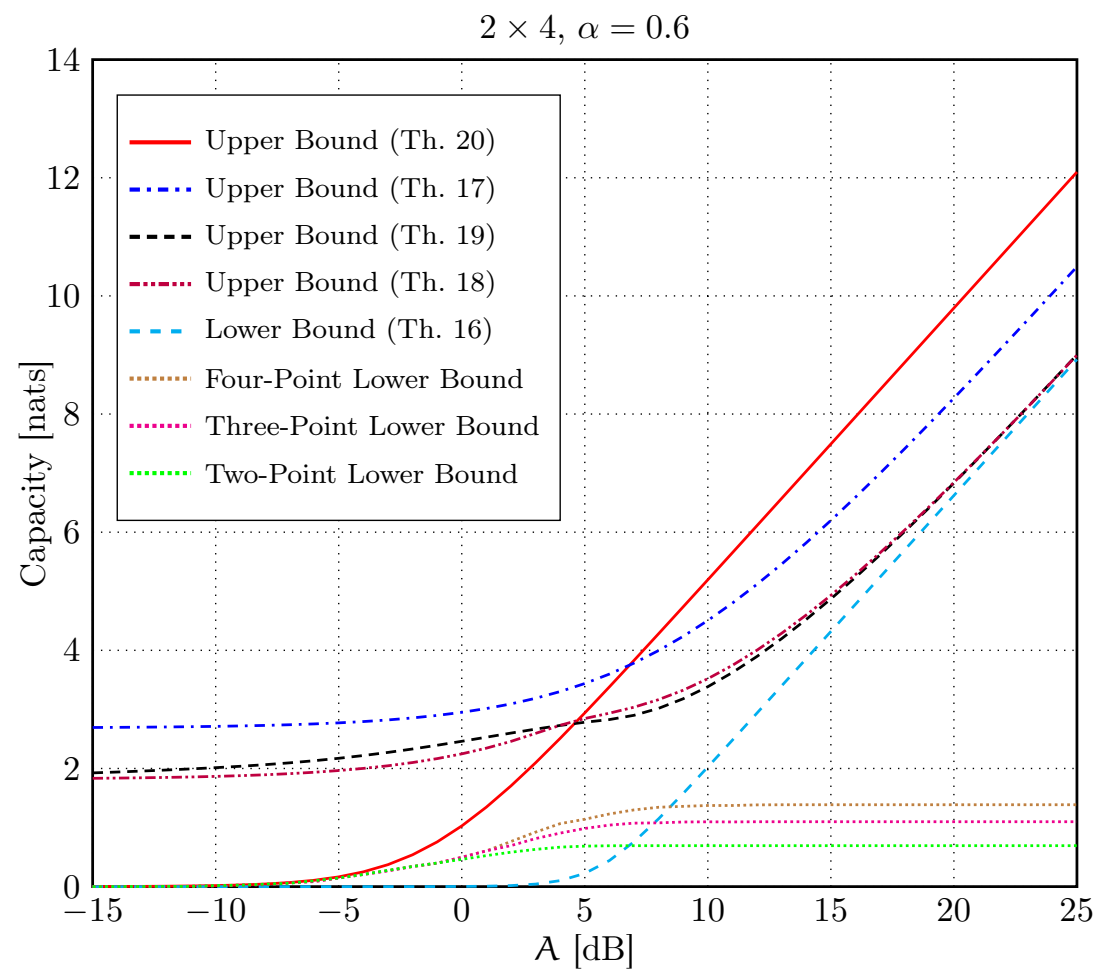

Figure 12: Bounds on capacity of the same $2 \times 4$ MIMO channel as discussed in Figure 11, and average-to-peak power ratio $\alpha=0.6$. 
can then apply previous results on the capacity of the MIMO channel with full-column rank. The remaining steps in deriving our results on the high-SNR asymptotic capacity can be understood, on a high level, as optimizing probabilities and energy constraints assigned to each of the parallel channels.

In the low-SNR regime, the capacity slope is shown to be proportional to the trace of the covariance matrix of $\overline{\mathbf{X}}$ under the given power constraints. We prove several properties of the input distribution that maximizes this trace. For example, each entry in $\mathbf{X}$ should be either zero or the maximum value $A$, and the total number of values of $\mathbf{X}$ with nonzero probabilities need not exceed $n_{\mathrm{R}}+2$.

We recall that, although for IM-DD optical channels, the channel matrix $\mathrm{H}$ typically only has nonnegative entries, our results are valid for all real-valued $\mathrm{H}$.

\section{Acknowledgment}

The authors thank Saïd Ladjal for pointing them to the notion of zonotopes and related literature. They are also grateful for the helpful comments of the Associate Editor and the reviewers.

\section{A Reduction of the Cases $n_{\mathrm{T}}>n_{\mathrm{R}}>\operatorname{rank}(\mathrm{H})$ and $\operatorname{rank}(\mathrm{H}) \leq n_{\mathrm{T}} \leq n_{\mathrm{R}}$}

We first show that a channel with $n_{\mathrm{T}}>n_{\mathrm{R}}>\operatorname{rank}(\mathrm{H})$ can be reduced to one where the rank of the channel matrix equals the number of receive antennas, to which our results apply. Denote

$$
r \triangleq \operatorname{rank}(\mathrm{H})
$$

We apply the SVD

$$
\mathrm{H}=\mathrm{U} \Sigma \mathrm{V}^{\top},
$$

where $\mathrm{U} \in \mathfrak{R}^{n_{\mathrm{R}} \times n_{\mathrm{R}}}$ and $\mathrm{V} \in \mathfrak{R}^{n_{\mathrm{T}} \times n_{\mathrm{T}}}$ are unitary matrices, and $\Sigma \in \mathfrak{R}^{n_{\mathrm{R}} \times n_{\mathrm{T}}}$ is a rectangular matrix whose first $r$ diagonal entries are positive real values, and whose all other entries are zero.

The receiver can compute the new output

$$
\tilde{\mathbf{Y}} \triangleq \mathrm{U}^{\top} \mathbf{Y}=\Sigma \mathrm{V}^{\top} \mathbf{X}+\mathrm{U}^{\top} \mathbf{Z}
$$

where the new noise vector $\tilde{\mathbf{Z}} \triangleq \mathrm{U}^{\top} \mathbf{Z}$ again has independent zero-mean Gaussian components because $\mathrm{U}^{\top}$ is unitary. Moreover, the new channel matrix $\tilde{\mathrm{H}} \triangleq \Sigma \mathrm{V}^{\top}$ is of the form

$$
\tilde{\mathrm{H}}=\left(\begin{array}{l}
\hat{\mathrm{H}} \\
0
\end{array}\right)
$$

with $\hat{\mathrm{H}}$ being an $r \times n_{\mathrm{T}}$ matrix of rank $r$. Combined with the independence of the new noise components, this implies that the outputs $\tilde{Y}_{r+1}, \tilde{Y}_{r+2}, \ldots, \tilde{Y}_{n_{\mathrm{R}}}$ are independent of the first $r$ outputs $\tilde{Y}_{1}, \ldots, \tilde{Y}_{r}$ and the input vector $\mathbf{X}$, and hence can be discarded. The receiver is thus left with outputs $\tilde{Y}_{1}, \ldots, \tilde{Y}_{r}$, in which case the number of receive antennas and the rank of the new channel matrix $\hat{\mathrm{H}}$ both equal $r$. Finally, since we did not change the transmitter side, the inequality $n_{\mathrm{T}}>r$ remains to hold.

The situation where $\operatorname{rank}(\mathrm{H}) \leq n_{\mathrm{T}} \leq n_{\mathrm{R}}$ is handled in the same way, with the only difference that $\tilde{\mathrm{H}}$ in (92) now is tall instead of wide. If $r=n_{\mathrm{T}}, \hat{\mathrm{H}}$ is a full-rank square $n_{\mathrm{T}} \times n_{\mathrm{T}}$ matrix, resulting in a channel model that is not considered in this work, but 
that has been studied extensively in the literature (e.g., [16], [18]). If $r<n_{\mathrm{T}}$, we have again obtained a model, to which our results apply.

Be aware that even if $\mathrm{H}$ only has positive entries, $\hat{\mathrm{H}}$ in (92) may contain negative values.

Note that it is even possible to allow a channel model with dependent noise: Let $\mathrm{K}$ be some positive definite matrix and assume that $\mathbf{Z} \sim \mathcal{N}(\mathbf{0}, \mathrm{K})$. Since $\mathrm{K}$ is positive definite, it can be written as $\mathrm{K}=\mathrm{S}^{\top} \mathrm{S}$ for some invertible $n_{\mathrm{R}} \times n_{\mathrm{R}}$ matrix $\mathrm{S}$. Thus,

$$
\begin{aligned}
\mathrm{I}(\mathbf{X} ; \mathrm{H} \mathbf{X}+\mathbf{Z}) & =\mathrm{I}\left(\mathbf{X} ; \mathrm{S}^{-\mathrm{T}} \mathrm{H} \mathbf{X}+\mathrm{S}^{-\mathrm{T}} \mathbf{Z}\right) \\
& =\mathrm{I}\left(\mathbf{X} ; \mathrm{H}^{\prime} \mathbf{X}+\mathbf{Z}^{\prime}\right),
\end{aligned}
$$

where we define $\mathbf{H}^{\prime} \triangleq \mathrm{S}^{-\mathrm{T}} \mathbf{H}$ and $\mathbf{Z}^{\prime} \triangleq \mathrm{S}^{-\mathrm{T}} \mathbf{Z}$. Note that $\operatorname{rank}\left(\mathrm{H}^{\prime}\right)=\operatorname{rank}(\mathrm{H})$ and that $\mathbf{Z}^{\prime} \sim \mathcal{N}\left(\mathbf{0}, \mathrm{I}_{n_{\mathrm{R}}}\right)$. Therefore, we may apply the same approach as in (90)-(92) to reduce these cases to an equivalent model that is either square full-rank or matches the assumptions (8) considered in this work.

\section{B Proof of Proposition 1}

Fix a capacity-achieving input $\mathbf{X}^{\star}$ and let

$$
\alpha^{*} \triangleq \frac{\mathrm{E}\left[\left\|\mathbf{X}^{\star}\right\|_{1}\right]}{A} .
$$

Define $\mathbf{a} \triangleq(A, A, \ldots, A)^{\top}$ and

$$
\mathbf{X}^{\prime} \triangleq \mathbf{a}-\mathbf{X}^{\star}
$$

We have

$$
\mathrm{E}\left[\left\|\mathbf{X}^{\prime}\right\|_{1}\right]=\mathrm{A}\left(n_{\mathrm{T}}-\alpha^{*}\right),
$$

and

$$
\begin{aligned}
\mathrm{I}\left(\mathbf{X}^{\star} ; \mathbf{Y}\right) & =\mathrm{I}\left(\mathbf{X}^{\star} ; \mathrm{H} \mathbf{a}-\mathbf{Y}\right) \\
& =\mathrm{I}\left(\mathbf{X}^{\star} ; \mathrm{H} \mathbf{a}-\mathrm{H} \mathbf{X}^{\star}-\mathbf{Z}\right) \\
& =\mathrm{I}\left(\mathbf{a}-\mathbf{X}^{\star} ; \mathrm{H}\left(\mathbf{a}-\mathbf{X}^{\star}\right)-\mathbf{Z}\right) \\
& =\mathrm{I}\left(\mathbf{a}-\mathbf{X}^{\star} ; \mathrm{H}\left(\mathbf{a}-\mathbf{X}^{\star}\right)+\mathbf{Z}\right) \\
& =\mathrm{I}\left(\mathbf{X}^{\prime} ; H \mathbf{X}^{\prime}+\mathbf{Z}\right) \\
& =\mathrm{I}\left(\mathbf{X}^{\prime} ; \mathbf{Y}^{\prime}\right)
\end{aligned}
$$

where $\mathbf{Y}^{\prime} \triangleq \mathbf{H X}^{\prime}+\mathbf{Z}$, and where (101) follows because $\mathbf{Z}$ is symmetric around $\mathbf{0}$ and independent of $\mathbf{X}^{\star}$.

Define another random vector $\tilde{\mathbf{X}}$ as follows:

$$
\tilde{\mathbf{X}} \triangleq \begin{cases}\mathbf{X}^{\star} & \text { with probability } p, \\ \mathbf{X}^{\prime} & \text { with probability } 1-p .\end{cases}
$$

Notice that, since $\mathrm{I}(\mathbf{X} ; \mathbf{Y})$ is concave in $P_{\mathbf{X}}$ for a fixed channel law, we have

$$
\mathrm{I}(\tilde{\mathbf{X}} ; \tilde{\mathbf{Y}}) \geq p \mathrm{I}\left(\mathbf{X}^{\star} ; \mathbf{Y}\right)+(1-p) \mathrm{I}\left(\mathbf{X}^{\prime} ; \mathbf{Y}^{\prime}\right) .
$$

Therefore, by (103),

$$
\mathrm{I}(\tilde{\mathbf{X}} ; \tilde{\mathbf{Y}}) \geq \mathrm{I}\left(\mathbf{X}^{\star} ; \mathbf{Y}\right)
$$

for all $p \in[0,1]$. Combined with the assumption that $\mathbf{X}^{\star}$ achieves capacity, (106) implies that $\tilde{\mathbf{X}}$ must also achieve capacity. 
We are now ready to prove the two claims in the proposition. We first prove that for $\alpha>\frac{n_{\mathrm{T}}}{2}$ the average-power constraint is inactive. To this end, we choose $p=\frac{1}{2}$, which yields

$$
\mathrm{E}\left[\|\tilde{\mathbf{X}}\|_{1}\right]=\frac{n_{\mathrm{T}}}{2} \mathrm{~A}
$$

Since $\tilde{\mathbf{X}}$ achieves capacity (see above), we conclude that capacity is unchanged if one strengthens the average-power constraint from $\alpha A$ to $\frac{n_{\mathrm{T}}}{2} \mathrm{~A}$.

We now prove that, if $\alpha \leq \frac{n_{\mathrm{T}}}{2}$, then there exists a capacity-achieving input distribution for which the average-power constraint is met with equality. Assume that $\alpha^{*}<\alpha$ (otherwise $\mathbf{X}^{\star}$ is itself such an input), then choose

$$
p=\frac{n_{\mathrm{T}}-\alpha^{*}-\alpha}{n_{\mathrm{T}}-2 \alpha^{*}} .
$$

With this choice,

$$
\begin{aligned}
\mathrm{E}\left[\|\tilde{\mathbf{X}}\|_{1}\right] & =p \mathrm{E}\left[\left\|\mathbf{X}^{\star}\right\|_{1}\right]+(1-p) \mathrm{E}\left[\left\|\mathbf{X}^{\prime}\right\|_{1}\right] \\
& =\left(p \alpha^{*}+(1-p)\left(n_{\mathrm{T}}-\alpha^{*}\right)\right) \mathrm{A} \\
& =\alpha \mathrm{A} .
\end{aligned}
$$

Hence $\tilde{\mathbf{X}}$ (which achieves capacity) meets the average-power constraint with equality.

\section{Proof of Lemma 5}

First consider the case where Condition (34) is satisfied. Define for each $\mathcal{U} \in \mathscr{U}$ the set

$$
\mathcal{B}_{\mathcal{U}} \triangleq\left\{\mathbf{x}=\left(x_{1}, \ldots, x_{n_{\mathrm{T}}}\right): x_{i} \in(0, \AA), \forall i \in \mathcal{U}, \text { and } x_{j}=A \cdot g_{\mathcal{U}, j}, \forall j \in \mathcal{U}^{\mathrm{c}}\right\}
$$

where we notice that the interval $(0, A)$ is open. We first observe that the optimization problem

$$
\min _{\mathbf{x}^{\prime} \in \mathcal{S}(\overline{\mathbf{x}})}\left\|\mathbf{x}^{\prime}\right\|_{1}
$$

has a solution for every $\overline{\mathbf{x}} \in \mathcal{R}(\mathrm{H})$. This is because the minimization is over a compact set and the objective function is convex and continuous. Furthermore, we have the following lemma.

Lemma 26. Under Condition (34), for all $\overline{\mathbf{x}} \in \mathcal{R}(\mathrm{H})$ except a subset of Lebesgue measure zero, the solutions to (113) lie in the union $\bigcup_{\mathcal{U} \in \mathscr{U}} \mathcal{B}_{\mathcal{U}}$.

Proof: Assume $\mathbf{x}^{\star}=\left(x_{1}^{\star}, \ldots, x_{n_{\mathrm{T}}}^{\star}\right)$ is a solution to (113). It must satisfy the Karush-Kuhn-Tucker (KKT) conditions:

$$
\begin{aligned}
\mathbf{1}_{n_{\mathrm{T}}}-\boldsymbol{\mu}^{\star}+\boldsymbol{\nu}^{\star}+\mathrm{H}^{\top} \boldsymbol{\lambda}^{\star} & =\mathbf{0}_{n_{\mathrm{R}}}, \\
\mathrm{H} \mathbf{x}^{\star}-\overline{\mathbf{x}} & =\mathbf{0}_{n_{\mathrm{R}}}, \\
-\mathbf{x}^{\star} & \leq \mathbf{0}_{n_{\mathrm{T}}}, \\
\mathbf{x}^{\star}-\mathrm{A} \cdot \mathbf{1}_{n_{\mathrm{T}}} & \leq \mathbf{0}_{n_{\mathrm{T}}}, \\
\mu_{i}^{\star} x_{i}^{\star} & =0, \quad i \in\left\{1, \ldots, n_{\mathrm{T}}\right\}, \\
\nu_{i}^{\star}\left(x_{i}^{\star}-\mathrm{A}\right) & =0, \quad i \in\left\{1, \ldots, n_{\mathrm{T}}\right\},
\end{aligned}
$$

for some Lagrange multipliers $\boldsymbol{\mu}^{\star}=\left(\mu_{1}^{\star}, \ldots, \mu_{n_{\mathrm{T}}}^{\star}\right), \boldsymbol{\nu}^{\star}=\left(\nu_{1}^{\star}, \ldots, \nu_{n_{\mathrm{T}}}^{\star}\right)$, and $\boldsymbol{\lambda}^{\star}=$ $\left(\lambda_{1}^{\star}, \ldots, \lambda_{n_{\mathrm{R}}}^{\star}\right)$ satisfying

$$
\boldsymbol{\mu}^{\star} \geq \mathbf{0}_{n_{\mathrm{T}}}
$$




$$
\boldsymbol{\nu}^{\star} \geq \mathbf{0}_{n_{\mathrm{T}}}
$$

All vector inequalities above are componentwise. Let $\mathcal{V}$ be the set of indices corresponding to the components of $\mathbf{x}^{\star}$ in $(0, A)$ :

$$
\mathcal{V} \triangleq\left\{i \in\left\{1, \ldots, n_{\mathrm{T}}\right\}: x_{i}^{\star} \in(0, \mathrm{~A})\right\},
$$

and define $\mathbf{H}_{\mathcal{V}} \triangleq\left[\mathbf{h}_{i}: i \in \mathcal{V}\right]$. Notice that the set of all image vectors that correspond to $\operatorname{rank}\left(\mathrm{H}_{\mathcal{V}}\right)<n_{\mathrm{R}}$ has Lebesgue measure zero. Thus, for the purpose of this proof, we can ignore those image vectors and assume

$$
\operatorname{rank}\left(\mathrm{H}_{\mathcal{V}}\right)=n_{\mathrm{R}}
$$

Clearly, there must exist some $\mathcal{U} \in \mathscr{U}$ such that $\mathcal{U} \subseteq \mathcal{V}$. We next show that $\mathcal{V}=\mathcal{U}$ and therefore $\mathcal{V} \in \mathscr{U}$. We show this by contradiction. Assume there exists an index $i \in \mathcal{V} \backslash \mathcal{U}$. By $(114 \mathrm{e})$ and (114f),

$$
\mu_{j}^{\star}=\nu_{j}^{\star}=0, \quad \forall j \in \mathcal{V}
$$

and thus by (114a),

$$
\left[\mathrm{H}_{\mathcal{U}}, \mathbf{h}_{i}\right]^{\top} \boldsymbol{\lambda}^{\star}=-\mathbf{1}_{n_{\mathrm{R}}+1} .
$$

Since $\mathcal{U} \in \mathscr{U}$, we know that $\mathrm{H}_{\mathcal{U}}$ is invertible, hence

$$
\lambda^{\star}=-\mathrm{H}_{\mathcal{U}}^{-\mathrm{T}} \mathbf{1}_{n_{\mathrm{R}}} .
$$

Plugging this back into (120) yields

$$
\mathbf{h}_{i}^{\top} \mathrm{H}_{\mathcal{U}}^{-\top} \mathbf{1}_{n_{\mathrm{R}}}=1,
$$

which contradicts Condition (34). We hence conclude that $\mathcal{V}=\mathcal{U} \in \mathscr{U}$.

It remains to show that $x_{j}^{\star}=A \cdot g_{\mathcal{U}, j}$ for all $j \in \mathcal{U}^{\mathrm{c}}$. Fix $j \in \mathcal{U}^{\mathrm{c}}$. It follows from (114e) and (114f) that either $\mu_{j}=0$ and $x_{j}^{\star}=A$, or $\nu_{j}=0$ and $x_{j}^{\star}=0$. To determine between these two cases, consider the $j$-th line of (114a):

$$
1-\mu_{j}^{\star}+\nu_{j}^{\star}+\mathbf{h}_{j} \boldsymbol{\lambda}^{\star}=0 .
$$

Note that $\boldsymbol{\lambda}^{\star}$ is given by $(121)$, so

$$
\mathbf{h}_{j}^{\top} \boldsymbol{\lambda}^{\star}=-\mathbf{h}_{j}^{\top} \mathrm{H}_{\mathcal{U}}^{-\top} \mathbf{1}_{n_{\mathrm{R}}}=-a_{\mathcal{U}, j},
$$

hence (123) becomes

$$
-\mu_{j}^{\star}+\nu_{j}^{\star}=a_{\mathcal{U}, j}-1 .
$$

Since both $\mu_{j}^{\star}$ and $\nu_{j}^{\star}$ are nonnegative and only one of them can be positive, we conclude that, when $a_{\mathcal{U}, j}>1$, we must have $\mu_{j}^{\star}=0$ and $x_{j}^{\star}=A$, and when $a_{\mathcal{U}, j}<1$, we must have $\nu_{j}^{\star}=0$ and $x_{j}^{\star}=0$.

We now proceed to prove Lemma 5 for the case where Condition (34) is satisfied. Notice that the $\mathcal{L}_{1}$-norm is continuous, and the correspondence from $\overline{\mathbf{x}}$ to $\mathcal{S}(\overline{\mathbf{x}})$ is compact valued and both lower and upper hemicontinuous. Berge's Maximum Theorem $[29]$ then implies that the correspondence from $\overline{\mathbf{x}}$ to the solutions to (113) is nonempty, compact valued, and upper hemicontinuous. As a consequence, the solutions to (113) for all $\overline{\mathbf{x}} \in \mathcal{R}(\mathrm{H})$ are contained in the closure $\operatorname{cl}\left(\bigcup_{\mathcal{U} \in \mathscr{U}} \mathcal{B}_{\mathcal{U}}\right)$. Because $\mathscr{U}$ is finite, we further have $\operatorname{cl}\left(\bigcup_{\mathcal{U} \in \mathscr{U}} \mathcal{B}_{\mathcal{U}}\right)=\bigcup_{\mathcal{U} \in \mathscr{U}} \operatorname{cl}\left(\mathcal{B}_{\mathcal{U}}\right)$. Thus

$$
\mathcal{R}(\mathrm{H})=\left\{\mathrm{H} \mathbf{x}: \mathrm{x} \in \bigcup_{\mathcal{U} \in \mathscr{U}} \operatorname{cl}\left(\mathcal{B}_{\mathcal{U}}\right)\right\} .
$$


On the other hand, for each $\mathcal{U} \in \mathscr{U}$, we have

$$
\mathbf{v}_{\mathcal{U}}+\mathcal{D}_{\mathcal{U}}=\left\{\mathrm{H} \mathbf{x}: \mathbf{x} \in \operatorname{cl}\left(\mathcal{B}_{\mathcal{U}}\right)\right\} .
$$

Combining (126) and (127) we obtain (40).

Furthermore, because

$$
\operatorname{vol}\left(\mathbf{v}_{\mathcal{U}}+\mathcal{D}_{\mathcal{U}}\right)=A^{n_{\mathrm{R}}}\left|\operatorname{det} \mathrm{H}_{\mathcal{U}}\right|, \quad \forall \mathcal{U} \in \mathscr{U},
$$

and [30], [31]

$$
\operatorname{vol}(\mathcal{R}(\mathrm{H}))=A^{n_{\mathrm{R}}} \sum_{\mathcal{U} \in \mathscr{U}}\left|\operatorname{det} \mathrm{H}_{\mathcal{U}}\right|
$$

we have

$$
\sum_{\mathcal{U} \in \mathscr{U}} \operatorname{vol}\left(\mathbf{v}_{\mathcal{U}}+\mathcal{D}_{\mathcal{U}}\right)=\operatorname{vol}(\mathcal{R}(\mathrm{H})) .
$$

Combined with (40), this proves (41) and completes the first part of the lemma.

We next prove the second part of the lemma. By (41), for any $\mathcal{U}, \mathcal{V} \in \mathscr{U}, \mathcal{U} \neq \mathcal{V}$,

$$
\operatorname{int}\left(\mathbf{v}_{\mathcal{U}}+\mathcal{D}_{\mathcal{U}}\right) \cap \operatorname{int}\left(\mathbf{v}_{\mathcal{V}}+\mathcal{D}_{\mathcal{V}}\right)=\emptyset
$$

where int( $\cdot)$ denotes the interior of a set. Since disjoint open sets are also separated, and since $\operatorname{cl}\left(\operatorname{int}\left(\mathbf{v}_{\mathcal{V}}+\mathcal{D}_{\mathcal{V}}\right)\right)=\mathbf{v}_{\mathcal{V}}+\mathcal{D}_{\mathcal{V}}$, we further obtain

$$
\operatorname{int}\left(\mathbf{v}_{\mathcal{U}}+\mathcal{D}_{\mathcal{U}}\right) \cap\left(\mathbf{v}_{\mathcal{V}}+\mathcal{D}_{\mathcal{V}}\right)=\emptyset
$$

Combined with Lemma 26 and (127), this implies that, for any $\overline{\mathbf{x}} \in \operatorname{int}\left(\mathbf{v}_{\mathcal{U}}+\mathcal{D}_{\mathcal{U}}\right)$, a solution to (113) must lie in $\operatorname{cl}\left(\mathcal{B}_{\mathcal{U}}\right)$ and not in $\operatorname{cl}\left(\mathcal{B}_{\mathcal{V}}\right)$ for any $\mathcal{V} \neq \mathcal{U}$. Then it is immediate that the solution is unique and given by $(42)$. It remains only to extend (42) to the boundaries of $\left\{\mathbf{v}_{\mathcal{U}}+\mathcal{D}_{\mathcal{U}}\right\}_{\mathcal{U} \in \mathscr{U}}$. This is accomplished by recalling that the correspondence from $\overline{\mathbf{x}}$ to the solutions to (113) is upper hemicontinuous. (One can easily verify that, for some $\overline{\mathbf{x}} \in\left(\mathbf{v}_{\mathcal{U}}+\mathcal{D}_{\mathcal{U}}\right) \cap\left(\mathbf{v}_{\mathcal{V}}+\mathcal{D}_{\mathcal{V}}\right)$, computing (42) for $\mathcal{U}$ and $\mathcal{V}$ yields the same result.) This concludes the proof of the second part of Lemma 5.

Finally, we argue that Lemma 5 holds also when (34) is violated. Note that if $a_{\mathcal{U}, j}=1$ for some $\mathcal{U}$ and $j$, then the solution to (113) is not necessarily unique anymore. To solve this problem, note that Algorithm 4 can be interpreted as generating a small perturbation of the matrix $\mathrm{H}$. We fix some small values $\epsilon_{1}>\cdots>\epsilon_{n_{\mathrm{T}}}>0$ and check through all $a_{\mathcal{U}, j}, j \in\left\{1, \ldots, n_{\mathrm{T}}\right\}$. When we encounter a first tie $a_{\mathcal{U}, j}=1$, we multiply the corresponding vector $\mathbf{h}_{j}$ by a factor $\left(1+\epsilon_{1}\right)$ and thereby break the tie $\left(\epsilon_{1}\right.$ is chosen to be small enough so that it does not affect any other choices). If a second tie shows up, we use the next perturbation factor $\left(1+\epsilon_{2}\right)$ (which is smaller than $\left(1+\epsilon_{1}\right)$, so we do not inadvertently revert our first perturbation); and so on. Lemma 5 is then proven by letting all of $\epsilon_{1}, \ldots, \epsilon_{n_{\mathrm{T}}}$ go to zero and invoking Berge's Maximum Theorem. We omit the details.

\section{Proof of Maximum-Variance Signaling Results}

\section{D.1 Proof of Lemma 10}

The $i$ th diagonal element of $\mathrm{K}_{\overline{\mathbf{X}} \overline{\mathbf{x}}}$ can be decomposed as follows:

$$
\left(\mathrm{K}_{\overline{\mathbf{X}} \overline{\mathbf{X}}}\right)_{i, i}=\mathrm{E}\left[\left(\bar{X}_{i}-\mathrm{E}\left[\bar{X}_{i}\right]\right)^{2}\right]
$$




$$
\begin{aligned}
& =\mathrm{E}\left[\left(\sum_{k=1}^{n_{\mathrm{T}}} h_{i, k}\left(X_{k}-\mathrm{E}\left[X_{k}\right]\right)\right)^{2}\right] \\
& =\sum_{k=1}^{n_{\mathrm{T}}} h_{i, k}^{2} \mathrm{E}\left[\left(X_{k}-\mathrm{E}\left[X_{k}\right]\right)^{2}\right]+\sum_{k=1}^{n_{\mathrm{T}}} \sum_{\substack{\ell=1 \\
\ell \neq k}}^{n_{\mathrm{T}}} h_{i, k} h_{i, \ell}\left(\mathrm{E}\left[X_{k} X_{\ell}\right]-\mathrm{E}\left[X_{k}\right] \mathrm{E}\left[X_{\ell}\right]\right) .
\end{aligned}
$$

Thus, the objective function in (56) is

$$
\sum_{i=1}^{n_{\mathrm{R}}} \sum_{k=1}^{n_{\mathrm{T}}} h_{i, k}^{2} \mathrm{E}\left[\left(X_{k}-\mathrm{E}\left[X_{k}\right]\right)^{2}\right]+\sum_{i=1}^{n_{\mathrm{R}}} \sum_{k=1}^{n_{\mathrm{T}}} \sum_{\substack{\ell=1 \\ \ell \neq k}}^{n_{\mathrm{T}}} h_{i, k} h_{i, \ell}\left(\mathrm{E}\left[X_{k} X_{\ell}\right]-\mathrm{E}\left[X_{k}\right] \mathrm{E}\left[X_{\ell}\right]\right) .
$$

If we fix a joint distribution on $\left(X_{1}, \ldots, X_{n_{\mathrm{T}}-1}\right)$ and choose with probability 1 a conditional mean $\mathrm{E}\left[X_{n_{\mathrm{T}}} \mid X_{1}, \ldots, X_{n_{\mathrm{T}}-1}\right]$, then the consumed total average input power is fixed and every summand on the RHS of (136) is determined except for

$$
\mathrm{E}\left[\left(X_{n_{\mathrm{T}}}-\mathrm{E}\left[X_{n_{\mathrm{T}}}\right]\right)^{2}\right] .
$$

This value is maximized - for any choice of joint distribution on $\left(X_{1}, \ldots, X_{n_{\mathrm{T}}-1}\right)$ and conditional mean $\mathrm{E}\left[X_{n_{\mathrm{T}}} \mid X_{1}, \ldots, X_{n_{\mathrm{T}}-1}\right]$ - if $X_{n_{\mathrm{T}}}$ takes value only in the set $\{0, A\}$. We conclude that, to maximize the expression in (56) subject to a constraint on the average input power, it is optimal to restrict $X_{n_{\mathrm{T}}}$ to taking value only in $\{0, A\}$.

Repeating this argument for $X_{n_{\mathrm{T}}-1}, X_{n_{\mathrm{T}}-2}$, etc., we conclude that every $X_{k}, k=$ $1, \ldots, n_{\mathrm{T}}$, should take value only in $\{0, A\}$.

\section{D.2 Proof of Lemma 11}

Some steps in our proof are inspired by [20]. We start by rewriting the objective function in (56) as:

$$
\begin{aligned}
\operatorname{tr}\left(\mathrm{K}_{\overline{\mathbf{X}} \overline{\mathbf{X}}}\right) & =\sum_{i=1}^{n_{\mathrm{R}}} \mathrm{E}\left[\left(\bar{X}_{i}-\mathrm{E}\left[\bar{X}_{i}\right]\right)^{2}\right] \\
& =\sum_{i=1}^{n_{\mathrm{R}}} \mathrm{E}\left[\left(\sum_{k=1}^{n_{\mathrm{T}}} h_{i, k}\left(X_{k}-\mathrm{E}\left[X_{k}\right]\right)\right)^{2}\right] \\
& =\sum_{i=1}^{n_{\mathrm{R}}} \sum_{k=1}^{n_{\mathrm{T}}} \sum_{k^{\prime}=1}^{n_{\mathrm{T}}} h_{i, k} h_{i, k^{\prime}} \mathrm{E}\left[\left(X_{k}-\mathrm{E}\left[X_{k}\right]\right)\left(X_{k^{\prime}}-\mathrm{E}\left[X_{k^{\prime}}\right]\right)\right] \\
& =\sum_{k=1}^{n_{\mathrm{T}}} \sum_{k^{\prime}=1}^{n_{\mathrm{T}}} \underbrace{\operatorname{Cov}\left[X_{k}, X_{k^{\prime}}\right]}_{\sum_{i=1}^{n_{\mathrm{R}}} h_{i, k} h_{i, k^{\prime}}} \\
& =\sum_{k=1}^{n_{\mathrm{T}}} \sum_{k^{\prime}=1}^{n_{\mathrm{T}}} \kappa_{k, k^{\prime}} \operatorname{Cov}\left[X_{k}, X_{k^{\prime}}\right] .
\end{aligned}
$$

Thus, we need to maximize $\operatorname{Cov}\left[X_{k}, X_{k^{\prime}}\right]$. Assume that we have fixed the average power $\mathrm{E}_{k}, k=1, \ldots, n_{\mathrm{T}}$, assigned to each input antenna, and further assume that we reorder the antennas such that

$$
\mathrm{E}_{1} \geq \cdots \geq \mathrm{E}_{n_{\mathrm{T}}} .
$$

Note that since each antenna only uses a binary input $X_{k} \in\{0, A\}$, the assignment $\mathrm{E}\left[X_{k}\right]=\mathrm{E}_{k}$ determines the probabilities:

$$
\operatorname{Pr}\left[X_{k}=\mathrm{A}\right]=\frac{\mathrm{E}_{k}}{\mathrm{~A}}
$$


and the variances:

$$
\operatorname{Cov}\left[X_{k}, X_{k}\right]=\operatorname{Var}\left[X_{k}\right]=\mathrm{E}\left[X_{k}^{2}\right]-\mathrm{E}_{k}^{2}=\mathrm{E}_{k} \mathrm{~A}-\mathrm{E}_{k}^{2} .
$$

For the covariances with $k<k^{\prime}$ we obtain

$$
\begin{aligned}
\operatorname{Cov}\left[X_{k}, X_{k^{\prime}}\right] & =\mathrm{E}\left[X_{k} X_{k^{\prime}}\right]-\mathrm{E}_{k} \mathrm{E}_{k^{\prime}} \\
& =A^{2} \operatorname{Pr}\left[X_{k}=X_{k^{\prime}}=\mathrm{A}\right]-\mathrm{E}_{k} \mathrm{E}_{k^{\prime}} \\
& =A^{2} \operatorname{Pr}\left[X_{k^{\prime}}=\mathrm{A}\right] \underbrace{\operatorname{Pr}\left[X_{k}=A \mid X_{k^{\prime}}=A\right]}_{\leq 1}-\mathrm{E}_{k} \mathrm{E}_{k^{\prime}} \\
& \leq A \mathrm{E}_{k^{\prime}}-\mathrm{E}_{k} \mathrm{E}_{k^{\prime}} \\
& =\left(A-\mathrm{E}_{k}\right) \mathrm{E}_{k^{\prime}} .
\end{aligned}
$$

The upper bound holds with equality if

$$
\operatorname{Pr}\left[X_{k}=\mathrm{A} \mid X_{k^{\prime}}=\mathrm{A}\right]=1 .
$$

This choice is allowed, because for $k<k^{\prime}$ the ordering (143) is compatible with Condition (151). This proves that the mass points can be ordered in such a way that (58) holds.

We next prove by contradiction that the first mass point must be $\mathbf{0}$. By Lemma 10, if $\mathbf{x}_{1}^{*} \neq \mathbf{0}$, then $\mathbf{x}_{1}^{*}$ must contain at least one entry that equals $A$. By (58), that entry must be $A$ for all mass points used by the optimal input. Clearly, changing its value from $A$ to 0 for all mass points will not affect the trace of (55), but will reduce the total input power. Hence we conclude that an input with $\mathbf{x}_{1}^{*} \neq \mathbf{0}$ (or with zero probability on 0) must be suboptimal.

\section{D.3 Proof of Lemma 12}

We investigate the KKT conditions of the optimization problem (56). Using the definition of $\mathscr{T}$ and $r_{\mathcal{V}, i}$ we rewrite the objective function of (56) as

$$
\begin{aligned}
\operatorname{tr}\left(\mathrm{K}_{\overline{\mathbf{X}} \overline{\mathbf{X}}}\right) & =\sum_{i=1}^{n_{\mathrm{R}}}\left(\mathrm{E}\left[\bar{X}_{i}^{2}\right]-\left(\mathrm{E}\left[\bar{X}_{i}\right]\right)^{2}\right) \\
& =A^{2} \sum_{i=1}^{n_{\mathrm{R}}}\left(\sum_{\mathcal{V} \in \mathscr{T}} p_{\mathcal{V}} r_{\mathcal{V}, i}^{2}-\left(\sum_{\mathcal{V} \in \mathscr{T}} p_{\mathcal{V}} r_{\mathcal{V}, i}\right)^{2}\right)
\end{aligned}
$$

Taking into account the constraints (6), the Lagrangian is obtained as:

$$
\begin{aligned}
\mathrm{L}\left(\mathbf{p}, \mu_{0}, \mu_{1}, \boldsymbol{\mu}\right)= & A^{2} \sum_{i=1}^{n_{\mathrm{R}}}\left(\sum_{\mathcal{V} \in \mathscr{T}} p_{\mathcal{V}} r_{\mathcal{V}, i}^{2}-\left(\sum_{\mathcal{V} \in \mathscr{T}} p_{\mathcal{V}} r_{\mathcal{V}, i}\right)^{2}\right)-\mu_{0}\left(\sum_{\mathcal{V} \in \mathscr{T}} p_{\mathcal{V}}-1\right) \\
& -\mu_{1}\left(\sum_{\mathcal{V} \in \mathscr{T}} p_{\mathcal{V}}|\mathcal{V}|-\alpha\right)-\sum_{\mathcal{V} \in \mathscr{T}} \mu_{\mathcal{V}}\left(0-p_{\mathcal{V}}\right) .
\end{aligned}
$$

The KKT conditions for the optimal $\left\{p_{\mathcal{K}}^{*}\right\}_{\mathcal{K} \in \mathscr{U}}$ are as follows:

$$
\begin{aligned}
A^{2} \sum_{i=1}^{n_{\mathrm{R}}}\left(r_{\mathcal{K}, i}^{2}-2 r_{\mathcal{K}, i} \sum_{\mathcal{V} \in \mathscr{T}} p_{\mathcal{V}}^{*} r_{\mathcal{V}, i}\right)-\mu_{0}-\mu_{1}|\mathcal{K}|+\mu_{\mathcal{K}} & =0, \quad \mathcal{K} \in \mathscr{T}, \\
\mu_{0}\left(\sum_{\mathcal{V} \in \mathscr{T}} p_{\mathcal{V}}^{*}-1\right) & =0, \\
\mu_{1}\left(\sum_{\mathcal{V} \in \mathscr{T}} p_{\mathcal{V}}^{*}|\mathcal{V}|-\alpha\right) & =0,
\end{aligned}
$$




$$
\begin{aligned}
\mu_{\mathcal{K}} p_{\mathcal{K}}^{*} & =0, \quad \mathcal{K} \in \mathscr{T}, \\
\mu_{0} & \geq 0, \\
\mu_{1} & \geq 0, \\
\mu_{\mathcal{K}} & \geq 0, \quad \mathcal{K} \in \mathscr{T}, \\
\sum_{\mathcal{V} \in \mathscr{T}} p_{\mathcal{V}}^{*} & \leq 1, \\
\sum_{\mathcal{V} \in \mathscr{T}} p_{\mathcal{V}}^{*}|\mathcal{V}| & \leq \alpha, \\
p_{\mathcal{K}}^{*} & \geq 0, \quad \mathcal{K} \in \mathscr{T} .
\end{aligned}
$$

We define the vector $\mathbf{m}=\left(m_{1}, \ldots, m_{n_{\mathrm{R}}}\right)^{\mathrm{T}}$ with components

$$
m_{i} \triangleq \sum_{\mathcal{V} \in \mathscr{T}} p_{\mathcal{V}}^{*} r_{\mathcal{V}, i}, \quad i=1, \ldots, n_{\mathrm{R}},
$$

and rewrite (155a) as

$$
A^{2}\left\|\mathbf{r}_{\mathcal{K}}\right\|_{2}^{2}-2 A^{2} \mathbf{r}_{\mathcal{K}}^{\top} \mathbf{m}-\mu_{0}-\mu_{1}|\mathcal{K}|+\mu_{\mathcal{K}}=0, \quad \mathcal{K} \in \mathscr{T} .
$$

Since by Lemma $11 P_{\mathbf{X}}^{*}(\mathbf{0})>0$, it must hold that $(155 \mathrm{~h})$ holds with strict inequality and it thus follows from (155b) that $\mu_{0}=0$.

Next, assume by contradiction that there exist $n_{\mathrm{R}}+2$ choices $\mathcal{K}_{1}, \ldots, \mathcal{K}_{n_{\mathrm{R}}+2} \in \mathscr{T}$ with positive probability $p_{\mathcal{K}_{\ell}}^{*}>0$. Then, by $(155 \mathrm{~d}), \mu_{\mathcal{K}_{\ell}}=0$ for all $\ell \in\left\{1, \ldots, n_{\mathrm{R}}+2\right\}$. From (157) we thus have

$$
2 \mathbf{r}_{\mathcal{K}_{\ell}}^{\top} \mathbf{m}+\tilde{\mu}_{1}\left|\mathcal{K}_{\ell}\right|=\left\|\mathbf{r}_{\mathcal{K}_{\ell}}\right\|_{2}^{2}, \quad \ell \in\left\{1, \ldots, n_{\mathrm{R}}+2\right\},
$$

with $\tilde{\mu}_{1} \triangleq \mu_{1} / A^{2}$, which can be written in matrix form:

$$
\left(\begin{array}{cccc}
2 r_{\mathcal{K}_{1}, 1} & \cdots & 2 r_{\mathcal{K}_{1}, n_{\mathrm{R}}} & \left|\mathcal{K}_{1}\right| \\
2 r_{\mathcal{K}_{2}, 1} & \cdots & 2 r_{\mathcal{K}_{2}, n_{\mathrm{R}}} & \left|\mathcal{K}_{2}\right| \\
\vdots & \ddots & \vdots & \vdots \\
2 r_{\mathcal{K}_{n_{\mathrm{R}}+2}, 1} & \cdots & 2 r_{\mathcal{K}_{n_{\mathrm{R}}+2, n_{\mathrm{R}}}} & \left|\mathcal{K}_{n_{\mathrm{R}}+2}\right|
\end{array}\right)\left(\begin{array}{c}
m_{1} \\
m_{2} \\
\vdots \\
m_{n_{\mathrm{R}}} \\
\tilde{\mu}_{1}
\end{array}\right)=\left(\begin{array}{c}
\left\|\mathbf{r}_{\mathcal{K}_{1}}\right\|_{2}^{2} \\
\left\|\mathbf{r}_{\mathcal{K}_{2}}\right\|_{2}^{2} \\
\vdots \\
\left\|\mathbf{r}_{\mathcal{K}_{n_{\mathrm{R}}+2}}\right\|_{2}^{2}
\end{array}\right) .
$$

This is an over-determined system of linear equations in $n_{\mathrm{R}}+1$ variables $m_{1}, \ldots, m_{n_{\mathrm{R}}}, \tilde{\mu}_{1}$, which has a solution if, and only if,

$$
\begin{aligned}
\operatorname{rank}\left(\begin{array}{ccccc}
2 r_{\mathcal{K}_{1}, 1} & \cdots & 2 r_{\mathcal{K}_{1}, n_{\mathrm{R}}} & \left|\mathcal{K}_{1}\right| \\
2 r_{\mathcal{K}_{2}, 1} & \cdots & 2 r_{\mathcal{K}_{2}, n_{\mathrm{R}}} & \left|\mathcal{K}_{2}\right| \\
\vdots & \ddots & \vdots & \vdots \\
2 r_{\mathcal{K}_{n_{\mathrm{R}}+2}, 1} & \cdots & 2 r_{\mathcal{K}_{n_{\mathrm{R}}+2}, n_{\mathrm{R}}} & \left|\mathcal{K}_{n_{\mathrm{R}}+2}\right|
\end{array}\right) \\
=\operatorname{rank}\left(\begin{array}{ccccc}
2 r_{\mathcal{K}_{1}, 1} & \cdots & 2 r_{\mathcal{K}_{1}, n_{\mathrm{R}}} & \left|\mathcal{K}_{1}\right| & \left\|\mathbf{r}_{\mathcal{K}_{1}}\right\|_{2}^{2} \\
2 r_{\mathcal{K}_{2}, 1} & \cdots & 2 r_{\mathcal{K}_{2}, n_{\mathrm{R}}} & \left|\mathcal{K}_{2}\right| & \left\|\mathbf{r}_{\mathcal{K}_{2}}\right\|_{2}^{2} \\
\vdots & \ddots & \vdots & \vdots & \vdots \\
2 r_{\mathcal{K}_{n_{\mathrm{R}}+2}, 1} & \cdots & 2 r_{\mathcal{K}_{n_{\mathrm{R}}+2}, n_{\mathrm{R}}} & \left|\mathcal{K}_{n_{\mathrm{R}}+2}\right| & \left\|\mathbf{r}_{\mathcal{K}_{n_{\mathrm{R}}+2}}\right\|_{2}^{2}
\end{array}\right) .
\end{aligned}
$$

However, since the matrix on the LHS has only $n_{\mathrm{R}}+1$ columns, its rank can be at most $n_{\mathrm{R}}+1$. The matrix on the RHS, on the other hand, has by assumption (see (63)) rank $n_{\mathrm{R}}+2$. This is a contradiction. We have proven that there exist at most $n_{\mathrm{R}}+1$ values $p_{\mathcal{K}}$ with positive values. Together with $\mathbf{0}$, there are at most $n_{\mathrm{R}}+2$ mass points in total.

\section{E Derivation of the Lower Bounds}

For any choice of the random vector $\overline{\mathbf{X}}$ over $\mathcal{R}(\mathrm{H})$, the following holds:

$$
\mathrm{C}_{\mathrm{H}}(\mathrm{A}, \alpha \mathrm{A}) \geq \mathrm{I}(\overline{\mathbf{X}} ; \overline{\mathbf{X}}+\mathbf{Z})
$$




$$
\begin{aligned}
& =\mathrm{h}(\overline{\mathbf{X}}+\mathbf{Z})-\mathrm{h}(\mathbf{Z}) \\
& \geq \frac{1}{2} \log \left(e^{2 \mathrm{~h}(\overline{\mathbf{X}})}+e^{2 \mathrm{~h}(\mathbf{Z})}\right)-\mathrm{h}(\mathbf{Z}) \\
& =\frac{1}{2} \log \left(1+\frac{e^{2 \mathrm{~h}(\overline{\mathbf{X}})}}{(2 \pi e)^{n_{\mathrm{R}}}}\right),
\end{aligned}
$$

where (163) follows from the EPI [27].

\section{E.1 Proof of Theorem 15}

We choose $\overline{\mathbf{X}}$ to be uniformly distributed over $\mathcal{R}(\mathrm{H})$. To verify that this uniform distribution satisfies the average-power constraint (48), we define

$$
p_{\mathcal{U}} \triangleq \operatorname{Pr}[\mathbb{U}=\mathcal{U}]
$$

and derive

$$
\begin{aligned}
\mathrm{E}_{\mathbb{U}} & {\left[\mathrm{A} s_{\mathbb{U}}+\left\|\mathrm{H}_{\mathbb{U}}^{-1}\left(\mathrm{E}[\overline{\mathbf{X}} \mid \mathbb{U}]-\mathbf{v}_{\mathbb{U}}\right)\right\|_{1}\right] } \\
& =\mathrm{A} \sum_{\mathcal{U} \in \mathscr{U}} p_{\mathcal{U}} s_{\mathcal{U}}+\sum_{\mathcal{U} \in \mathscr{U}} p_{\mathcal{U}}\left\|\mathrm{H}_{\mathcal{U}}^{-1}\left(\mathrm{E}[\overline{\mathbf{X}} \mid \mathbb{U}=\mathcal{U}]-\mathbf{v}_{\mathcal{U}}\right)\right\|_{1} \\
& =\mathrm{A} \sum_{\mathcal{U} \in \mathscr{U}} q_{\mathcal{U}} s_{\mathcal{U}}+\sum_{\mathcal{U} \in \mathscr{U}} q_{\mathcal{U}} \cdot \frac{n_{\mathrm{R}} \mathrm{A}}{2} \\
& =\alpha_{\mathrm{th}} \mathrm{A} \\
& \leq \alpha \mathrm{A}
\end{aligned}
$$

Here, (167) follows because when $\overline{\mathbf{X}}$ is uniformly distributed in $\mathcal{R}(\mathrm{H})$, we have

$$
\mathrm{H}_{\mathcal{U}}^{-1}\left(\mathrm{E}[\overline{\mathbf{X}} \mid \mathbb{U}=\mathcal{U}]-\mathbf{v}_{\mathcal{U}}\right)=\frac{A}{2} \cdot \mathbf{1}_{n_{\mathrm{R}}}
$$

and

$$
p_{\mathcal{U}}=q_{\mathcal{U}}, \quad \mathcal{U} \in \mathscr{U} .
$$

Further, (168) holds because of (67), and the last inequality (169) holds by the assumption in the theorem.

The uniform distribution of $\overline{\mathbf{X}}$ results in

$$
h(\overline{\mathbf{X}})=\log \left(A^{n_{R}} \cdot V_{H}\right)
$$

which, by (164), leads to (75).

\section{E.2 Proof of Theorem 16}

We choose

$$
\lambda \in\left(\max \left\{0, \frac{n_{\mathrm{R}}}{2}+\alpha-\alpha_{\mathrm{th}}\right\}, \min \left\{\frac{n_{\mathrm{R}}}{2}, \alpha\right\}\right),
$$

a probability vector $\mathbf{p}$ satisfying (79), and $\mu$ as the unique solution to (78).

Note that such choices are always possible as can be argued as follows. From (173) one directly sees that $0<\lambda<\frac{n_{\mathrm{R}}}{2}$. Thus, $0<\frac{\lambda}{n_{\mathrm{R}}}<\frac{1}{2}$, which corresponds exactly to the range where (78) has a unique solution. From (173) it also follows that $\frac{n_{\mathrm{R}}}{2}+\alpha-\alpha_{\text {th }}<\lambda<\alpha$ and thus

$$
0<\alpha-\lambda<\alpha_{\mathrm{th}}-\frac{n_{\mathrm{R}}}{2} \leq \frac{n_{\mathrm{T}}}{2}-\frac{n_{\mathrm{R}}}{2},
$$


where the last inequality follows from (71). So the RHS of (79) takes value within the interval $\left(0, \frac{n_{\mathrm{T}}-n_{\mathrm{R}}}{2}\right)$. By Remark 7 , the LHS of (79) can take value in the interval $\left[0, n_{\mathrm{T}}-n_{\mathrm{R}}\right]$, which covers the range of the RHS. The existence of $\mathbf{p}$ satisfying (79) now follows from the continuity of the LHS of (79) in $\mathbf{p}$.

For each $\mathcal{U}$ we now pick the probability density function $f_{\overline{\mathbf{X}} \mid \mathbb{U}=\mathcal{U}}$ to be the $n_{\mathrm{R}}$ dimensional product truncated exponential distribution rotated by the matrix $\mathrm{H}_{\mathcal{U}}$ :

$$
f_{\overline{\mathbf{X}} \mid \mathbb{U}=\mathcal{U}}(\overline{\mathbf{x}})=\frac{1}{A^{n_{\mathrm{R}}}\left|\operatorname{det} \mathrm{H}_{\mathcal{U}}\right|} \cdot\left(\frac{\mu}{1-e^{-\mu}}\right)^{n_{\mathrm{R}}} e^{-\frac{\mu}{A}\left\|\mathbf{H}_{\mathcal{U}}^{-1}(\overline{\mathbf{x}}-\mathbf{v} \mathcal{U})\right\|_{1}} .
$$

Note that this corresponds to the entropy-maximizing distribution under a total averagepower constraint. The average-power constraint (48) is satisfied because

$$
\begin{aligned}
\mathrm{E}_{\mathbb{U}} & {\left[\mathrm{A}_{\mathbb{U}}+\left\|\mathrm{H}_{\mathbb{U}}^{-1}\left(\mathrm{E}[\overline{\mathbf{X}} \mid \mathbb{U}]-\mathbf{v}_{\mathbb{U}}\right)\right\|_{1}\right] } \\
& =\sum_{\mathcal{U} \in \mathscr{U}} p_{\mathcal{U}}\left(\mathrm{A} s_{\mathcal{U}}+\left\|\mathrm{H}_{\mathcal{U}}^{-1}\left(\mathrm{E}[\overline{\mathbf{X}} \mid \mathbb{U}=\mathcal{U}]-\mathbf{v}_{\mathcal{U}}\right)\right\|_{1}\right) \\
& =\sum_{\mathcal{U} \in \mathscr{U}} p_{\mathcal{U}}\left(A s_{\mathcal{U}}+n_{\mathrm{R}} \mathrm{A}\left(\frac{1}{\mu}-\frac{e^{-\mu}}{1-e^{-\mu}}\right)\right) \\
& =\sum_{\mathcal{U} \in \mathscr{U}} p_{\mathcal{U}}\left(\mathrm{A} s_{\mathcal{U}}+\mathrm{A} \lambda\right) \\
& =\mathrm{A} \sum_{\mathcal{U} \in \mathscr{U}} p_{\mathcal{U}} s_{\mathcal{U}}+\mathrm{A} \lambda \\
& =\mathrm{A}(\alpha-\lambda)+\mathrm{A} \lambda \\
& =\alpha A .
\end{aligned}
$$

Here, (177) follows from the expected value of the truncated exponential distribution; (178) is due to (78); and (180) follows from (79).

Furthermore,

$$
\begin{aligned}
\mathrm{h}(\overline{\mathbf{X}})= & \mathrm{I}(\overline{\mathbf{X}} ; \mathbb{U})+\mathrm{h}(\overline{\mathbf{X}} \mid \mathbb{U}) \\
= & \mathrm{H}(\mathbb{U})+\mathrm{h}(\overline{\mathbf{X}} \mid \mathbb{U}) \\
= & \mathrm{H}(\mathbf{p})+\sum_{\mathcal{U} \in \mathscr{U}} p_{\mathcal{U}} \mathrm{h}(\overline{\mathbf{X}} \mid \mathbb{U}=\mathcal{U}) \\
= & \mathrm{H}(\mathbf{p})+\sum_{\mathcal{U} \in \mathscr{U}} p_{\mathcal{U}} \log \left|\operatorname{det} \mathrm{H}_{\mathcal{U}}\right|+n_{\mathrm{R}} \log \mathrm{A}-n_{\mathrm{R}} \log \frac{\mu}{1-e^{-\mu}} \\
& +n_{\mathrm{R}}\left(1-\frac{\mu e^{-\mu}}{1-e^{-\mu}}\right) \\
= & -\sum_{\mathcal{U} \in \mathscr{U}} p_{\mathcal{U}} \log p_{\mathcal{U}}+\sum_{\mathcal{U} \in \mathscr{U}} p_{\mathcal{U}} \log \frac{\left|\operatorname{det} \mathrm{H}_{\mathcal{U}}\right|}{\mathrm{V}_{\mathrm{H}}}+\log \mathrm{V}_{\mathrm{H}}+n_{\mathrm{R}} \log \mathrm{A} \\
& +n_{\mathrm{R}}\left(1-\log \frac{\mu}{1-e^{-\mu}}-\frac{\mu e^{-\mu}}{1-e^{-\mu}}\right) \\
= & -\mathrm{D}(\mathbf{p} \| \mathbf{q})+\log \mathbf{V}_{\mathrm{H}}+n_{\mathrm{R}} \log \mathrm{A}+n_{\mathrm{R}}\left(1-\log \frac{\mu}{1-e^{-\mu}}-\frac{\mu e^{-\mu}}{1-e^{-\mu}}\right) .
\end{aligned}
$$

Here, (183) holds because $\mathrm{H}(\mathbb{U} \mid \overline{\mathbf{X}})=0$; (185) follows from the differential entropy of a truncated exponential distribution; and in (187) we use the definition of $\mathbf{q}$ in (66). Then, (76) follows by plugging (187) into (164).

\section{F Derivation of Upper Bounds}

Let $\overline{\mathbf{X}}^{\star}$ be a maximizer in (47) and let $\mathbb{U}^{\star}$ be defined by $\overline{\mathbf{X}}^{\star}$ as in (46). Then,

$$
\mathrm{C}_{\mathrm{H}}(\mathrm{A}, \alpha \mathrm{A})=\mathrm{I}\left(\overline{\mathbf{X}}^{\star} ; \overline{\mathbf{X}}^{\star}+\mathbf{Z}\right)
$$




$$
\begin{aligned}
& \leq \mathrm{I}\left(\overline{\mathbf{X}}^{\star} ; \overline{\mathbf{X}}^{\star}+\mathbf{Z}, \mathbb{U}^{\star}\right) \\
& \leq \mathrm{H}\left(\mathbb{U}^{\star}\right)+\mathrm{I}\left(\overline{\mathbf{X}}^{\star} ; \overline{\mathbf{X}}^{\star}+\mathbf{Z} \mid \mathbb{U}^{\star}\right) .
\end{aligned}
$$

For each $\operatorname{set} \mathcal{U} \in \mathscr{U}$, we have

$$
\begin{aligned}
\mathrm{I}\left(\overline{\mathbf{X}}^{\star} ; \overline{\mathbf{X}}^{\star}+\mathbf{Z} \mid \mathbb{U}^{\star}=\mathcal{U}\right) & =\mathrm{I}\left(\overline{\mathbf{X}}^{\star}-\mathbf{v}_{\mathcal{U}} ;\left(\overline{\mathbf{X}}^{\star}-\mathbf{v}_{\mathcal{U}}\right)+\mathbf{Z} \mid \mathbb{U}^{\star}=\mathcal{U}\right) \\
& =\mathrm{I}\left(\mathrm{H}_{\mathcal{U}}^{-1}\left(\overline{\mathbf{X}}^{\star}-\mathbf{v}_{\mathcal{U}}\right) ; \mathrm{H}_{\mathcal{U}}^{-1}\left(\overline{\mathbf{X}}^{\star}-\mathbf{v}_{\mathcal{U}}\right)+\mathrm{H}_{\mathcal{U}}^{-1} \mathbf{Z} \mid \mathbb{U}^{\star}=\mathcal{U}\right) \\
& =\mathrm{I}\left(\mathbf{X}_{\mathcal{U}} ; \mathbf{X}_{\mathcal{U}}+\mathbf{Z}_{\mathcal{U}} \mid \mathbb{U}^{\star}=\mathcal{U}\right)
\end{aligned}
$$

where we have defined

$$
\begin{aligned}
\mathbf{Z}_{\mathcal{U}} & \triangleq \mathrm{H}_{\mathcal{U}}^{-1} \mathbf{Z}, \\
\mathbf{X}_{\mathcal{U}} & \triangleq \mathrm{H}_{\mathcal{U}}^{-1}\left(\overline{\mathbf{X}}^{\star}-\mathbf{v}_{\mathcal{U}}\right) .
\end{aligned}
$$

It should be noted that

$$
\mathbf{Z}_{\mathcal{U}} \sim \mathcal{N}\left(0, \mathrm{H}_{\mathcal{U}}^{-1} \mathrm{H}_{\mathcal{U}}^{-\top}\right)
$$

Moreover, $\mathbf{X}_{\mathcal{U}}$ is subject to the following peak- and average-power constraints:

$$
\begin{aligned}
\operatorname{Pr}\left[X_{\mathcal{U}, \ell}>\mathrm{A}\right] & =0, \quad \forall \ell \in\left\{1, \ldots, n_{\mathrm{R}}\right\}, \\
\mathrm{E}\left[\left\|\mathbf{X}_{\mathcal{U}}\right\|_{1}\right] & =\mathrm{E}_{\mathcal{U}},
\end{aligned}
$$

where $\left\{\mathrm{E}_{\mathcal{U}}: \mathcal{U} \in \mathscr{U}\right\}$ satisfies

$$
\sum_{\mathcal{U} \in \mathscr{U}} p_{\mathcal{U}}\left(s_{\mathcal{U}} \mathrm{A}+\mathrm{E}_{\mathcal{U}}\right) \leq \alpha \mathrm{A} .
$$

To further bound the RHS of (193), we use the duality-based upper-bounding technique using a product output distribution

$$
R_{\mathcal{U}}\left(\mathbf{y}_{\mathcal{U}}\right)=\prod_{\ell=1}^{n_{\mathrm{R}}} R_{\mathcal{U}, \ell}\left(y_{\mathcal{U}, \ell}\right) .
$$

Denoting by $W_{\mathcal{U}}\left(\cdot \mid \mathbf{X}_{\mathcal{U}}\right)$ the transition law of the $n_{\mathrm{R}} \times n_{\mathrm{R}}$ MIMO channel with input $\mathbf{X}_{\mathcal{U}}$ and output $\mathbf{Y}_{\mathcal{U}} \triangleq \mathbf{X}_{\mathcal{U}}+\mathbf{Z}_{\mathcal{U}}$, and by $W_{\mathcal{U}, \ell}\left(\cdot \mid X_{\mathcal{U}, \ell}\right)$ the marginal transition law of its $\ell$ th component, we have:

$$
\begin{aligned}
& \mathrm{I}\left(\mathbf{X}_{\mathcal{U}} ; \mathbf{X}_{\mathcal{U}}+\mathbf{Z}_{\mathcal{U}} \mid \mathbb{U}^{\star}=\mathcal{U}\right) \\
& \quad \leq \mathrm{E}_{\mathbf{X}_{\mathcal{U}} \mid \mathbb{U}^{\star}=\mathcal{U}}\left[\mathrm{D}\left(W_{\mathcal{U}}\left(\cdot \mid \mathbf{X}_{\mathcal{U}}\right) \| R_{\mathcal{U}}(\cdot)\right)\right] \\
& \quad=-\mathrm{h}\left(\mathbf{X}_{\mathcal{U}}+\mathbf{Z}_{\mathcal{U}} \mid \mathbf{X}_{\mathcal{U}}, \mathbb{U}^{\star}=\mathcal{U}\right)-\mathrm{E}_{\mathbf{X}_{\mathcal{U}} \mid \mathbb{U}^{\star}=\mathcal{U}}\left[\sum_{\ell=1}^{n_{\mathrm{R}}} \mathrm{E}_{W \mathcal{U}\left(\mathbf{Y}_{\mathcal{U}} \mid \mathbf{X}_{\mathcal{U}}\right)}\left[\log R_{\mathcal{U}, \ell}\left(Y_{\mathcal{U}, \ell}\right)\right]\right. \\
& \quad=-\frac{n_{\mathrm{R}}}{2} \log 2 \pi e+\log \left|\operatorname{det} \mathrm{H}_{\mathcal{U}}\right|-\sum_{\ell=1}^{n_{\mathrm{R}}} \mathrm{E}_{X_{\mathcal{U}, \ell} \mid \mathbb{U}^{\star}=\mathcal{U}}\left[\mathrm{E}_{W_{\mathcal{U}, \ell}\left(Y_{\mathcal{U}, \ell} \mid X_{\mathcal{U}, \ell}\right)}\left[\log R_{\mathcal{U}, \ell}\left(Y_{\mathcal{U}, \ell}\right)\right]\right]
\end{aligned}
$$

where the last equality holds because

$$
\mathrm{h}\left(\mathbf{X}_{\mathcal{U}}+\mathbf{Z}_{\mathcal{U}} \mid \mathbf{X}_{\mathcal{U}}, \mathbb{U}^{\star}=\mathcal{U}\right)=\mathrm{h}\left(\mathbf{Z}_{\mathcal{U}}\right)=\frac{1}{2} \log \left((2 \pi e)^{n_{\mathrm{R}}} \operatorname{det} \mathrm{H}_{\mathcal{U}}^{-1} \mathrm{H}_{\mathcal{U}}^{-\top}\right) .
$$

We finally combine (190) with (193) and (202) to obtain

$$
\begin{aligned}
\mathrm{C}_{\mathrm{H}}(\mathrm{A}, \alpha \mathrm{A}) \leq & \mathrm{H}\left(\mathbf{p}^{*}\right)-\sum_{\ell=1}^{n_{\mathrm{R}}} \sum_{\mathcal{U} \in \mathscr{U}} p_{\mathcal{U}}^{*} \mathrm{E}_{X_{\mathcal{U}, \ell \mid \mathbb{U}^{*}}=\mathcal{U}}\left[\mathrm{E}_{W_{\mathcal{U}, \ell}\left(Y_{\mathcal{U}, \ell} \mid X_{\mathcal{U}, \ell}\right)}\left[\log R_{\mathcal{U}, \ell}\left(Y_{\mathcal{U}, \ell}\right)\right]\right] \\
& +\sum_{\mathcal{U} \in \mathscr{U}} p_{\mathcal{U}}^{*} \log \left|\operatorname{det} \mathrm{H}_{\mathcal{U}}\right|-\frac{n_{\mathrm{R}}}{2} \log 2 \pi e
\end{aligned}
$$

where $\mathbf{p}^{*}$ denotes the probability vector of $\mathbb{U}^{\star}$. The bounds in Section 6.2 are then found by picking appropriate choices for the distribution on the output alphabet $R_{\mathcal{U}, \ell}(\cdot)$. We elaborate on this in the following. 


\section{F.1 Proof of Theorem 17}

Inspired by [11] and [12], we choose

$$
R_{\mathcal{U}, \ell}(y)= \begin{cases}\frac{\beta}{\sqrt{2 \pi} \sigma \mathcal{U}, \ell} \cdot e^{-\frac{y^{2}}{2 \sigma_{\mathcal{U}, \ell}^{2}}} & \text { if } y \in(-\infty, 0), \\ (1-\beta) \cdot \frac{1}{A} & \text { if } y \in[0, \mathrm{~A}], \\ \frac{\beta}{\sqrt{2 \pi} \sigma_{\mathcal{U}, \ell}} \cdot e^{-\frac{(y-A)^{2}}{2 \sigma_{\mathcal{U}, \ell}^{2}}} & \text { if } y \in(\mathrm{A}, \infty),\end{cases}
$$

where $\beta \in(0,1)$ will be specified later. Recall that $\sigma_{\mathcal{U}, \ell}$ is the square root of the $\ell$ th diagonal entry of the matrix $\mathrm{H}_{\mathcal{U}}^{-1} \mathrm{H}_{\mathcal{U}}^{-\top}$, i.e.,

$$
\sigma_{\mathcal{U}, \ell}=\sqrt{\operatorname{Var}\left[Z_{\mathcal{U}, \ell}\right]}
$$

We notice that

$$
\begin{aligned}
- & \int_{-\infty}^{0} W_{\mathcal{U}, \ell}(y \mid x) \log R_{\mathcal{U}, \ell}(y) \mathrm{d} y \\
= & -\int_{-\infty}^{0} \frac{1}{\sqrt{2 \pi} \sigma_{\mathcal{U}, \ell}} e^{-\frac{(y-x)^{2}}{2 \sigma_{\mathcal{U}, \ell}}}\left(\log \frac{\beta}{\sqrt{2 \pi} \sigma_{\mathcal{U}, \ell}}-\frac{y^{2}}{2 \sigma_{\mathcal{U}, \ell}^{2}}\right) \mathrm{d} y \\
= & -\log \left(\frac{\beta}{\sqrt{2 \pi} \sigma_{\mathcal{U}, \ell}}\right) \mathcal{Q}\left(\frac{x}{\sigma_{\mathcal{U}, \ell}}\right)+\frac{1}{2} \mathcal{Q}\left(\frac{x}{\sigma_{\mathcal{U}, \ell}}\right) \\
& +\frac{1}{2}\left(\frac{x}{\sigma_{\mathcal{U}, \ell}}\right)^{2} \mathcal{Q}\left(\frac{x}{\sigma_{\mathcal{U}, \ell}}\right)-\frac{x}{2 \sigma_{\mathcal{U}, \ell}} \phi\left(\frac{x}{\sigma_{\mathcal{U}, \ell}}\right) \\
\leq & -\left(\log \frac{\beta}{\sqrt{2 \pi} \sigma_{\mathcal{U}, \ell}}-\frac{1}{2}\right) \mathcal{Q}\left(\frac{x}{\sigma_{\mathcal{U}, \ell}}\right) \\
= & -\log \frac{\beta}{\sqrt{2 \pi e} \sigma_{\mathcal{U}, \ell}} \cdot \mathcal{Q}\left(\frac{x}{\sigma_{\mathcal{U}, \ell}}\right)
\end{aligned}
$$

where

$$
\phi(x) \triangleq \frac{1}{\sqrt{2 \pi}} e^{-\frac{x^{2}}{2}},
$$

and where (209) holds because of [32, Prop. A.8]

$$
\xi \mathcal{Q}(\xi) \leq \phi(\xi), \quad \xi \geq 0 .
$$

Similarly,

$$
-\int_{\AA}^{\infty} W_{\mathcal{U}, \ell}(y \mid x) \log R_{\mathcal{U}, \ell}(y) \mathrm{d} y \leq-\log \frac{\beta}{\sqrt{2 \pi e} \sigma_{\mathcal{U}, \ell}} \cdot \mathcal{Q}\left(\frac{\mathrm{A}-x}{\sigma_{\mathcal{U}, \ell}}\right) .
$$

Moreover, we have

$$
\begin{aligned}
-\int_{0}^{\mathrm{A}} W_{\mathcal{U}, \ell}(y \mid x) \log R_{\mathcal{U}, \ell}(y) \mathrm{d} y & =-\int_{0}^{\mathrm{A}} \frac{1}{\sqrt{2 \pi} \sigma_{\mathcal{U}, \ell}} e^{-\frac{(y-x)^{2}}{2 \sigma_{\mathcal{U}, \ell}^{2}}} \log \frac{(1-\beta)}{\mathrm{A}} \mathrm{d} y \\
& =\log \left(\frac{\mathrm{A}}{1-\beta}\right) \cdot\left(1-\mathcal{Q}\left(\frac{x}{\sigma_{\mathcal{U}, \ell}}\right)-\mathcal{Q}\left(\frac{\mathrm{A}-x}{\sigma_{\mathcal{U}, \ell}}\right)\right) .
\end{aligned}
$$

We choose

$$
\beta=\frac{\sqrt{2 \pi e} \sigma_{\mathcal{U}, \ell}}{A+\sqrt{2 \pi e} \sigma_{\mathcal{U}, \ell}}
$$


and obtain from (210), (213), and (215)

$$
-\mathrm{E}_{W_{\mathcal{U}, \ell}\left(Y_{\mathcal{U}, \ell} \mid X_{\mathcal{U}, \ell}\right)}\left[\log R_{\mathcal{U}, \ell}\left(Y_{\mathcal{U}, \ell}\right)\right] \leq \log \left(\mathrm{A}+\sqrt{2 \pi e} \sigma_{\mathcal{U}, \ell}\right) .
$$

Substituting (217) into (204) then yields

$$
\begin{aligned}
& \mathrm{C}_{\mathrm{H}}(\mathrm{A}, \alpha \mathrm{A}) \leq \sup _{\mathbf{p}}\left\{\mathrm{H}(\mathbf{p})-\frac{n_{\mathrm{R}}}{2} \log 2 \pi e+\sum_{\mathcal{U} \in \mathscr{U}} p_{\mathcal{U}} \log \left|\operatorname{det} \mathrm{H}_{\mathcal{U}}\right|\right. \\
&\left.+\sum_{\mathcal{U} \in \mathscr{U}} p_{\mathcal{U}} \sum_{\ell=1}^{n_{\mathrm{R}}} \log \left(\mathrm{A}+\sqrt{2 \pi e} \sigma_{\mathcal{U}, \ell}\right)\right\} \\
&=\sup _{\mathbf{p}}\left\{\mathrm{H}(\mathbf{p})+\sum_{\mathcal{U} \in \mathscr{U}} p_{\mathcal{U}} \log \frac{\left|\operatorname{det} \mathrm{H}_{\mathcal{U}}\right|}{\mathrm{V}_{\mathrm{H}}}+\log \mathrm{V}_{\mathrm{H}}\right. \\
&\left.+\sum_{\mathcal{U} \in \mathscr{U}} p_{\mathcal{U}} \sum_{\ell=1}^{n_{\mathrm{R}}} \log \left(\sigma_{\mathcal{U}, \ell}+\frac{\mathrm{A}}{\sqrt{2 \pi e}}\right)\right\} \\
&=\sup _{\mathbf{p}}\left\{\log \mathrm{V}_{\mathrm{H}}-\mathrm{D}(\mathbf{p} \| \mathbf{q})+\sum_{\mathcal{U} \in \mathscr{U}} p_{\mathcal{U}} \sum_{\ell=1}^{n_{\mathrm{R}}} \log \left(\sigma_{\mathcal{U}, \ell}+\frac{\mathrm{A}}{\sqrt{2 \pi e}}\right)\right\} .
\end{aligned}
$$

\section{F.2 Proof of Theorem 18}

We choose

$$
R_{\mathcal{U}, \ell}(y)= \begin{cases}\frac{\beta}{\sqrt{2 \pi} \sigma_{\mathcal{U}, \ell}} e^{-\frac{y^{2}}{2 \sigma_{\mathcal{U}, \ell}}} & \text { if } y \in(-\infty, 0), \\ \frac{1-\beta}{A} \cdot \frac{\mu}{1-e^{-\mu}} e^{-\frac{\mu y}{A}} & \text { if } y \in[0, A], \\ \frac{\beta}{\sqrt{2 \pi} \sigma_{\mathcal{U}, \ell}} e^{-\frac{(y-A)^{2}}{2 \sigma_{\mathcal{U}, \ell}^{2}}} & \text { if } y \in(\mathrm{A}, \infty),\end{cases}
$$

where $\beta \in(0,1)$ and $\mu>0$ will be specified later.

We notice that the inequalities in (210) and (213) still hold, while

$$
\begin{aligned}
-\int_{0}^{\mathrm{A}} & W_{\mathcal{U}, \ell}(y \mid x) \log R_{\mathcal{U}, \ell}(y) \mathrm{d} y \\
= & -\int_{0}^{\mathrm{A}} \frac{1}{\sqrt{2 \pi} \sigma_{\mathcal{U}, \ell}} e^{-\frac{(y-x)^{2}}{2 \sigma_{\mathcal{U}, \ell}^{2}}}\left(\log \frac{1-\beta}{\mathrm{A}} \frac{\mu}{1-e^{-\mu}}-\frac{\mu}{\mathrm{A}} y\right) \mathrm{d} y \\
= & -\log \left(\frac{1-\beta}{\mathrm{A}} \frac{\mu}{1-e^{-\mu}}\right)\left(1-\mathcal{Q}\left(\frac{x}{\sigma_{\mathcal{U}, \ell}}\right)-\mathcal{Q}\left(\frac{\mathrm{A}-x}{\sigma_{\mathcal{U}, \ell}}\right)\right) \\
& +\frac{\mu \sigma_{\mathcal{U}, \ell}}{\mathrm{A}}\left(\phi\left(\frac{x}{\sigma_{\mathcal{U}, \ell}}\right)-\phi\left(\frac{\mathrm{A}-x}{\sigma_{\mathcal{U}, \ell}}\right)\right)+\frac{\mu}{\mathrm{A}} x\left(1-\mathcal{Q}\left(\frac{x}{\sigma_{\mathcal{U}, \ell}}\right)-\mathcal{Q}\left(\frac{\mathrm{A}-x}{\sigma_{\mathcal{U}, \ell}}\right)\right) \\
\leq & -\log \left(\frac{1-\beta}{\mathrm{A}} \frac{\mu}{1-e^{-\mu}}\right)\left(1-\mathcal{Q}\left(\frac{x}{\sigma_{\mathcal{U}, \ell}}\right)-\mathcal{Q}\left(\frac{\mathrm{A}-x}{\sigma_{\mathcal{U}, \ell}}\right)\right) \\
& +\frac{\mu \sigma_{\mathcal{U}, \ell}}{\mathrm{A}}\left(\phi(0)-\phi\left(\frac{\mathrm{A}}{\sigma_{\mathcal{U}, \ell}}\right)\right)+\frac{\mu}{\mathrm{A}} x\left(1-2 \mathcal{Q}\left(\frac{\mathrm{A}}{2 \sigma_{\mathcal{U}, \ell}}\right)\right) \\
\leq & -\log \left(\frac{1-\beta}{\mathrm{A}} \frac{\mu}{1-e^{-\mu}}\right)\left(1-\mathcal{Q}\left(\frac{x}{\sigma_{\mathcal{U}, \ell}}\right)-\mathcal{Q}\left(\frac{\mathrm{A}-x}{\sigma_{\mathcal{U}, \ell}}\right)\right) \\
& +\frac{\mu \sigma_{\mathcal{U}, \ell}}{\mathrm{A}}\left(\phi(0)-\phi\left(\frac{\mathrm{A}}{\sigma_{\mathcal{U}, \ell}}\right)\right)+\frac{\mu}{\mathrm{A}} x .
\end{aligned}
$$

Here (224) follows from the fact that, for $\xi \in[0, A], 1-\mathcal{Q}(\xi)-\mathcal{Q}(A-\xi)$ achieves the maximum value at $\xi=\frac{\AA}{2}$, and that $\phi(\xi)$ is monotonically decreasing; and (225) holds because $1-2 \mathcal{Q}(\xi) \leq 1$ and because $x \geq 0$. 
Combining (210), (213), and (225), and choosing

$$
\beta=\frac{\mu \sqrt{2 \pi e} \sigma_{\mathcal{U}, \ell}}{\mathrm{A}\left(1-e^{-\mu}\right)+\mu \sqrt{2 \pi e} \sigma_{\mathcal{U}, \ell}}
$$

now yield

$$
\begin{aligned}
& -\mathrm{E}_{W_{\mathcal{U}, \ell}\left(Y_{\mathcal{U}, \ell} \mid x_{\mathcal{U}, \ell}\right)}\left[\log R_{\mathcal{U}, \ell}\left(Y_{\mathcal{U}, \ell}\right)\right] \\
& \quad \leq \log \left(\sqrt{2 \pi e} \sigma_{\mathcal{U}, \ell}+\mathrm{A} \cdot \frac{1-e^{-\mu}}{\mu}\right)+\frac{\mu \sigma_{\mathcal{U}, \ell}}{A \sqrt{2 \pi}}\left(1-e^{-\frac{A^{2}}{2 \sigma_{\mathcal{U}, \ell}^{2}}}\right)+\frac{\mu}{A} x_{\mathcal{U}, \ell}
\end{aligned}
$$

Substituting (227) into (204), we have

$$
\begin{aligned}
\mathrm{C}_{\mathrm{H}} & (\mathrm{A}, \alpha \mathrm{A}) \\
\leq & \mathrm{H}\left(\mathbf{p}^{*}\right)+\sum_{\mathcal{U} \in \mathscr{\mathscr { U }}} p_{\mathcal{U}}^{*} \log \left|\operatorname{det} \mathrm{H}_{\mathcal{U}}\right|-\frac{n_{\mathrm{R}}}{2} \log 2 \pi e \\
& +\sum_{\mathcal{U} \in \mathscr{U}} p_{\mathcal{U}}^{*} \sum_{\ell=1}^{n_{\mathrm{R}}} \log \left(\sqrt{2 \pi e} \sigma_{\mathcal{U}, \ell}+\mathrm{A} \cdot \frac{1-e^{-\mu}}{\mu}\right) \\
& +\frac{\mu}{\mathrm{A} \sqrt{2 \pi}} \sum_{\mathcal{U} \in \mathscr{U}} p_{\mathcal{U}}^{*} \sum_{\ell=1}^{n_{\mathrm{R}}} \sigma_{\mathcal{U}, \ell}\left(1-e^{-\frac{\mathrm{A}^{2}}{2 \sigma_{\mathcal{U}, \ell}^{2}}}\right) \\
& +\frac{\mu}{\mathrm{A}} \sum_{\mathcal{U} \in \mathscr{U}} p_{\mathcal{U}}^{*} \sum_{\ell=1}^{n_{\mathrm{R}}} \mathrm{E}\left[X_{\mathcal{U}, \ell} \mid \mathbb{U}^{*}=\mathcal{U}\right] \\
= & \mathrm{H}\left(\mathbf{p}^{*}\right)+\sum_{\mathcal{U} \in \mathscr{U}} p_{\mathcal{U}}^{*} \log \frac{\left|\operatorname{det} \mathrm{H}_{\mathcal{U}}\right|}{\mathrm{V}_{\mathrm{H}}}+\log \mathbf{V}_{\mathrm{H}}+\sum_{\mathcal{U} \in \mathscr{U}} p_{\mathcal{U}}^{*} \sum_{\ell=1}^{n_{\mathrm{R}}} \log \left(\sigma_{\mathcal{U}, \ell}+\frac{\mathrm{A}}{\sqrt{2 \pi e}} \cdot \frac{1-e^{-\mu}}{\mu}\right) \\
& +\frac{\mu}{\mathrm{A} \sqrt{2 \pi}} \sum_{\mathcal{U} \in \mathscr{U}} p_{\mathcal{U}}^{*} \sum_{\ell=1}^{n_{\mathrm{R}}} \sigma_{\mathcal{U}, \ell}\left(1-e^{-\frac{\mathrm{A}^{2}}{2 \sigma_{\mathcal{U}, \ell}^{2}}}\right)+\frac{\mu}{\mathrm{A}} \sum_{\mathcal{U} \in \mathscr{U}} p_{\mathcal{U}}^{*}\left\|\mathrm{H}_{\mathcal{U}}^{-1}\left(\mathrm{E}\left[\mathbf{X}^{\star} \mid \mathbb{U}^{\star}=\mathcal{U}\right]-\mathbf{v}_{\mathcal{U}}\right)\right\|_{1} \\
\leq & \log \mathbf{V}_{\mathrm{H}}-\mathrm{D}\left(\mathbf{p}^{*} \| \mathbf{q}\right)+\sum_{\mathcal{U} \in \mathscr{U}} p_{\mathcal{U}}^{*} \sum_{\ell=1}^{n_{\mathrm{R}}} \log \left(\sigma_{\mathcal{U}, \ell}+\frac{\mathrm{A}}{\sqrt{2 \pi e}} \cdot \frac{1-e^{-\mu}}{\mu}\right) \\
& +\frac{\mu}{\mathrm{A} \sqrt{2 \pi}} \sum_{\mathcal{U} \in \mathscr{U}} p_{\mathcal{U}}^{*} \sum_{\ell=1}^{n_{\mathrm{R}}} \sigma_{\mathcal{U}, \ell}\left(1-e^{-\frac{\mathrm{A}^{2}}{2 \sigma_{\mathcal{U}}^{2}, \ell}}\right)+\mu\left(\alpha-\sum_{\mathcal{U} \in \mathscr{U}} p_{\mathcal{U}}^{*} s_{\mathcal{U}}\right),
\end{aligned}
$$

where (229) follows from (195), and (230) from (48). Theorem 18 is proven by taking the supremum over the probability vector $\mathbf{p}$ and the infimum over $\mu>0$.

\section{F.3 Proof of Theorem 19}

We choose

$$
R_{\mathcal{U}, \ell}(y)= \begin{cases}\frac{1}{\sqrt{2 \pi} \sigma_{\mathcal{U}, \ell}} e^{-\frac{y^{2}}{2 \sigma_{\mathcal{U}}, \ell}} & \text { if } y \in(-\infty,-\delta), \\ \frac{\mu}{A} \cdot \frac{1-2 \mathcal{Q}\left(\frac{\delta}{\sigma \mathcal{U}, \ell}\right)}{e^{\frac{\mu \delta}{A}}-e^{-\mu\left(1+\frac{\delta}{A}\right)}} e^{-\frac{\mu y}{A}} & \text { if } y \in[-\delta, A+\delta], \\ \frac{1}{\sqrt{2 \pi} \sigma_{\mathcal{U}, \ell}} e^{-\frac{(y-A)^{2}}{2 \sigma_{\mathcal{U}}, \ell}} & \text { if } y \in(A+\delta, \infty),\end{cases}
$$

where $\delta, \mu>0$ are free parameters. Following the steps in the proof of [10, App. B.B] and bounding $1-\mathcal{Q}\left(\xi_{1}\right)-\mathcal{Q}\left(\xi_{2}\right) \leq 1$, we obtain:

$$
\begin{aligned}
& -\mathrm{E}_{X_{\mathcal{U}, \ell \mid \mathbb{U}^{*}}=\mathcal{U}}\left[\mathrm{E}_{W_{\mathcal{U}, \ell}\left(Y_{\mathcal{U}, \ell} \mid X_{\mathcal{U}, \ell}\right.}\left[\log R_{\mathcal{U}, \ell}\left(Y_{\mathcal{U}, \ell}\right)\right]\right] \\
& \quad \leq \log \left(A \cdot \frac{e^{\frac{\mu \delta}{A}}-e^{-\mu\left(1+\frac{\delta}{A}\right)}}{\mu\left(1-2 \mathcal{Q}\left(\frac{\delta}{\sigma_{\mathcal{U}, \ell}}\right)\right)}\right)+\frac{\delta}{\sqrt{2 \pi} \sigma_{\mathcal{U}, \ell}} e^{-\frac{\delta^{2}}{2 \sigma_{\mathcal{U}, \ell}^{2}}}+\mathcal{Q}\left(\frac{\delta}{\sigma_{\mathcal{U}, \ell}}\right)
\end{aligned}
$$




$$
+\frac{\mu \sigma_{\mathcal{U}, \ell}}{A \sqrt{2 \pi}}\left(e^{-\frac{\delta^{2}}{2 \sigma_{\mathcal{U}, \ell}^{2}}}-e^{-\frac{(\mathrm{A}+\delta)^{2}}{2 \sigma_{\mathcal{U}}^{2}, \ell}}\right)+\frac{\mu}{\mathrm{A}} \mathrm{E}\left[X_{\mathcal{U}, \ell} \mid \mathbb{U}^{\star}=\mathcal{U}\right] .
$$

Plugging (232) into (204) and using a derivation analogous to (228)-(230) then results in the given bound.

\section{F.4 Proof of Theorem 20}

Using that

$$
\mathbf{h}(\mathbf{Y}) \leq \frac{1}{2} \log \left((2 \pi e)^{n_{\mathrm{R}}} \operatorname{det} \mathrm{K}_{\mathbf{Y Y}}\right),
$$

where

$$
\mathrm{K}_{\mathbf{Y Y}}=\mathrm{K}_{\overline{\mathbf{X}} \overline{\mathrm{X}}}+\mathrm{I}
$$

we have

$$
\begin{aligned}
& \mathrm{C}_{\mathrm{H}}(\mathrm{A}, \alpha \mathrm{A})=\max _{P_{\mathbf{X}}}\{\mathrm{h}(\mathbf{Y})-\mathrm{h}(\mathbf{Z})\} \\
& \leq \max _{P \mathbf{X}}\left\{\frac{1}{2} \log \left((2 \pi e)^{n_{\mathrm{R}}} \operatorname{det}\left(\mathrm{K}_{\overline{\mathbf{X}} \overline{\mathbf{x}}}+\mathrm{I}\right)\right)-\frac{1}{2} \log (2 \pi e)^{n_{\mathrm{R}}}\right\} \\
& =\max _{P \mathbf{x}} \frac{1}{2} \log \operatorname{det}\left(I+\mathrm{K}_{\overline{\mathbf{x}} \overline{\mathbf{x}}}\right) \\
& \leq \max _{P_{\mathbf{X}}} \frac{1}{2} \log \prod_{i=1}^{n_{\mathrm{R}}}\left(\mathrm{I}+\mathrm{K}_{\overline{\mathbf{X}} \overline{\mathbf{x}}}\right)_{i, i} \\
& =\max _{P_{\mathbf{X}}} \frac{n_{\mathrm{R}}}{2} \sum_{i=1}^{n_{\mathrm{R}}} \frac{1}{n_{\mathrm{R}}} \log \left(1+\left(\mathrm{K}_{\overline{\mathbf{X}} \overline{\mathbf{X}}}\right)_{i, i}\right) \\
& \leq \max _{P \mathbf{x}} \frac{n_{\mathrm{R}}}{2} \log \left(1+\sum_{i=1}^{n_{\mathrm{R}}} \frac{1}{n_{\mathrm{R}}}\left(\mathrm{K}_{\overline{\mathbf{x}} \overline{\mathbf{x}}}\right)_{i, i}\right) \\
& =\max _{P_{\mathbf{X}}} \frac{n_{\mathrm{R}}}{2} \log \left(1+\frac{1}{n_{\mathrm{R}}} \operatorname{tr}\left(\mathrm{K}_{\overline{\mathbf{X}} \overline{\mathbf{X}}}\right)\right) \\
& =\frac{n_{\mathrm{R}}}{2} \log \left(1+\frac{1}{n_{\mathrm{R}}} \max _{P_{\mathbf{X}}} \operatorname{tr}\left(\mathrm{K}_{\overline{\mathbf{x}} \overline{\mathbf{x}}}\right)\right) \text {. }
\end{aligned}
$$

Here, (238) follows from Hadamard's inequality, and (240) follows from Jensen's inequality.

\section{G Derivation of Asymptotic Results}

\section{G.1 Proof of Theorem 21}

It follows directly from Theorem 15 that the RHS of (85) is a lower bound to its LHS. To prove the other direction, using that $\mathrm{D}(\mathbf{p} \| \mathbf{q}) \geq 0$, we have from Theorem 17 that

$$
\mathrm{C}_{\mathrm{H}}(\mathrm{A}, \alpha \mathrm{A}) \leq \log \mathrm{V}_{\mathrm{H}}+n_{\mathrm{R}} \log \left(\sigma_{\max }+\frac{\mathrm{A}}{\sqrt{2 \pi e}}\right)
$$

where

$$
\sigma_{\max } \triangleq \max _{\substack{\mathcal{U} \in \mathscr{U} \\ \ell \in\left\{1, \ldots, n_{\mathrm{R}}\right\}}} \sigma_{\mathcal{U}, \ell}
$$

This proves that the RHS of (85) is also an upper bound to its LHS, and hence completes the proof of $(85)$. 
Next, we prove (86). Again, that its RHS is a lower bound to its LHS follows immediately from Theorem 16 . To prove the other direction, we define for any $\mathbf{p}$ :

$$
\lambda(\mathbf{p}) \triangleq \alpha-\sum_{\mathcal{U} \in \mathscr{U}} p_{\mathcal{U}} s_{\mathcal{U}} \leq \alpha .
$$

We then fix $A \geq 1$ and choose $\mu$ depending on $\lambda(\mathbf{p})$ to be

$$
\mu= \begin{cases}\mu^{*}(\mathbf{p}) & \text { if } A^{-(1-\zeta)}<\frac{\lambda(\mathbf{p})}{n_{\mathrm{R}}}<\frac{1}{2}, \\ A^{1-\zeta} & \text { if } \frac{\lambda(\mathbf{p})}{n_{\mathrm{R}}} \leq A^{-(1-\zeta)}, \\ \frac{1}{A} & \text { if } \frac{\lambda(\mathbf{p})}{n_{\mathrm{R}}} \geq \frac{1}{2},\end{cases}
$$

where $0<\zeta<1$ is a free parameter and $\mu^{*}(\mathbf{p})$ is the unique solution to

$$
\frac{1}{\mu^{*}}-\frac{e^{-\mu^{*}}}{1-e^{-\mu^{*}}}=\frac{\lambda(\mathbf{p})}{n_{\mathrm{R}}} .
$$

Note that in the first case of (246),

$$
\mathrm{A}^{-(1-\zeta)}<\frac{\lambda(\mathbf{p})}{n_{\mathrm{R}}}=\frac{1}{\mu^{*}(\mathbf{p})}-\frac{e^{-\mu^{*}(\mathbf{p})}}{1-e^{-\mu^{*}(\mathbf{p})}}<\frac{1}{\mu^{*}(\mathbf{p})},
$$

i.e.,

$$
\mu^{*}(\mathbf{p})<A^{1-\zeta}
$$

and thus the choice (246) makes sure that in all three cases, irrespective of $\mathbf{p}$ :

$$
\mu \leq A^{1-\zeta}, \quad \text { for } A \geq 1
$$

Then, for $A \geq 1$, the upper bound (81) can be loosened as follows:

$$
\mathrm{C}_{\mathrm{H}}(\mathrm{A}, \alpha \mathrm{A}) \leq \frac{1}{2} \log \left(\frac{\mathrm{A}^{2 n_{\mathrm{R}}} \mathrm{V}_{\mathrm{H}}^{2}}{(2 \pi e)^{n_{\mathrm{R}}}}\right)+f(\mathrm{~A})+\sup _{\mathbf{p}} g(\mathrm{~A}, \mathbf{p}, \mu)
$$

where

$$
\begin{aligned}
& f(\mathrm{~A}) \triangleq \frac{n_{\mathrm{R}} \sigma_{\max }}{\mathrm{A}^{\zeta} \sqrt{2 \pi}}\left(1-e^{-\frac{\mathrm{A}^{2}}{2 \sigma_{\min }^{2}}}\right), \\
& g(\mathrm{~A}, \mathbf{p}, \mu) \triangleq n_{\mathrm{R}} \log \left(\frac{\sqrt{2 \pi e} \sigma_{\max }}{\mathrm{A}}+\frac{1-e^{-\mu}}{\mu}\right)+\mu \lambda(\mathbf{p})-\mathrm{D}(\mathbf{p} \| \mathbf{q})
\end{aligned}
$$

with $\sigma_{\max }$ defined in $(244)$ and with

$$
\sigma_{\min } \triangleq \min _{\substack{\mathcal{U} \in \mathscr{U} \\ \ell \in\left\{1, \ldots, n_{\mathrm{R}}\right\}}} \sigma_{\mathcal{U}, \ell}
$$

Note that

$$
\lim _{A \rightarrow \infty} f(A)=0
$$

Next, we upper-bound $g(A, \mathbf{p}, \mu)$ individually for each of the three different cases in (246) to obtain a bound of the form

$$
g(\mathrm{~A}, \mathbf{p}, \mu) \leq \begin{cases}g_{1}(\mathrm{~A}) & \text { if } \mathrm{A}^{-(1-\zeta)}<\frac{\lambda(\mathbf{p})}{n_{\mathrm{R}}}<\frac{1}{2}, \\ g_{2}(\mathrm{~A}) & \text { if } \frac{\lambda(\mathbf{p})}{n_{\mathrm{R}}} \leq \mathrm{A}^{-(1-\zeta)} \\ g_{3}(\mathrm{~A}) & \text { if } \frac{\lambda(\mathbf{p})}{n_{\mathrm{R}}} \geq \frac{1}{2}\end{cases}
$$


for three functions $g_{1}, g_{2}$, and $g_{3}$ that only depend on $A$ but not on $\mathbf{p}$ or $\mu$. Thus, we shall then obtain the bound

$$
g(\mathrm{~A}, \mathbf{p}, \mu) \leq \max \left\{g_{1}(\mathrm{~A}), g_{2}(\mathrm{~A}), g_{3}(\mathrm{~A})\right\}, \quad \mathrm{A} \geq 1 .
$$

The functions $g_{1}, g_{2}$, and $g_{3}$ are introduced in the following.

For the first case where $\frac{\lambda(\mathbf{p})}{n_{\mathrm{R}}} \in\left(A^{-(1-\zeta)}, \frac{1}{2}\right)$, we have

$$
\begin{aligned}
& g(\mathrm{~A}, \mathbf{p}, \mu)=n_{\mathrm{R}} \log \left(\frac{\sqrt{2 \pi e} \sigma_{\max }}{\mathrm{A}}+\frac{1-e^{-\mu^{*}(\mathbf{p})}}{\mu^{*}(\mathbf{p})}\right)+\mu^{*}(\mathbf{p}) \lambda(\mathbf{p})-\mathrm{D}(\mathbf{p} \| \mathbf{q}) \\
& =n_{\mathrm{R}} \log \left(1+\frac{\mu^{*}(\mathbf{p})}{1-e^{-\mu^{*}(\mathbf{p})}} \cdot \frac{\sqrt{2 \pi e} \sigma_{\max }}{\mathrm{A}}\right) \\
& +n_{\mathrm{R}}\left(1-\log \left(\frac{\mu^{*}(\mathbf{p})}{1-e^{-\mu^{*}(\mathbf{p})}}\right)-\frac{\mu^{*}(\mathbf{p}) e^{-\mu^{*}(\mathbf{p})}}{1-e^{-\mu^{*}(\mathbf{p})}}\right)-\mathrm{D}(\mathbf{p} \| \mathbf{q}) \\
& \leq \sup _{\mathbf{p}: \frac{\lambda(\mathbf{p})}{n_{\mathrm{R}}} \in\left(A^{\zeta-1}, \frac{1}{2}\right)}\left\{-\mathrm{D}(\mathbf{p} \| \mathbf{q})+n_{\mathrm{R}} \log \left(1+\frac{\mu^{*}(\mathbf{p})}{1-e^{-\mu^{*}(\mathbf{p})}} \cdot \frac{\sqrt{2 \pi e} \sigma_{\max }}{\mathrm{A}}\right)\right. \\
& \left.+n_{\mathrm{R}}\left(1-\log \left(\frac{\mu^{*}(\mathbf{p})}{1-e^{-\mu^{*}(\mathbf{p})}}\right)-\frac{\mu^{*}(\mathbf{p}) e^{-\mu^{*}(\mathbf{p})}}{1-e^{-\mu^{*}(\mathbf{p})}}\right)\right\}
\end{aligned}
$$

$\triangleq g_{1}(A)$.

Here, in (259) we have used (247).

For the second case where $\frac{\lambda(\mathbf{p})}{n_{\mathrm{R}}} \leq A^{-(1-\zeta)}$, we use this inequality in combination with (246) to bound

$$
\mu \lambda(\mathbf{p}) \leq A^{1-\zeta} \cdot n_{\mathrm{R}} A^{-(1-\zeta)}=n_{\mathrm{R}}
$$

Because $\mathrm{D}(\mathbf{p} \| \mathbf{q}) \geq 0$, we thus obtain

$$
\begin{aligned}
g(\mathrm{~A}, \mathbf{p}, \mu) & \leq n_{\mathrm{R}} \log \left(\frac{\sqrt{2 \pi e} \sigma_{\max }}{A}+\frac{1-e^{-\mu}}{\mu}\right)+n_{\mathrm{R}} \\
& =n_{\mathrm{R}} \log \left(\frac{\sqrt{2 \pi e} \sigma_{\max }}{A}+\frac{1-e^{-\mathrm{A}^{1-\zeta}}}{\mathrm{A}^{1-\zeta}}\right)+n_{\mathrm{R}} \\
& \triangleq g_{2}(\mathrm{~A}) .
\end{aligned}
$$

For the third case where $\frac{\lambda(\mathbf{p})}{n_{\mathrm{R}}} \geq \frac{1}{2}$, we have

$$
\begin{aligned}
g(\mathrm{~A}, \mathbf{p}, \mu) & =n_{\mathrm{R}} \log \left(\frac{\sqrt{2 \pi e} \sigma_{\max }}{\mathrm{A}}+\frac{1-e^{-\frac{1}{A}}}{\frac{1}{\mathrm{~A}}}\right)+\frac{\lambda(\mathbf{p})}{\mathrm{A}}-\mathrm{D}(\mathbf{p} \| \mathbf{q}) \\
& \leq n_{\mathrm{R}} \log \left(\frac{\sqrt{2 \pi e} \sigma_{\max }}{\mathrm{A}}+\frac{1-e^{-\frac{1}{A}}}{\frac{1}{\mathrm{~A}}}\right)+\frac{\alpha}{\mathrm{A}}-\inf _{\mathbf{p}: \frac{\lambda(\mathbf{p})}{n_{\mathrm{R}}}>\frac{1}{2}} \mathrm{D}(\mathbf{p} \| \mathbf{q}) \\
& \triangleq g_{3}(\mathrm{~A}) .
\end{aligned}
$$

Here, we used (245) to bound $\lambda(\mathbf{p}) \leq \alpha$.

We have now established (257) for the three functions defined in (261), (265), and (268), respectively. We now analyze the maximum in (257) when $A \rightarrow \infty$. Since $g_{2}(A)$ tends to $-\infty$ as $A \rightarrow \infty$, and since $g_{1}(A)$ and $g_{3}(A)$ are both bounded from below for $A \geq 1$, we know that, for large enough $A, g_{2}(A)$ is strictly smaller than $\max \left\{g_{1}(\mathrm{~A}), g_{3}(\mathrm{~A})\right\}$.

We next look at $g_{3}(A)$ when $A \rightarrow \infty$. Note that

$$
\lim _{A \rightarrow \infty} \frac{1-e^{-\frac{1}{A}}}{\frac{1}{A}}=1,
$$


therefore

$$
\begin{aligned}
\lim _{A \rightarrow \infty} g_{3}(\mathrm{~A}) & =-\inf _{\mathbf{p}: \frac{\lambda(\mathbf{p})}{n_{\mathrm{R}}}>\frac{1}{2}} \mathrm{D}(\mathbf{p} \| \mathbf{q}) \\
& =-\inf _{\mathbf{p}: \alpha-\sum_{\mathcal{U} \in \mathscr{U}} p_{\mathcal{U}} s_{\mathcal{U}} \geq \frac{n_{\mathrm{R}}}{2}} \mathrm{D}(\mathbf{p} \| \mathbf{q}) \\
& =-\operatorname{pinf}_{\mathbf{p}: \alpha-\sum_{\mathcal{U} \in \mathscr{U}} p_{\mathcal{U}} s_{\mathcal{U}}=\frac{n_{\mathrm{R}}}{2}} \mathrm{D}(\mathbf{p} \| \mathbf{q}),
\end{aligned}
$$

where the last equality holds because given $\alpha<\alpha_{\mathrm{th}}$, an optimal $\mathbf{p}$ will meet the constraint with equality.

It remains to investigate the behavior of $g_{1}(\mathrm{~A})$ when $\mathrm{A} \rightarrow \infty$. To this end, we define

$$
\begin{aligned}
\tilde{g}_{1}(\mathrm{~A}, \mathbf{p}) \triangleq & -\mathrm{D}(\mathbf{p} \| \mathbf{q})+n_{\mathrm{R}} \log \left(1+\frac{\mu^{*}(\mathbf{p})}{1-e^{-\mu^{*}(\mathbf{p})}} \cdot \frac{\sqrt{2 \pi e} \sigma_{\max }}{\mathrm{A}}\right) \\
& +n_{\mathrm{R}}\left(1-\log \left(\frac{\mu^{*}(\mathbf{p})}{1-e^{-\mu^{*}(\mathbf{p})}}\right)-\frac{\mu^{*}(\mathbf{p}) e^{-\mu^{*}(\mathbf{p})}}{1-e^{-\mu^{*}(\mathbf{p})}}\right),
\end{aligned}
$$

and note that, for any fixed $\mathbf{p}$,

$$
\Delta(A, \mathbf{p}) \triangleq \tilde{g}_{1}(A, \mathbf{p})-\lim _{A \rightarrow \infty} \tilde{g}_{1}(A, \mathbf{p})=\log \left(1+\frac{\mu^{*}(\mathbf{p})}{1-e^{-\mu^{*}(\mathbf{p})}} \cdot \frac{\sqrt{2 \pi e} \sigma_{\max }}{A}\right) .
$$

Since, when $A \rightarrow \infty$,

$$
|\Delta(A, \mathbf{p})| \leq \log \left(1+\left|\frac{1}{1-e^{-A^{1-\zeta}}} \cdot \frac{\sqrt{2 \pi e} \sigma_{\max }}{A^{\zeta}}\right|\right) \rightarrow \log (1)=0,
$$

we see that $\tilde{g}_{1}(\mathrm{~A}, \mathbf{p})$ converges uniformly over $\mathbf{p}$ as $\mathrm{A} \rightarrow \infty$, and therefore we are allowed to interchange limit and supremum:

$$
\begin{aligned}
& \lim _{A \rightarrow \infty} g_{1}(A) \\
& =\lim _{\boldsymbol{A} \rightarrow \infty} \sup _{\mathbf{p}: \frac{\lambda(\mathbf{p})}{n_{\mathrm{R}}} \in\left(\mathrm{A}^{\zeta-1}, \frac{1}{2}\right)} \tilde{g}_{1}(\boldsymbol{A}, \mathbf{p}) \\
& =\sup _{\mathbf{p}: \frac{\lambda(\mathbf{p})}{n_{\mathrm{R}}} \in\left(0, \frac{1}{2}\right)} \lim _{A \rightarrow \infty} \tilde{g}_{1}(\boldsymbol{A}, \mathbf{p}) \\
& =\sup _{\mathbf{p}: \frac{\lambda(\mathbf{p})}{n_{\mathrm{R}}} \in\left(0, \frac{1}{2}\right)}\left\{n_{\mathrm{R}}\left(1-\log \frac{\mu^{*}(\mathbf{p})}{1-e^{-\mu^{*}(\mathbf{p})}}-\frac{\mu^{*}(\mathbf{p}) e^{-\mu^{*}(\mathbf{p})}}{1-e^{-\mu^{*}(\mathbf{p})}}\right)-\mathrm{D}(\mathbf{p} \| \mathbf{q})\right\} \\
& =\sup _{\mathbf{p}: \lambda(\mathbf{p}) \in\left(\max \left\{0, \frac{n_{\mathrm{R}}}{2}+\alpha-\alpha_{\mathrm{th}}\right\}, \min \left\{\frac{n_{\mathrm{R}}}{2}, \alpha\right\}\right)}\left\{n_{\mathrm{R}}\left(1-\log \frac{\mu^{*}(\mathbf{p})}{1-e^{-\mu^{*}(\mathbf{p})}}-\frac{\mu^{*}(\mathbf{p}) e^{-\mu^{*}(\mathbf{p})}}{1-e^{-\mu^{*}(\mathbf{p})}}\right)\right. \\
& -\mathrm{D}(\mathbf{p} \| \mathbf{q})\} \\
& =\nu \text {. }
\end{aligned}
$$

Here, in (279) we are allowed to restrict the supremum ${ }^{1}$ to $\lambda(\mathbf{p}) \in\left(\frac{n_{\mathrm{R}}}{2}+\alpha-\alpha_{\mathrm{th}}, \alpha\right)$ because of (245) and because

$$
\lambda(\mathbf{q}) \triangleq \alpha-\sum_{\mathcal{U} \in \mathscr{U}} s_{\mathcal{U}} q_{\mathcal{U}}=\alpha-\alpha_{\mathrm{th}}+\frac{n_{\mathrm{R}}}{2}
$$

\footnotetext{
${ }^{1}$ Notice that because of the supremum and continuity, we can restrict to the open interval instead of the closed interval.
} 
and for any $\mathbf{p}$ such that $\lambda(\mathbf{p}) \leq \lambda(\mathbf{q})$ the objective function in (278) is smaller than for $\mathbf{p}=\mathbf{q}$. In fact, $-\mathrm{D}(\mathbf{p} \| \mathbf{q})$ is clearly maximized for $\mathbf{p}=\mathbf{q}$ and

$$
\mu^{*}(\mathbf{p}) \mapsto n_{\mathrm{R}}\left(1-\log \frac{\mu^{*}(\mathbf{p})}{1-e^{-\mu^{*}(\mathbf{p})}}-\frac{\mu^{*}(\mathbf{p}) e^{-\mu^{*}(\mathbf{p})}}{1-e^{-\mu^{*}(\mathbf{p})}}\right)
$$

is decreasing in $\mu^{*}(\mathbf{p})$, which is a decreasing function of $\lambda(\mathbf{p})$; see (247). Finally, (280) follows from the definition of $\nu$ in $(77)$.

It is straightforward to see that $\nu$ is larger than the RHS of (272). Therefore,

$$
\lim _{\mathrm{A} \rightarrow \infty} \max \left\{g_{1}(\mathrm{~A}), g_{2}(\mathrm{~A}), g_{3}(\mathrm{~A})\right\}=\nu .
$$

Combining (251) with (255), (257), and (283) proves the theorem.

\section{G.2 Proof of Theorem 22}

From [28, Corollary 2], it is known that the capacity is lower-bounded as

$$
\mathrm{C}_{\mathrm{H}}(\mathrm{A}, \alpha \mathrm{A}) \geq \frac{1}{2} \max _{P_{\overline{\mathbf{X}}}} \operatorname{tr}\left(\mathrm{K}_{\overline{\mathbf{X}} \overline{\mathbf{X}}}\right)+o\left(\max _{P_{\overline{\mathbf{X}}}} \operatorname{tr}\left(\mathrm{K}_{\overline{\mathbf{X}} \overline{\mathbf{X}}}\right)\right) .
$$

For an upper bound, we use that

$$
\log (1+\xi) \leq \xi, \quad \xi>0,
$$

and obtain from Theorem 20 that

$$
\mathrm{C}_{\mathrm{H}}(\mathrm{A}, \alpha \mathrm{A}) \leq \frac{1}{2} \max _{P_{\overline{\mathbf{x}}}} \operatorname{tr}\left(\mathrm{K}_{\overline{\mathbf{X}} \overline{\mathbf{x}}}\right) .
$$

The theorem is proven by normalizing $\overline{\mathbf{X}}$ by $A$, which results in a factor $A^{2}$, and by then letting $A$ go to zero.

\section{References}

[1] Robert M. Gagliardi and Sherman Karp, Optical Communications. New York, NY, USA: Wiley-Interscience, 1976.

[2] Charles Cox, Edward Ackerman, Roger Helkey, and Gary E. Betts, "Directdetection analog optical links," IEEE Trans. Microwave Theory 8 Tech., vol. 45, no. 8, pp. 1375-1383, Aug. 1997.

[3] Sian C. Jeffrey Lee, Sebastian Randel, Florian Breyer, and Antonius M.J. Koonen, "PAM-DMT for intensity-modulated and direct-detection optical communication systems," IEEE Photonics Tech. Lett., vol. 21, no. 23, pp. 1749-1751, Sept. 2009.

[4] John R. Barry, Wireless Infrared Communications. New York, NY, USA: Springer Science \& Business, 1994.

[5] Mohammad Ali Khalighi and Murat Uysal, "Survey on free space optical communication: A communication theory perspective," IEEE Comm. Surveys \& Tutorials, vol. 16, no. 4, pp. 2231-2258, fourth quarter 2014.

[6] Shane M. Haas and Jeffrey H. Shapiro, "Capacity of wireless optical communications," IEEE J. Select. Areas Commun., vol. 21, no. 8, pp. 1346-1357, Oct. 2003.

[7] Dilukshan Karunatilaka, Fahad Zafar, Vineetha Kalavally, and Rajendran Parthiban, "LED based indoor visible light communications: State of the art," IEEE Comm. Surveys \& Tutorials, vol. 17, no. 3, pp. 1649-1678, third quarter 2015 . 
[8] Murat Uysal and Hatef Nouri, "Optical wireless communications - an emerging technology," in Proc. IEEE Int. Conf. Transp. Optical Netw., Graz, Austria, Jul. 6-10, 2014, pp. 1-7.

[9] Michèle A. Wigger, "Bounds on the capacity of free-space optical intensity channels," Master's thesis, Signal and Inf. Proc. Lab., ETH Zürich, Switzerland, Mar. 2003.

[10] Amos Lapidoth, Stefan M. Moser, and Michèle A. Wigger, "On the capacity of free-space optical intensity channels," IEEE Trans. Inf. Theory, vol. 55, no. 10, pp. 4449-4461, Oct. 2009.

[11] Andrew L. McKellips, "Simple tight bounds on capacity for the peak-limited discrete-time channel," in Proc. IEEE Int. Symp. Inf. Theory, Chicago, IL, USA, Jun. 27 - Jul. 2, 2004, p. 348.

[12] Andrew Thangaraj, Gerhard Kramer, and Georg Böcherer, "Capacity bounds for discrete-time, amplitude-constrained, additive white Gaussian noise channels," IEEE Trans. Inf. Theory, vol. 63, no. 7, pp. 4172-4182, Jul. 2017.

[13] Borzoo Rassouli and Bruno Clerckx, "An upper bound for the capacity of amplitudeconstrained scalar AWGN channel," IEEE Commun. Lett., vol. 20, no. 10, pp. 1924-1926, Oct. 2016.

[14] Stefan M. Moser, Ligong Wang, and Michèle Wigger, "Asymptotic high-SNR capacity of MISO optical intensity channels," in Proc. IEEE Inf. Theory Workshop, Kaohsiung, Taiwan, Nov. 6-10, 2017, pp. 86-90.

[15] Stefan M. Moser, Ligong Wang, and Michèle Wigger, "Capacity results on multipleinput single-output wireless optical channels," IEEE Trans. Inf. Theory, vol. 64, no. 11, pp. 6954-6966, Nov. 2018.

[16] Stefan M. Moser, Michail Mylonakis, Ligong Wang, and Michèle Wigger, "Asymptotic capacity results for MIMO wireless optical communication," in Proc. IEEE Int. Symp. Inf. Theory, Aachen, Germany, Jun. 25-30, 2017, pp. 536-540.

[17] Anas Chaaban, Zouheir Rezki, and Mohamed-Slim Alouini, "MIMO intensitymodulation channels: Capacity bounds and high SNR characterization," in Proc. IEEE Int. Conf. Commun., Paris, France, May 21-25, 2017, pp. 1-6.

[18] Anas Chaaban, Zouheir Rezki, and Mohamed-Slim Alouini, "Capacity bounds and high-SNR capacity of MIMO intensity-modulation optical channels," IEEE Trans. Wireless Commun., vol. 17, no. 5, pp. 3003-3017, May 2018.

[19] Alex Dytso, Mario Goldenbaum, Shlomo Shamai, and H. Vincent Poor, "Upper and lower bounds on the capacity of amplitude-constrained MIMO channels," in Proc. IEEE Global Commun. Conf., Singapore, Dec. 4-8, 2017, pp. 1-6.

[20] Anas Chaaban, Zouheir Rezki, and Mohamed-Slim Alouini, "Low-SNR asymptotic capacity of MIMO optical intensity channels with peak and average constraints," IEEE Trans. Commun., vol. 66, no. 10, pp. 4694-4705, Oct. 2018.

[21] Zixiong Wang, Wen-De Zhong, Songnian Fu, and Chinlon Lin, "Performance comparison of different modulation formats over free-space optical (FSO) turbulence links with space diversity reception technique," IEEE Photonics J., vol. 1, no. 6, pp. 277-285, Dec. 2009.

[22] Ertugrul Başar, Erdal Panayirci, Murat Uysal, and Harald Haas, "Generalized LED index modulation optical OFDM for MIMO visible light communications systems," in Proc. IEEE Int. Conf. Commun., Kuala Lumpur, Malaysia, May 23-27, 2016, pp. 1-5. 
[23] Anil Yesilkaya, Ertugrul Basar, Farshad Miramirkhani, Erdal Panayirci, Murat Uysal, and Harald Haas, "Optical MIMO-OFDM with generalized LED index modulation," IEEE Trans. Commun., vol. 65, no. 8, pp. 3429-3441, Aug. 2017.

[24] Shane M. Haas, Jeffrey H. Shapiro, and Vahid Tarokh, "Space-time codes for wireless optical communications," EURASIP J. App. Sig. Proc., no. 3, pp. 211-220, 2002.

[25] Ehsan Bayaki and Robert Schober, "On space-time coding for free-space optical systems," IEEE Trans. Commun., vol. 58, no. 1, pp. 58-62, Jan. 2010.

[26] Xuegui Song and Julian Cheng, "Subcarrier intensity modulated MIMO optical communications in atmospheric turbulence," IEEE/OSA J. of Optical Comm. \& Networking, vol. 5, no. 9, pp. 1001-1009, Sept. 2013.

[27] Thomas M. Cover and Joy A. Thomas, Elements of Information Theory, 2nd ed. New York, NY, USA: Wiley, 2006.

[28] Vyacheslav V. Prelov and Sergio Verdú, "Second-order asymptotics of mutual information," IEEE Trans. Inf. Theory, vol. 50, no. 8, pp. 1567-1580, Aug. 2004.

[29] Claude Berge, Topological Spaces: Including a Treatment of Multi-Valued Functions, Vector Spaces, and Convexity. Courier Corporation, 1997.

[30] Geoffrey C. Shephard, "Combinatorial properties of associated zonotopes," Canad. J. Math., vol. 26, no. 2, pp. 302-321, Feb. 1974.

[31] Ram Zamir and Meir Feder, "On the volume of the Minkowski sum of line sets and the Entropy-Power Inequality," IEEE Trans. Inf. Theory, vol. 44, no. 7, pp. 3039-3043, Nov. 1998.

[32] Stefan M. Moser, Information Theory (Lecture Notes), 6th ed. Signal and Inf. Proc. Lab., ETH Zürich, Switzerland, and Inst. Commun. Eng., Nat. Chiao Tung Univ., Hsinchu, Taiwan, 2018. Available: http://moser-isi.ethz.ch/scripts.html 